\title{
Physical properties and evolution of (Sub-)millimeter selected galaxies in the galaxy formation simulation SHARK
}

\author{
Claudia del P. Lagos ${ }^{1,2,3 \star}$, Elisabete da Cunha ${ }^{1,2}$, Aaron S. G. Robotham ${ }^{1,2}$ \\ Danail Obreschkow ${ }^{1,2}$, Francesco Valentino ${ }^{3,4}$, Seiji Fujimoto ${ }^{3,4}$, \\ Georgios E. Magdis ${ }^{3,4,5}$, Rodrigo Tobar ${ }^{1}$ \\ ${ }^{1}$ International Centre for Radio Astronomy Research (ICRAR), M468, University of Western Australia, 35 Stirling Hwy, Crawley, \\ WA 6009, Australia. \\ ${ }^{2}$ ARC Centre of Excellence for All Sky Astrophysics in 3 Dimensions (ASTRO 3D). \\ ${ }^{3}$ Cosmic Dawn Center (DAWN). \\ ${ }^{4}$ Niels Bohr Institute, University of Copenhagen, Lyngbyvej 2, DK-2100 Copenhagen, Denmark. \\ ${ }^{5}$ DTU-Space, Technical University of Denmark, Elektrovej 327, DK-2800 Kgs. Lyngby, Denmark.
}

Accepted XXX. Received YYY; in original form ZZZ

\begin{abstract}
We thoroughly explore the properties of (sub)-millimeter ( $\mathrm{mm}$ ) selected galaxies (SMGs) in the SHARK semi-analytic model of galaxy formation. Compared to observations, the predicted number counts at wavelengths $(\lambda) 0.6-2 \mathrm{~mm}$ and redshift distributions at $0.1-2 \mathrm{~mm}$, agree well. At the bright end ( $\gtrsim 1 \mathrm{mJy})$, SHARK galaxies are a mix of mergers and disk instabilities. These galaxies display a stacked FUV-toFIR spectrum that agrees well with observations. We predict that current optical/NIR surveys are deep enough to detect bright $(>1 \mathrm{mJy}) \lambda=0.85-2 \mathrm{~mm}$-selected galaxies at $z \lesssim 5$, but too shallow to detect counterparts at higher redshift. A James Webb Space Telescope 10,000s survey should detect all counterparts for galaxies with $S_{0.85 \mathrm{~mm}} \gtrsim 0.01 \mathrm{mJy}$. We predict SMG's disks contribute significantly (negligibly) to the rest-frame UV (IR). We investigate the $0 \leq z \leq 6$ evolution of the intrinsic properties of $>1 \mathrm{mJy} \lambda=0.85-2 \mathrm{~mm}$-selected galaxies finding their: (i) stellar masses are $>10^{10.2} \mathrm{M}_{\odot}$, with the $2 \mathrm{~mm}$ ones tracing the most massive galaxies $\left(>10^{11} \mathrm{M}_{\odot}\right)$; (ii) specific star formation rates (SFR) are mildly $(\approx 3-10 \times)$ above the main sequence (MS); (iii) host halo masses are $\gtrsim 10^{12.3} \mathrm{M}_{\odot}$, with $2 \mathrm{~mm}$ galaxies tracing the most massive halos (proto-clusters); (iv) SMGs have lower dust masses $\left(\approx 10^{8} \mathrm{M}_{\odot}\right)$, higher dust temperatures $(\approx 40-45 \mathrm{~K})$ and higher rest-frame V-band attenuation $(>1.5)$ than MS galaxies; (v) sizes decrease with redshift, from $4 \mathrm{kpc}$ at $z=1$ to $\lesssim 1 \mathrm{kpc}$ at $z=4$; (vi) the Carbon Monoxide line spectra of $S_{0.85 \mathrm{~mm}} \gtrsim 1$ mJy sources peak at $4 \rightarrow 3$. Finally, we study the contribution of SMGs to the molecular gas and cosmic SFR density at $0 \leq z \leq 10$, finding that $>1$ mJy sources make a negligible contribution at $z \gtrsim 3$ and $z \gtrsim 5$, respectively, suggesting current observations have unveiled the majority of the star formation at $0 \leq z \leq 10$.
\end{abstract}

Key words: galaxies: evolution - galaxies: formation - galaxies: ISM - submillimetre: galaxies

\section{INTRODUCTION}

One of the most intriguing galaxy populations observed to date are the so-called submillimeter galaxies (SMGs) - those that appear bright in the submillimeter (submm) or millimeter $(\mathrm{mm})$ bands (typically with fluxes $\gtrsim 1 \mathrm{mJy}$ ). They are rare in the local Universe, but are increasingly common

* E-mail: claudia.lagos@icrar.org with lookback time, with their number density peaking at around $z \approx 2$ (Chapman et al. 2005; Casey et al. 2014). Casey et al. (2012) showed that these galaxies make a negligible contribution to the total cosmic star formation rate density (CSFRD) locally, but make up $\gtrsim 50 \%$ of the CSFRD at $z \gtrsim 1$. Given their large contribution to the CSFRD in the early Universe and their number density, they are thought to be the progenitors of local massive elliptical galaxies (e.g. Cai et al. 2013; Toft et al. 2014; Valentino et al. 2020b). 
Hence, understanding the nature of SMGs and more generally, submm and mm-selected galaxies, is fundamental to unveiling the formation of galaxies at cosmic noon. Throughout this paper we will loosely refer to SMGs as galaxies that were selected from their submm or mm emission.

The advent of the Atacama Large Millimeter Array (ALMA) has opened an unprecedented window to study SMGs at high angular resolution and sensitivity across cosmic time. Karim et al. (2013), using ALMA, showed that the number counts of bright SMGs (those with fluxes at the observer-frame $870 \mu \mathrm{m} \gtrsim 1 \mathrm{mJy}$ ) obtained with previous single dish telescopes were highly affected by confusion, offering the first accurate measurements of the abundance of bright galaxies at $870 \mu \mathrm{m}$. ALMA has also allowed measurements of the number counts to go much deeper than ever before. Fujimoto et al. (2016); González-López et al. (2020) presented $1 \mathrm{~mm}$ number counts going as deep as $0.01 \mathrm{mJy}$ and $0.03 \mathrm{mJy}$, respectively, using ALMA with the former finding $\approx 2.5$ times more faint sources than the latter (see also Muñoz Arancibia et al. 2018). This difference is significant and implies that $S>0.01 \mathrm{mJy}$ sources recover the full far-infrared (FIR) background light or there is significant emission from fainter sources. It is however unclear whether these differences are solely due to cosmic variance or there are additional effects in play. The former is particularly significant given the pencil-beam surveys carried out so far with ALMA. ALMA has also allowed to remeasured the redshift distribution for these bright SMGs using both photometric and spectroscopic redshift determinations, showing that their abundance is highest at $z \approx 2-2.7$ (da Cunha et al. 2015; Dudzevičiūtè et al. 2020). Selecting on longer wavelengths generally leads to a higher redshift peak (Reuter et al. 2020). Despite this progress, most SMG redshifts come from their multi-wavelength photometry, which can suffer from significant systematic effects (Battisti et al. 2019; e.g. 44 out of 707 galaxies in Dudzevičiūtè et al. 2020 have confirmed redshifts). Spectroscopic redshift measurements require spectral scan techniques that can be time consuming but are nonetheless an important quest.

ALMA has also offered the opportunity to study the morphology of SMGs, overall finding that about half of the bright $\gtrsim 1 \mathrm{mJy} 870 \mu \mathrm{m}$ sources have disturbed morphologies (such as tidal tails or multiple nuclei), indicative of being galaxy merger-driven, while the other half are consistent with being disks (e.g. Cowie et al. 2018; Hodge et al. 2019; Gullberg et al. 2019). This is dramatically different to the local Universe, in which all infrared (IR)-bright galaxies have morphologies consistent with being ongoing galaxy mergers (Veilleux et al. 2002; da Cunha et al. 2010). This shows that these high-z SMGs are an inhomogeneous galaxy population. Besides their morphology, intrinsic properties, such as stellar masses and star formation rates (SFRs) have been derived, showing that bright SMGs are massive galaxies, $M_{\star} \approx 10^{10}-10^{11} \mathrm{M}_{\odot}$, with SFRs $\approx 200-300 \mathrm{M}_{\odot}$, on average (da Cunha et al. 2015; Dudzevičiūtè et al. 2020). This indicates that these SMGs are only modestly above the starforming main sequence (MS) in the SFR-stellar mass plane, by factors of $\approx 3-5$, on average. This again shows the different nature of these high-z SMGs compared to local ones, which are associated with extreme starbursts that are $\gtrsim 10$ times above the local Universe MS. This is not necessarily surprising, as the MS is strongly evolving with redshift, and hence high SFRs are more common in the early Universe (Elbaz et al. 2010; Whitaker et al. 2012). Interestingly, measurements of the sizes of these bright SMGs show that they are not so different from MS galaxies of the same stellar mass (Ikarashi et al. 2015; Barro et al. 2016; Simpson et al. 2015; Fujimoto et al. 2017; Puglisi et al. 2019).

Despite this tremendous progress there are still areas that require significant exploration. Casey et al. (2018b) highlighted the fact that the very limited survey areas that have been accessed with ALMA imply that we still do not have firm constraints on the contribution of SMGs to the CSFRD of the Universe at $z \gtrsim 4$. Casey et al. (2018b) showed that in some extreme models of dusty galaxies this contribution may be several times higher than that measured from rest-frame ultraviolet (UV)-selected galaxies (Bouwens et al. 2012). When studying the multi-wavelength properties of SMGs, it is also clear that a significant fraction do not have optical-to-near IR (NIR) counterparts. Dudzevičiūtè et al. (2020) found that $10 \%$ of their 707 SMGs do not have detections in the IR Array Camera (IRAC) on the Spitzer Space Telescope, which either imply much higher redshifts or extreme obscuration. The upcoming James Webb Space Telescope (JWST) promises to change the landscape again, providing detections for most if not all observed SMGs to date as we show in this paper.

In galaxy formation simulations, the abundance and properties of SMGs have been notoriously difficult to reproduce (Casey et al. 2014). This has been investigated using both semi-analytic models and hydrodynamical simulations of galaxy formation. The former have the advantage of accessing much larger cosmological volumes allowing for robust statistical studies of the abundance of SMGs at different redshifts, at the expense of the detailed internal structure predictions of galaxies. On the other hand, the advantage of hydrodynamical simulations resides in predicting the internal structure of gas in galaxies, from which one can link to the morphologies being studied with ALMA, at the expense of sample size. These two techniques are therefore highly complementary. Regarding SMGs, Baugh et al. (2005), using the GALFORM semi-analytic model of galaxy formation, showed that assuming a universal initial stellar mass function (IMF) of stars made the model incapable of reproducing their number counts and redshift distribution, suggesting that a plausible solution was to allow for a top-heavy IMF during starbursts. This was latter confirmed by Lacey et al. (2016) with updated versions of GALFORM. Hayward et al. (2011, 2013) combined 34 idealised isolated galaxies and galaxy mergers hydrodynamical simulations with various empirical cosmological relations to get a statistical estimate of number counts and redshift distributions. They argued that variations to the IMF were not necessary to reproduce these observations; however, Cowley et al. (2019) showed that the assumptions for how to populate large galaxy samples with $850 \mu$ m proposed by Hayward et al. (2011) were inconsistent with the self-consistent predictions of GALFORM obtained using the radiative transfer (RT) code GRASIL (Granato et al. 2000). The latter predicted $\lesssim 10$ times less bright SMGs than the ones one would derived from applying the Hayward et al. (2011) prescriptions, showing that self-consistent predictions cannot be replaced by semi-empirical relations.

Significant progress has been recently reported in the 
literature in this area. Lagos et al. (2019), using the SHARK semi-analytic model of galaxy formation (Lagos et al. 2018) in combination with the spectral energy distribution (SED) code ProSpect (Robotham et al. 2020) and the RT analysis of the EAGLE hydrodynamical simulations of Trayford et al. (2020) showed for the first time that a cosmological galaxy formation simulation was capable of reproducing the far UV-to far IR emission of galaxies in a wide redshift range, including the SMG number counts and redshift distributions, in a self-consistent way using a universal IMF. The key to the success of SHARK was attributed to the adoption of attenuation curves that scale with the dust surface density of galaxies as reported by Trayford et al. (2020), which take into account the 3D distribution of star formation and dust in galaxies. Lovell et al. (2020) using the SimBa hydrodynamical simulations (Davé et al. 2019) coupled with the RT code Powerday (Narayanan et al. 2020) also found that their model was able to reproduce the SMG number counts and redshift distributions using a universal IMF. The fact that two independent galaxy formation simulations reached the same conclusions, robustly shows that a variable IMF is not required to reproduce these key observations of SMGs.

In the area of SMG intrinsic properties, McAlpine et al. (2019) presented a thorough study of SMGs at $1<z<3$ with $850 \mu \mathrm{m}$ fluxes $>1 \mathrm{mJy}$ in the EAGLE simulations (Schaye et al. 2015; Crain et al. 2015). They found these high-z SMGs are massive, $M_{\star} \approx 10^{11} \mathrm{M}_{\odot}$, and have SFR $\approx$ $100 \mathrm{M}_{\odot} \mathrm{yr}^{-1}$, in broad agreement with observations. They also find that the incidence of galaxy mergers in bright SMGs is very similar to the underlying galaxy population, suggesting that mergers are not the sole driver of SMGs in EAGLE, again in broad agreement with the observational inferences from the morphologies of SMGs. An important drawback of this investigation is that EAGLE underpredicts the abundance of bright SMGs (Cowley et al. 2019) and predicts a significant number of those to be at $z<1^{1}$, in tension with the observations. Ideally, one would like to explore the intrinsic properties of SMGs in a galaxy formation simulation that broadly reproduces the basic observables of number counts and redshift distributions. This is the focus of this work.

We use the SHARK semi-analytic model and the SED generation pipeline presented in Lagos et al. (2019) to study the FUV-to-MIR and carbon monoxide $(\mathrm{CO})$ emission and intrinsic properties of submm and mm-selected galaxies across cosmic time, $0 \leq z \leq 10$. The starting point is the fact that SHARK reproduces the $850 \mu \mathrm{m}$ number counts and redshift distributions, but here we extend the testing of SHARK to the full FIR spectrum. This paper is organised as follows: $\S 2$ presents a description of SHARK, the adopted $N$-body simulations and a summary of how several baryonic physical processes are modelled. $\S 2$ also presents a summary of how SEDs and CO spectral line energy distributions (SLEDs) are generated. $\S 3$ compares our predictions with observations of number counts at wavelengths from $650 \mu \mathrm{m}$ to $2 \mathrm{~mm}$, and redshift distributions of galaxies selected at wavelengths from $100 \mu \mathrm{m}$ to $2 \mathrm{~mm}$. $\S 4$ and $\S 5$ present the predicted UV-to-

\footnotetext{
1 The redshift distribution for EAGLE was obtained by selecting galaxies from their public database (McAlpine et al. 2015; Camps et al. 2018) with $S_{850 \mu \mathrm{m}}>1 \mathrm{mJy}$ (not shown here), which shows a peak of sources towards $z=0$.
}

MIR emission and intrinsic properties of SMGs, respectively, in SHARK and compares to observations where possible. $\S 6$ presents an analysis of the contribution of submm and $\mathrm{mm}-$ selected galaxies to the CSFRD and the cosmic molecular gas density at $0 \leq z \leq 10$, tackling the question of whether we are missing a significant SFR and cold gas source at high redshifts. Finally, $\S 7$ presents our conclusions.

\section{THE SHARK SEMI-ANALYTIC MODEL}

SHARK is an open source, flexible and highly modular $\mathrm{SAM}^{2}$, introduced in Lagos et al. (2018). The model runs over merger trees and halo/subhalo populations computed from the SURFs N-body simulations. Below we provide a summary of the SURFs suite in $\S 2.1$, then describe the physics included in SHARK in $\S 2.2$, and finally the way we compute SEDs and CO SLEDs for our simulated galaxies in $\S 2.3$ and $\S 2.4$, respectively.

\subsection{The SURFS $N$-body suite}

SHARK uses the SURFS suite of N-body, dark-matter (DM) only simulations (Elahi et al. 2018b), most of which have cubic volumes of $210 \mathrm{cMpc} / \mathrm{h}$ on a side, and span a range in particle number, currently up to 8.5 billion particles using a $\Lambda$ CDM Planck Collaboration et al. (2016) cosmology. These correspond to a total matter, baryon and $\Lambda$ densities of $\Omega_{\mathrm{m}}=0.3121, \Omega_{\mathrm{b}}=0.0491$ and $\Omega_{\mathrm{L}}=0.6879$, respectively, with a Hubble parameter of $H_{0}=h 100 \mathrm{Mpc}^{-1} \mathrm{~km} \mathrm{~s}^{-1}$ with $h=0.6751$, scalar spectral index of $n_{\mathrm{s}}=0.9653$ and a power spectrum normalization of $\sigma_{8}=0.8150$. All simulations were run with a memory lean version of the GADGET 2 code on the Magnus supercomputer at the Pawsey Supercomputing Centre. In this paper, we use the L210N1536 simulation, which has a cosmological volume of $(210 \mathrm{cMpc} / \mathrm{h})^{3}, 1536^{3} \mathrm{DM}$ particles with a mass of $2.21 \times 10^{8} \mathrm{~h}^{-1} \mathrm{M}_{\odot}$ and a softening length of $4.5 \mathrm{~h}^{-1}$ ckpc. Here, cMpc and ckpc denote comoving Mpc and kpc, respectively. SURFS produces 200 snapshots for each simulation at $z=0-24$, typically having a time span between snapshots in the range of $\approx 6-80 \mathrm{Myr}$. So far suRFS consists of 7 simulations, but this is an ever growing suite.

Merger trees and halo catalogs, which are the basis for SHARK (and generally any SAM), were constructed using the phase-space finder VELOCIRAPTOR ${ }^{3}$ (Elahi et al. 2019a; Cañas et al. 2019) and the halo merger tree code TreeFroG ${ }^{4}$, developed to work on VELOCIraptor (Elahi et al. 2019b). Poulton et al. (2018, 2019) show that TreeFrog+VELOCIRAPTOR lead to very well behaved merger trees, with orbits that are well reconstructed. Elahi et al. (2018a) also show that these orbits reproduce the velocity dispersion vs. halo mass inferred in observations. Cañas et al. (2019) show that the same code can be applied to hydrodynamical simulations to identify galaxies and that the performance of VELOCIRAPTOR is superior to spacefinders, even in complex merger cases. We refer to Lagos et al. (2018) for more details on how the merger trees and

\footnotetext{
2 https://github.com/ICRAR/shark

3 https://github.com/icrar/VELOCIraptor-STF/

4 https://github.com/pelahi/TreeFrog
} 
halo catalogs are constructed for SHARK, and to Elahi et al. (2019a,b); Cañas et al. (2019); Poulton et al. (2018) for more details on the VELOCIRAPTOR and TREEFrog software.

\subsection{Baryon physics in SHARK}

SHARK includes a large range of physical processes that are key in shaping galaxy formation and evolution. These are (i) the collapse and merging of DM halos; (ii) the accretion of gas onto halos, which is modulated by the DM accretion rate; (iii) the shock heating and radiative cooling of gas inside DM halos, leading to the formation of galactic disks via conservation of specific angular momentum of the cooling gas; (iv) star formation (SF) in galaxy disks; (v) stellar feedback from the evolving stellar populations; (vi) chemical enrichment of stars and gas; (vii) the growth via gas accretion and merging of supermassive black holes; (viii) heating by AGN; (ix) photoionization of the intergalactic medium; (x) galaxy mergers driven by dynamical friction within common DM halos which can trigger starbursts and the formation and/or growth of spheroids; (xi) collapse of globally unstable disks that also lead to starbursts and the formation and/or growth of bulges (we refer to this mechanism as "disk instabilities"). In SHARK, galaxy mergers are considered to be of any mass ratio, as long as two galaxies are involved in the collision. Disk instabilities on the other hand, are a secular process, which happens in galaxies whose disks are globally unstable. In this case, the disk is instantaneously collapsed into a central overdensity (i.e. forming a bulge or contributing to it), and the inflowing gas triggers a starburst. SHARK adopts a universal Chabrier (2003) initial mass function (IMF). Lagos et al. (2018) include several different models for gas cooling, AGN, stellar and photoionisation feedback, star formation and dynamical friction timescales. Here, we adopt the default SHARK model (see models and parameters adopted in Lagos et al. 2018; their Table 2).

In SHARK, SF is computed from the surface density of molecular gas. SF in galaxy disks follow $\Sigma_{\mathrm{SFR}}=\Sigma_{\mathrm{mol}} / \tau_{\mathrm{mol}}$, where $\Sigma_{\text {SFR }}$ and $\Sigma_{\text {mol }}$ are the SFR and molecular gas surface densities, respectively, while $\tau_{\mathrm{mol}}=1 \mathrm{Gyr}$ is the molecular gas depletion timescale. The adopted value comes from observational constraints (Leroy et al. 2008; Bigiel et al. 2010) and is assumed to be constant across cosmic time. The latter is motivated by the Carbon Monoxide (CO) observations of galaxies of Genzel et al. (2015) and Schinnerer et al. (2016) at $0 \lesssim z \lesssim 4$, which show little evolution of $\tau_{\mathrm{mol}}$. In the case of starbursts triggered by galaxy mergers and disk instabilites, the SF model used resembles that for SF in disks but with a shorted depletion timescale, $\Sigma_{\mathrm{SFR}}=\Sigma_{\text {mol }} /\left(\tau_{\text {mol }} / f_{\text {boost }}\right)$, with $f_{\text {boost }}=10$. The latter is motivated by observational constraints (Sargent et al. 2014). We will refer to these two modes of SF as "normal" (SF in disks) and "burst" (SF triggered by galaxy mergers and global disk instabilities) modes. The latter is the one that builds bulges in SHARK. Although the transfer of gas during galaxy mergers and disk instabilities to the bulge is instantaneous, the SF episode is slow given the SF law we adopt, which means that during starbursts the molecular gas is consumed typically in a few 100 Myr.

We numerically solve the differential equations (DEs) of mass, metals and angular momentum exchange between the different baryon reservoirs (see Eqs. 49-64 in Lagos et al. 2018), only setting an accuracy to which these equations are solved. The baryon reservoirs in the model are: gas outside halos, hot and cold gas inside halos but outside galaxies, ionised/atomic/molecular gas and stars in disks and bulges in galaxies, and super-massive black holes. Together with intrinsic properties of galaxies at the output times, we also store the star formation history ( $\mathrm{SFH})$ and metallicity history $(\mathrm{ZFH})$ of the stars that form at each timestep prior to the output time. These are later used to compute each galaxy's SED. The solving of the set of DEs as well as the storing of SFH and ZFH is done for all galaxy stellar components separately: disks and bulges. In addition, we track the two growth mechanisms of the latter, disk instabilities and galaxy mergers. Note that at $z \gtrsim 1$, these bulges are very active and harbour high surface densities of SFR (see Lagos et al. 2019 for examples of the SEDs of passive and active bulges in SHARK). This tracking of stellar component allows us to build independent SEDs for galaxy disks, bulge stars that formed via galaxy mergers, and bulge stars that formed via disk instabilities. This is important to understand which galaxy components and types of bulges dominate over different luminosities and cosmic times.

The model parameters of the default SHARK model were tuned to the $z=0,1,2$ stellar mass functions (SMFs; Wright et al. 2018), the $z=0$ black hole-bulge mass relation (McConnell \& Ma 2013) and mass-size relations (Lange et al. 2016). The model also reproduces very well observational results that are independent from those used for the tuning, such as the total neutral, atomic and molecular hydrogenstellar mass scaling relations at $\mathrm{z}=0$, the CSFRD density evolution at $z \approx 0-4$, the cosmic density evolution of the atomic and molecular hydrogen at $z \lesssim 2$ or higher in the case of the latter, the mass-metallicity relations for the gas and stars, the contribution to the stellar mass by bulges and the SFR-stellar mass relation in the local Universe (see Lagos et al. 2018 for more details). Davies et al. (2019) also show that SHARK reproduces the scatter around the MS of star formation in the SFR-stellar mass plane; Chauhan et al. $(2019,2020)$ show that SHARK reproduces very well the HI mass and velocity width of galaxies and the HI-halo mass relation observed in the ALFALFA survey; Amarantidis et al. (2019) show that the AGN LFs agree well with observations in the X-rays and radio wavelengths; and Bravo et al. (2020) show that optical colour distributions and passive fractions of SHARK galaxies agree reasonably well with GAMA observations. These can be seen as true successes of the model as none of these observations were used in the processes of tuning the free parameters.

\subsection{Spectral Energy Distributions of galaxies in SHARK}

The way we compute SEDs for galaxies is thoroughly described in Lagos et al. (2019); here we provide a brief overview.

We make use of two packages: ProSPECT ${ }^{5}$ and VIPER-

5 https://github.com/asgr/ProSpect and for an interactive ProSpect web tool see http://prospect.icrar.org/, which is recommended as an education tool. 

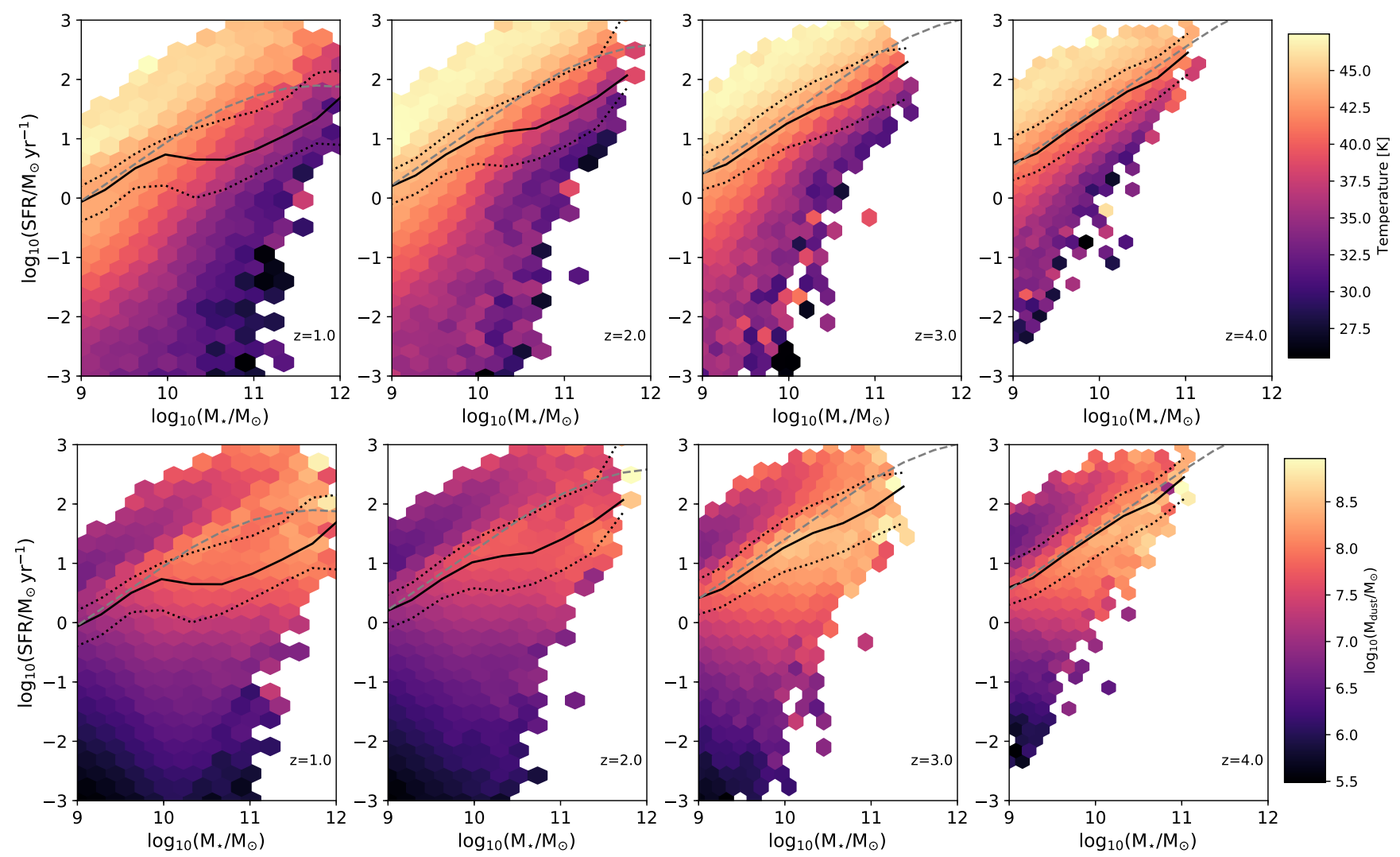

Figure 1. Top panels: SFR vs. stellar masses plane at four different redshifts from $z=1$ to $z=4$, as labelled, using a $\Delta z=0.15$. Pixels with $\geq 10$ galaxies are coloured by their median dust temperature. Solid and dotted lines show the median SFR and $16^{\text {th }}-84^{\text {th }}$ percentile ranges in bins of stellar mass, respectively, of all galaxies with SFR $>0$. This can be considered as the MS in SHARK, except at the high-mass end $\left(\gtrsim 10^{10.2} \mathrm{M}_{\odot}\right)$, where galaxies are affected by AGN feedback at $z \lesssim 3$. For reference, we also show the MS inferred observationally by Schreiber et al. (2015) as dashed lines. Galaxies above the MS are on average hotter than those on or below. Massive MS galaxies are also colder than low mass MS galaxies. Bottom panels: As in the top panels but colouring by the median dust mass, as indicated by the colour bar. Dust mass is maximal at the MS, decreasing when going above and below, at fixed stellar mass.

FISH $^{6}$. ProSpect (Robotham et al. 2020) combines the GALEXev stellar synthesis libraries Bruzual \& Charlot (2003) (BC03 from hereafter) and/or EMILES (Vazdekis et al. 2016) with the two-component dust attenuation model of (Charlot \& Fall 2000) and dust re-emission using the templates of (Dale et al. 2014). The latter cover up to a restframe wavelength of $1,000 \mu \mathrm{m}$. On top of ProSpeCt sits VIPERFISH, which allows for simple use of SHARK SFHs and ZFHs, and generation of the desired SED through target filters. For this paper we include a large range of bands: GALEX FUV and NUV, SDSS u, g, r, i, z VISTA Y, J, H, K, WISE bands 1, 2, 3, 4, Spitzer IRAC bands 1, 2, 3, 4, Herschel PACS $70 \mu \mathrm{m}, 100 \mu \mathrm{m}, 160 \mu \mathrm{m}$, and SPIRE $250 \mu \mathrm{m}, 350 \mu \mathrm{m}, 500 \mu \mathrm{m}$, JCMT $450 \mu \mathrm{m}$ and $850 \mu \mathrm{m}$, and ALMA bands $9,8,7,6,5$ and 4 . For all, except the ALMA bands, we use the published filter responses that come with ProSpect, while for ALMA we use a top-hat filter over the frequency range of each band ${ }^{7}$. This is done to thoroughly study the UV-to-IR emission of submm and mm-selected galaxies. When using a lightcone, galaxy SEDs are shifted

\footnotetext{
6 https://github.com/asgr/Viperfish

7 The frequency range adopted can be found here https://www . eso.org/public/teles-instr/alma/receiver-bands/.
}

to the observer-frame according to their redshifts. Note that due to the wavelength limit of the IR templates of Dale et al. (2014), observer-frame galaxy emission in ALMA bands 5 and 4 is only computed for galaxies at $z \geq 0.42$ and $z \geq 0.84$, respectively. We are currently working on PROSPECT to include a module for radio continuum emission from both freefree and synchrotron processes, which will naturally allow our machinery to extend our predictions to band 3 and the Square Kilometer Array frequency coverage.

In VIPERFISH, we attenuate and re-emit the light due to birth clouds first, and then attenuate and re-emit the light due to the diffuse ISM. The Charlot \& Fall (2000) (hereafter CF00) absorption curve for stars in the diffuse ISM and birth clouds can be written as follows

$$
\begin{aligned}
\tau_{\mathrm{ISM}} & =\hat{\tau}_{\mathrm{ISM}}(\lambda / 5500 \AA)^{\eta_{\mathrm{ISM}}}, \\
\tau_{\mathrm{BC}} & =\hat{\tau}_{\mathrm{BC}}(\lambda / 5500 \AA)^{\eta_{\mathrm{BC}}},
\end{aligned}
$$

respectively, where $\hat{\tau}_{\mathrm{ISM}}$ and $\hat{\tau}_{\mathrm{BC}}$ are the optical depth at $5500 \AA$ in the diffuse ISM and birth clouds, respectively, and $\eta_{\text {ISM }}$ and $\eta_{\mathrm{BC}}$ are the power-law indices that control the dependence on wavelength for the diffuse ISM and birth clouds, respectively. In SHARK we scale the CF00 parameters above depending on local properties of galaxies. 


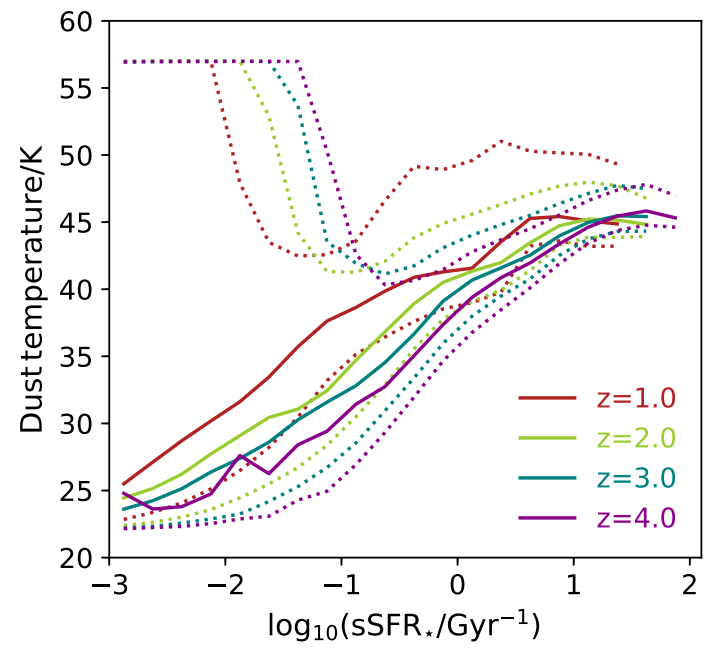

Figure 2. Dust temperature vs. specific SFR at four different redshifts from $z=1$ to $z=4$, as labelled, using a $\Delta z=$ 0.15 . Solid and dotted lines show the medians and $16^{\text {th }}-84^{\text {th }}$ percentile ranges in bins of sSFR, respectively. Note that at $\log _{10}\left(\mathrm{sSFR} / \mathrm{Gyr}^{-1}\right) \lesssim-1$, the $84^{\text {th }}$ percentile saturates at a temperature of $\approx 57 \mathrm{~K}$. This is due to galaxies having their IR emission being almost entirely dominated by birth cloud emission.

In the case of birth clouds, we compute the optical depth from the gas metallicity and a typical cloud surface density (see Eq. 6 in Lagos et al. 2019). The latter is $\Sigma_{\text {gas,cl }}=\max \left[\Sigma_{\mathrm{MW}, \mathrm{cl}}, \Sigma_{\text {gas }}\right]$, with $\Sigma_{\mathrm{MW}, \mathrm{cl}}=85 \mathrm{M}_{\odot} \mathrm{pc}^{-2}$ (Krumholz et al. 2009), $\Sigma_{\text {gas }}$ being the diffuse medium gas surface density, $0.5 \Sigma_{\text {gas }} / \pi r_{50}^{2}$, and $r_{50}$ being the half-gas mass radius. The wavelength power-law index is fixed to $\eta_{\mathrm{BC}}=-0.7$.

For the diffuse dust we use the scaling derived from the RT analysis of EAGLE galaxies at $0 \leq z \leq 2$ using SKIRT (Camps \& Baes 2015) proposed by Trayford et al. (2020). These consist of $\hat{\tau}_{\text {ISM }}$ and $\eta_{\text {ISM }}$ varying as a function of the dust surface density, $\Sigma_{\text {dust }}$, which are redshift independent. We compute $\Sigma_{\text {dust }}$ independently for the disk and the bulges of galaxies. To compute $\Sigma_{\text {dust }}$, we include the effect of inclination as described in Lagos et al. (2019) (Eq. 2).

Dust masses in SHARK are calculated for each galaxy component from their gas mass and metallicity, following the best fit relation between the dust mass and the latter two galaxy properties in the local Universe of Rémy-Ruyer et al. (2014) (see Fig. 1 in Lagos et al. 2019). The surface density is then computed from the half-gas mass radius of the disk or bulge and the inclination in which the galaxy is seen - it therefore depends on where the observer is located. The details of these calculations can be found in $\S 3.1$ of Lagos et al. (2019).

For the remission of the absorbed light in the IR, we use the Dale et al. (2014) templates, which are parametrised by a power-law index that controls the global dust emission depending on the local interstellar radiation field $\mathrm{U}$, with a fraction $\mathrm{d} M_{\text {dust }}$ of dust mass being heated by $U^{-\alpha_{\mathrm{SF}}} \mathrm{d} U$, and $U=1$ being the local interstellar radiation field of the solar neighbourhood. In VIPERFISH, we adopt $\alpha_{\mathrm{SF}}=3$ for the diffuse ISM and $\alpha_{\mathrm{SF}}=1$ for the birth clouds, motivated by the $\alpha_{\mathrm{SF}}$ values derived in Dale et al. (2014) for a range of galaxies going from normal star-forming (typically $\alpha_{\mathrm{SF}} \approx$ $2-3$ ) to highly starbursting (typically $\alpha_{\mathrm{SF}} \approx 1-2$ ). The SED model adopted here is referred to as "EAGLE- $\tau$ RR14" in Lagos et al. (2019).

From the re-emission of the absorbed light in the IR we can compute an effective dust temperature. We use the total IR luminosity emitted by the screen and birth cloud components of the IR templates of Dale et al. (2014). The values adopted here for $\alpha_{\mathrm{SF}}$ for the screen and birth cloud components roughly correspond to effective dust temperatures of $22 \mathrm{~K}$ and $57 \mathrm{~K}$, respectively, which we referred to as $T_{\text {diff }}$ and $T_{\mathrm{bc}}$, respectively. This way, a single temperature is computed following da Cunha et al. (2015):

$T_{\mathrm{eff}}=\frac{\left(L_{\mathrm{IR}, \mathrm{diff}} T \operatorname{diff}+L_{\mathrm{IR}, \mathrm{bc}} T_{\mathrm{bc}}\right)}{L_{\mathrm{IR}}}$,

where $T_{\text {eff }}$ is the effective dust temperature, $L_{\mathrm{IR}}=L_{\mathrm{IR}, \mathrm{diff}}+$ $L_{\mathrm{IR}, \mathrm{bc}}$, and $L_{\mathrm{IR} \text {,diff }}$ and $L_{\mathrm{IR}, \mathrm{bc}}$ are the IR luminosity produced by the diffuse and birth cloud dust components, respectively. The latter is approximately equivalent to the IR emission in the $4-1000 \mu \mathrm{m}$ wavelength range.

The most simplistic assumption we make in the build-up of SEDs of SHARK galaxies, are the fixed $\alpha_{\mathrm{SF}}$ for birth clouds and diffuse dust, which has consequences on the range of dust temperature SHARK galaxies can have. The top panels of Fig. 1 show the median dust temperature of galaxies in the SFR-stellar mass plane at four different redshifts, from $z=1 \pm 0.15$ to $z=4 \pm 0.14$. We show the median SFR of all galaxies with a SFR $>0$ and the $1 \sigma$ scatter around the median for reference. We consider this to be a good proxy for the MS position in SHARK, except in massive galaxies, $M_{\star} \gtrsim$ $10^{10.2} \mathrm{M}_{\odot}$, at $z \lesssim 3$, which are affected by AGN feedback. The latter becomes more significant towards low redshift. For reference, we show the observational inferences of the MS from Schreiber et al. (2015), which confirm that deviations in SHARK take place in massive galaxies.

Lines of constant dust temperature become shallower with increasing redshift on the MS and below. Above the MS (i.e. above the $84^{\text {th }}$ percentile range), lines of constant dust temperature run parallel to the MS with a weak trend of increasing temperature with increasing SFR at fixed stellar mass. If we focus on the MS and below, at $z=1 \pm 0.15$ and $z=2 \pm 0.15$, the lines of constant dust temperature being steeper than the MS lead to the trend of low-mass galaxies having on average hotter dust than massive galaxies; by $z=4 \pm 0.15$ lines of constant temperature are parallel to the SFR MS even in the MS and below. The trends above largely mimic the variations in the SFR surface density and specific SFR of galaxies in the SFR-stellar mass plane. Fig. 2 shows the dependence of the dust temperature on specific SFR (sSFR). Dust temperature systematically increases with increasing sSFR, which is qualitatively similar to what da Cunha et al. (2008) find for MAGPHYS (see their Fig. 14). There is a mild redshift evolution, in a way that at fixed sSFR, the high-z galaxies tend to have lower $T_{\text {dust }}$. The distribution of dust temperature at sSFR $<0.05 \mathrm{Gyr}^{-1}$ is highly bimodal with the FIR emission being either fully dominated by birth cloud emission or diffuse dust. The reason for this is that the SFR in these galaxies is highly stochastic. Small episodes of SF lead to FIR emission being fully dominated by birth cloud emission. However, the 
mode in the low sSFR regime is dominated by diffuse dust emission only. Schreiber et al. (2018) measured $T_{\text {dust }}$ of MS galaxies at $0.5<z<4$ assuming a simple Wien's law, and they find that at $z \leq 1$ massive galaxies $\left(>10^{11} \mathrm{M}_{\odot}\right)$ have lower $T_{\text {dust }}$ than lower mass galaxies, in qualitative agreement with our predictions. For main sequence galaxies of $M_{\star}>10^{11} \mathrm{M}_{\odot}$ they found $T_{\text {dust }}$ to increase from $25 \mathrm{~K}$ at $z=1$ to $40 \mathrm{~K}$ at $z=4$ (see also Cowley et al. 2017a). In ShARK, for those galaxies $T_{\text {dust }}$ increases from $\approx 30 \mathrm{~K}$ at $z=1$ to $\approx 42 \mathrm{~K}$ at $z=4$ in broad agreement with the observations within the systematic uncertainties.

Because the FIR emission is regulated by the dust temperature and mass, it is important to also investigate the evolution of dust masses in SHARK galaxies. The bottom panels of Fig. 1 show the median dust mass of galaxies in the SFR-stellar mass plane at $z \approx 1-4$. The dust mass is maximal in the MS, and decreases towards higher and lower SFRs at fixed stellar mass. This is a natural outcome of the star formation model adopted in SHARK. Galaxies that undergo starbursts (those triggered by galaxy mergers and global disk instabilities) have a molecular gas depletion timescale 10 times shorter than galaxies that undergo normal star formation in their disks. This means that for the same amount of molecular gas, the SFR is 10 times higher in a starburst galaxy. This leads to galaxies above the MS (which are mostly in the starburst mode) having lower dust masses (due to the lower gas content) than MS galaxies at fixed stellar mass at all redshifts. Below the MS the trend is driven by the underlying lower molecular gas masses that lead to both lower SFRs and dust masses. At fixed distance to the MS, the dust mass increases with increasing stellar mass.

\subsection{Carbon Monoxide emission of galaxies}

We compute the CO SLED from the $1 \rightarrow 0$ to the $10 \rightarrow 9$ rotational transitions, using the Photon-dominated regions (PDR) modelling introduced in Lagos et al. (2012). In short, Lagos et al. (2012) used a large grid of PDR models from Bayet et al. (2011), which were run using fiducial Giant Molecular Cloud (GMC) properties. These GMC models adopted a gas density of $\mathrm{n}_{\mathrm{H}}=10^{4} \mathrm{~cm}^{-4}$ and an attenuation of $A_{\mathrm{V}}=3 \mathrm{mag}$, and were run for $10^{6} \mathrm{yr}$. These PDR models were run for a range of interstellar radiation fields, $G_{\mathrm{UV}}$, gas metallicities, $Z_{\text {gas }}$ and $X$-ray fluxes, $F_{\mathrm{X}}$. In Bayet et al. (2011) the latter are modelled as cosmic ray dominated regions (CRDRs), which are found to behave very similarly to X-ray dominated regions (and hence can be used in a similar fashion). The output of an individual run (which is then the combined effect of the PDR+CRDR) is 10 molecular hydrogen-to-CO conversion factors, from $1 \rightarrow 0$ to the $10 \rightarrow 9$ (see Table 1 in Lagos et al. 2012).

To compute a CO Spectra Line Energy Distribution (CO SLED) for each individual SHARK galaxy, we first compute $G_{\mathrm{UV}}, Z_{\text {gas }}$ and $F_{\mathrm{X}}$, and interpolate over the grid of PDR models (with all properties in $\log _{10}$ ) using the PYTHON tool interPolate.LinEARNDINTERPOLATOR of the SciPy package (Virtanen et al. 2020). We do this for galaxy disks and bulges separately, and sum their contributions to derive a total CO SLED. Below we describe our calculation of $G_{\mathrm{UV}}$ and $F_{\mathrm{X}}$.

The interstellar radiation field relative to the solar neighbourhood value, $G_{0}=1.6 \times 10^{-3} \mathrm{erg} \mathrm{cm}^{-2} \mathrm{~s}^{-1}$, is computed as

$\frac{G_{\mathrm{UV}}}{G_{0}}=\frac{\Sigma_{\mathrm{SFR}} / \Sigma_{\mathrm{SFR}}^{0}}{\left(Z_{\text {gas }} / Z_{\odot}\right)\left(\Sigma_{\text {gas }} / \Sigma_{\text {gas }}^{0}\right)}$,

where $\Sigma_{\text {SFR }}$ and $\Sigma_{\text {gas }}$ are the SFR and gas, respectively, surface density computed at the disk and bulge half-stellar radius. $\Sigma_{\mathrm{SFR}}^{0}=10^{-3} \mathrm{M}_{\odot} \mathrm{yr}^{-1} \mathrm{kpc}^{-2}$ and $\Sigma_{\text {gas }}^{0}=10 \mathrm{M}_{\odot} \mathrm{pc}^{-2}$ are the solar neighbourhood SFR (Bonatto \& Bica 2011) and gas mass (Chang et al. 2002) surface densities. The physical interpretation of the numerator of Eq. 4 is that the local UV radiation field should be proportional to the SFR surface density as young stars provide the bulk of the UV emission, while the denominator accounts for attenuation. In a slab, the transmission probability of UV photons, $\beta_{\mathrm{UV}}$, scales with the optical depth, so that $\beta_{\mathrm{UV}} \sim\left(1-e^{-\tau_{\mathrm{UV}}}\right) / \tau_{\mathrm{UV}}$. The optical depth, on the other hand, depends on the gas metallicity and column density of atoms as $\tau_{\mathrm{UV}} \propto Z_{\text {gas }} N_{\mathrm{H}}$. In optically thick gas $\left(\tau_{\mathrm{UV}} \gg 1\right), \beta_{\mathrm{UV}} \sim \tau_{\mathrm{UV}}^{-1}$.

The X-ray radiation field, $F_{\mathrm{X}}$ is computed from the hard $(2-10 \mathrm{kEV}) \mathrm{X}$-ray luminosity of AGNs, $L_{\mathrm{X}}$, computed as in Amarantidis et al. (2019) (see their Eqs. 1-3) for SHARK, divided by the surface of the sphere of radius equivalent to the disk or bulge half-stellar mass radius, $4 \pi r_{50}^{2}$. This assumes that hard X-rays photons are not absorbed.

Some important caveats in the CO SLED modelling above are worth discussing. We assume galaxy inclination can be ignored for simplicity, as it allows us to use precomputed PDR models. This is likely not correct as clouds in a disk can lead to enhanced shielding between different clouds. This may be an important effect (especially at highz) given the high $\mathrm{CO}$ opacity of the main isotopes. Another important caveat is that GMCs that dominate star formation at $z \approx 2$ can be $\approx 100-1000$ times more massive than local GMCs (Swinbank et al. 2010). This may lead to increased self-shielding inside GMCs. Both cases (enhanced self-shielding or cloud-to-cloud shielding) could lead to the CO luminosity becoming fainter. Despite these caveats, we show that SHARK can broadly reproduce the observed CO SLEDs in normal star-forming galaxies at $z=1-3$ (Hamanowicz et al. in preparation) and SMGs $(\S 5.7)$.

Fig. A3 shows the median brightness $\mathrm{CO}(1-0)$ luminosity, $L_{\mathrm{CO}(1-0)}^{\prime}$ of galaxies in the SFR-stellar mass plane at $z=1-4$. $L_{\mathrm{CO}(1-0)}^{\prime}$ varies in complex ways in this plane: at fixed stellar mass $L_{\mathrm{CO}(1-0)}^{\prime}$ increases with increasing SFR up to the MS. Above the MS, $L_{\mathrm{CO}(1-0)}^{\prime}$ decreases for a bit, followed by an increase in a way that the most starbursting galaxies have the highest $L_{\mathrm{CO}(1-0)}^{\prime}$. This complex behaviour is driven by the combination of variations in the $\mathrm{H}_{2}$ mass and in the conversion factor from $\mathrm{H}_{2}$ to $\mathrm{CO}(1-0)$. Note that $L_{\mathrm{CO}(1-0)}^{\prime}$ varies in different ways than $M_{\text {dust }}$ in the SFRstellar mass plane in SHARK.

\section{SMG NUMBER COUNTS AND REDSHIFT DISTRIBUTIONS}

In order to select a population of SMGs in SHARK, we make use of the deep simulated lightcone introduced in Lagos et al. (2019). This corresponds to an area of $107 \mathrm{deg}^{2}$ including all galaxies with a dummy magnitude, computed 

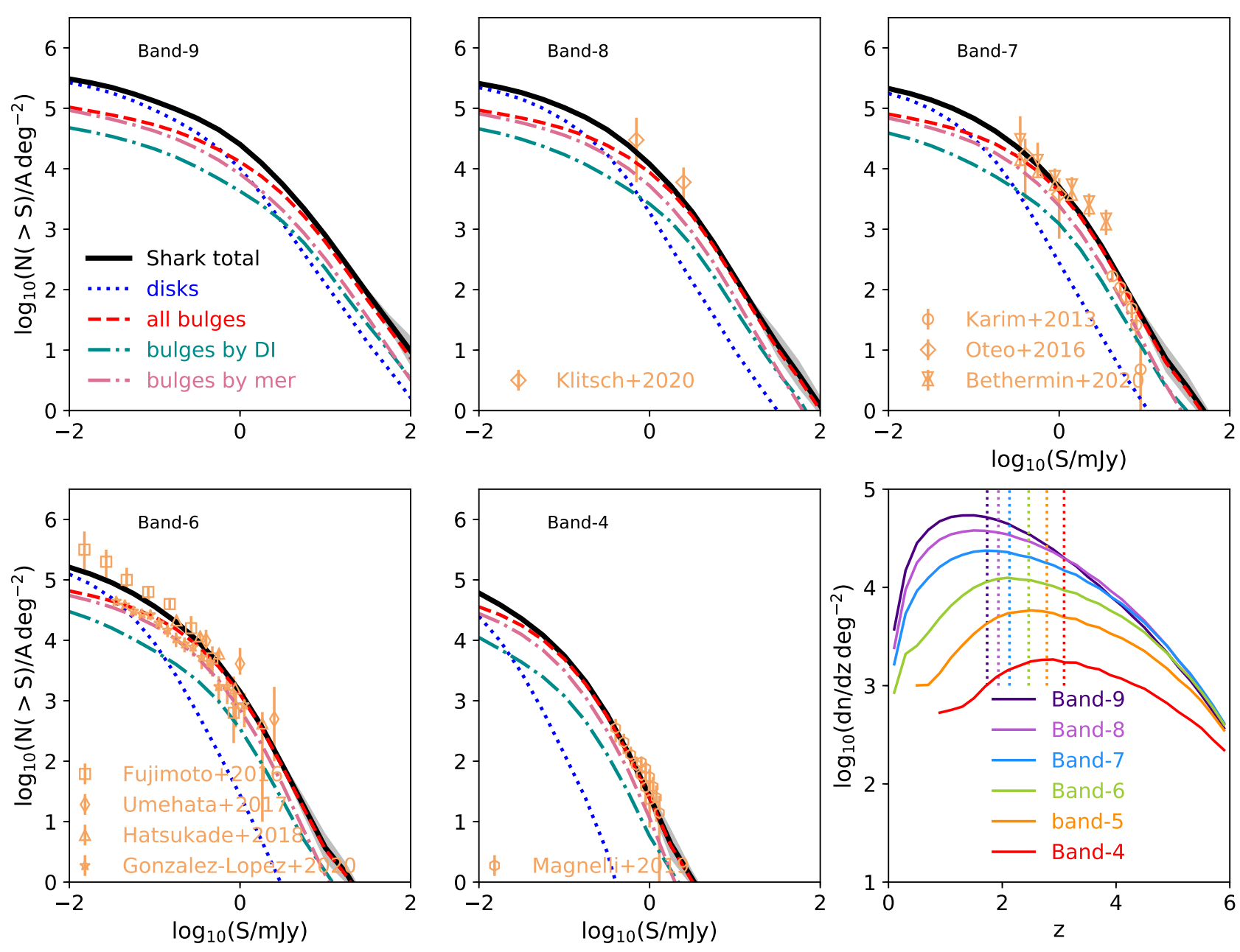

Figure 3. Number counts for our SHARK $107 \mathrm{deg}^{2}$ deep lightcone at the ALMA bands 9, 8, 7, 6 and 4, as labelled in each panel. The contribution from disks and bulges are shown as dotted and dashed lines. We split starbursts further into those driven by galaxy mergers and disk instabilities and show them as dot dashed lines as labelled. We compute a bootstrap error on the total number counts and present those as shaded grey region. Observations from Klitsch et al. (2020) for band-8, Karim et al. (2013), Oteo et al. (2016) and Bethermin et al. (2020) for band-7, Fujimoto et al. (2016), Umehata et al. (2017), Hatsukade et al. (2018), González-López et al. (2020) for band-6 and Magnelli et al. (2019) for band-4 are shown as symbols. For Bethermin et al. (2020) we show two measurements: all their detections (down-pointing triangles) and those considered secured (up-pointing triangles). The bottom-right panel shows the predicted redshift distribution of $>0.1$ mJy sources selected in ALMA bands 9 to 4 , as labelled. Vertical dotted lines show the respective median redshifts. SHARK shows that the longer the wavelength used for the selection, the higher the peak redshift of the galaxies.

assuming a stellar mass-to-light ratio of $1,<32$ and at $0 \leq z \leq 6$. Chauhan et al. (2019) provide a detailed description of the construction of lightcones using SHARK and StINGRAY. The latter is the lightcone software originally developed by Obreschkow et al. (2009) and further developed by Obreschkow et al. (in preparation ${ }^{8}$ ). The exact way the simulated box at different snapshots were tiled and the angular momentum vector of the galaxies determine the inclination in which galaxies are viewed by the observer. The latter are considered in the calculation of dust attenuation (§ 2.3).

We use this lightcone to first analyse the overall number

8 https://github.com/obreschkow/stingray/ counts and redshift distributions of galaxies selected using different IR bands and flux cuts ( $\$ 3)$. We then focus on the UV-to-MIR properties of ALMA-selected galaxies in SHARK $(\S 4)$. Finally, we focus on the intrinsic properties of SMGs and their environments throughout cosmic time ( $\S 5)$.

Lagos et al. (2019) showed that the predicted GALEX NUV to JCMT $850 \mu$ m number counts agreed well with observations. Here, we extend this testing of SHARK to a large range of ALMA bands, from band $9(\lambda=[0.4,0.5] \mathrm{mm})$ to band $4(\lambda=[1.8,2.4] \mathrm{mm})$. ALMA is the best instrument to compare our direct predictions of galaxy number counts with as its excellent angular resolution allows a robust identification of the emission of individual galaxies (Karim et al. 2013). Previous estimates from instruments such as JCMT suffered from significant blending due to their poor angu- 

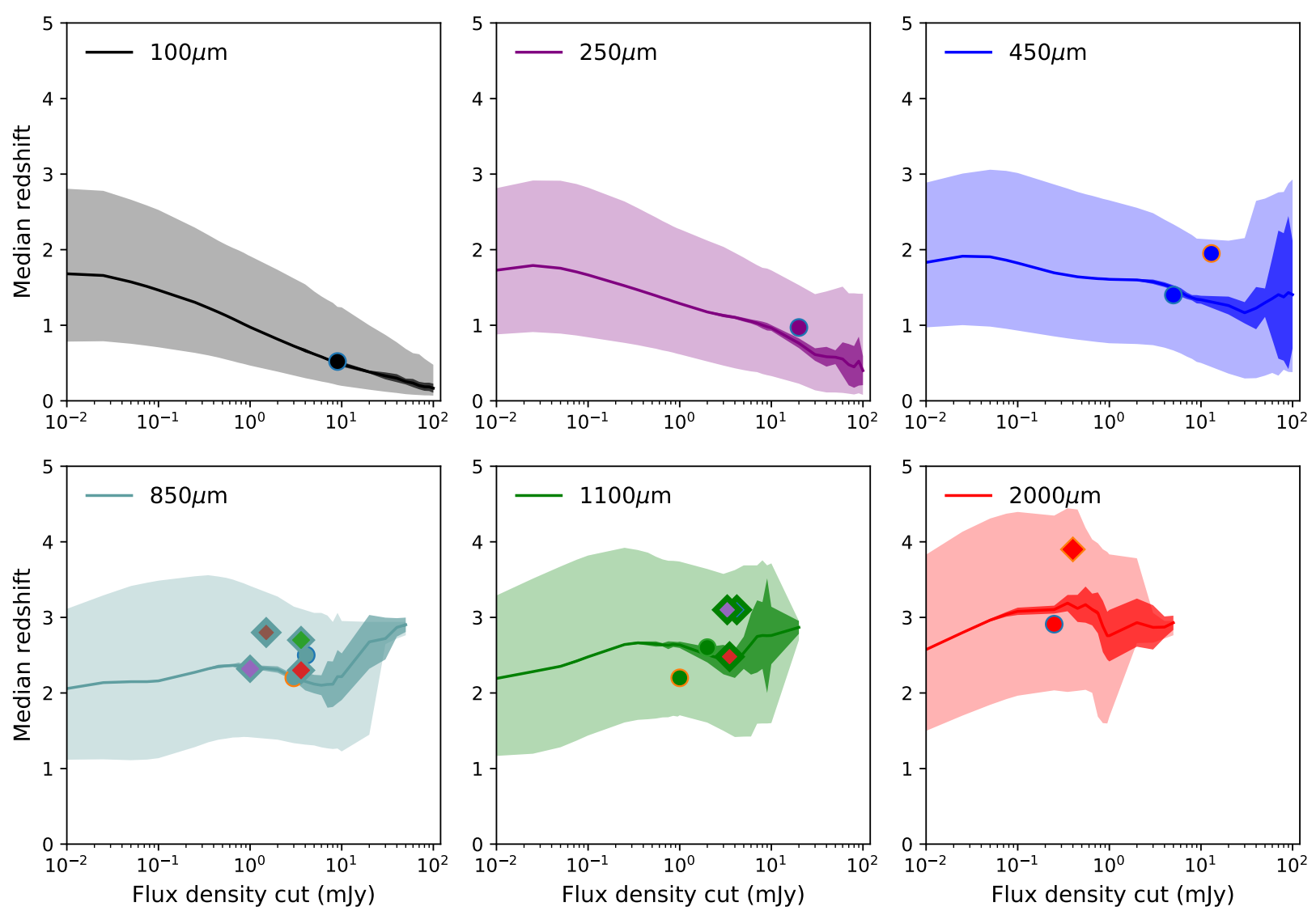

Figure 4. Median redshift as a function of selection flux, for 6 FIR bands from $100 \mu m$ to $2 \mathrm{~mm}$, as labelled in each panel for SHARK. Dark and light shaded regions show the bootstrap error on the median and the $16^{\text {th }}-84^{\text {th }}$ percentile levels, respectively. Symbols in each panel correspond to observational measurements compiled by Hodge \& da Cunha (2020): $100 \mu \mathrm{m}$ and $250 \mu \mathrm{m}$ correspond to Berta et al.

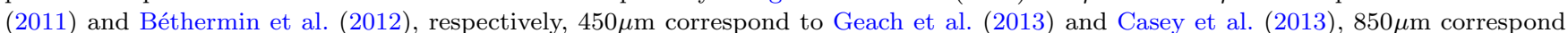
to Wardlow et al. (2011); Chapman et al. (2005); da Cunha et al. (2015); Simpson et al. (2014); Cowie et al. (2018) and Dudzevičiūtė et al. (2020), 1.1mm correspond to Smolčić et al. (2012); Michałowski et al. (2012); Yun et al. (2012); Miettinen et al. (2015); Brisbin et al. (2017) and 2mm corresponds to Staguhn et al. (2014); Magnelli et al. (2019).

lar resolution, forcing simulated lightcones to account for such blending (e.g. Cowley et al. 2015). By comparing with ALMA derived number counts we are able to bypass this issue and compare directly with our predictions.

Fig. 3 shows the number counts of FIR sources in our simulated lightcone from band 9 to band 4 . Observational constraints are currently available for bands 8, 7,6 and 4 (among the bands we can reliably predict SEDs for) and hence the rest serves as predictions. At band 4, we show the observational estimates of Magnelli et al. (2019), which come from the GIZMO instrument rather than ALMA, but are at band 4 wavelength. We find that SHARK predicts numbers counts that are in excellent agreement with observations. There is a significant tension between different observational datasets in band 6 , that is highly apparent at fluxes $<0.7 \mathrm{mJy}$. At brighter fluxes the differences can be attributed to small number statistics and are typically within the errorbars. SHARK predictions lie in between these different observations and within their uncertainties, although its closer to the number counts presented in Fujimoto et al. (2016). The area of our simulated lightcone is more than 4 orders of magnitude larger than what can be accessed with ALMA. For reference, the ALMA large program ASPECS from where the number counts of González-López et al. (2020) come from has an area of $\approx 0.006 \mathrm{deg}^{2}$, while our lightcone has an area of $107 \mathrm{deg}^{2}$. Hence, it is likely that the tension between these different data sets comes from cosmic variance. For reference, using the cosmic variance calculator of Driver \& Robotham $(2010)^{9}$ a survey of the area of ASPECS and in the redshift range of $1<z<3$ has a cosmic variance of $\approx 20 \%$ (however, see Popping et al. 2019 for a counter argument). SHARK predicts a weak upturn in the abundance of galaxies at the bright end. This is caused by bright IR galaxies at low redshift (see Figure 17 of Lagos et al. 2019). Lagos et al. (2019) compared the predicted number counts of SHARK with Herschel observations and there are indications that the abundance of bright galaxies may be too high in the model, perhaps indicating that SHARK tends over-predicts the abundance of local starbursts. This is qualitatively similar to what EAGLE predicts, but in SHARK it manifests to a much lesser degree.

In addition to showing the total number counts in Fig. 3, we also show the contribution from disks and bulges. The

9 Available from http://cosmocalc.icrar.org/. 
latter refer to the components of a galaxy, and hence a single galaxy with both components would contribute to the dotted and dashed lines. In all wavelengths studied in Fig. 3, disks dominate at the faint-end. However, the transition from bulges dominating the number counts to disks happens at fainter fluxes as we move to longer wavelengths. By band6 , we expect the majority of detected galaxies in ALMA to be bulges. Because these bulges are highly star-forming, we refer to them as starbursts below. We break down starbursts into their driving mechanism: galaxy mergers and disk instabilities. Note that from a physical perspective we expect the latter to contain significant rotation (Bournaud et al. 2011) - though this is not modelled in detail in SHARK. At the bright-end of the number counts, both galaxy mergerand disk instability-induced starbursts contribute in similar numbers, while mergers become more prominent at intermediate fluxes. Observations have reported that band- 6 selected SMGs have mixed morphologies with some clearly displaying merger-induced features, while others being more consistent with thick disks (e.g. Hodge et al. 2019; Gullberg et al. 2019). Gullberg et al. (2019) analysed the morphology of 153 bright band- 7 selected SMGs (average band- 7 fluxes of $5 \mathrm{mJy}$ ) and found the sample to have axes ratios and Sèrsic indices that are typical of bars. Seo et al. (2019) in a suite of isolated Milky-Way like hydrodynamical simulations showed that bars can form quickly in gas-rich systems due to dynamical instabilities, and that these bars generally lead to the formation of bulges. If these suggested bars in observations are indeed associated to disk instabilities, then their frequency are consistent with SHARK. The bottom-right panel of Fig. 3 show the redshift distributions of $>0.1$ mJy sources selected in different ALMA bands. There is a systematic shift towards higher redshift when longer wavelengths are used to select galaxies.

The agreement between SHARK and the observations shown in Fig. 3 is unprecedented for galaxy formation simulations, and to our knowledge, SHARK is the first galaxy formation simulation (SAM or hydrodynamical) to reach this level of agreement. We note that our model was not tuned to reproduce galaxy number counts, and our SED construction was simply done using state-of-the-art methods and physical insight from RT calculations of hydrodynamic galaxies. Hence, the agreement of Fig. 3 was not guaranteed and is therefore a true predictive success of our model. We also refer the reader to Lagos et al. (2019) for a comparison with observed number counts and luminosity functions over a wide wavelength range, from the UV to the FIR.

Another independent test of galaxy formation simulations is the redshift distribution of FIR-selected sources. Fig. 4 shows the medians and $16^{\text {th }}-84^{\text {th }}$ percentiles of galaxy samples selected at wavelengths from $100 \mu \mathrm{m}$ to $2 \mathrm{~mm}$ to be brighter than a given flux threshold. By doing this we are assuming that a survey is $100 \%$ complete down to that flux threshold. Symbols show the observational compilation of Hodge \& da Cunha (2020). The only difference with the latter is that we adapted the flux density cut values of Cowie et al. (2018); Magnelli et al. (2019) to better reflect their minimum flux detected. At wavelengths $100 \mu \mathrm{m}$ and $250 \mu \mathrm{m}$, SHARK predicts that the deeper the survey the higher the redshift of the selected galaxies - the brightest sources are therefore more likely to be at lower redshifts. This trend starts to revert at $450 \mu \mathrm{m}$ due to negative k-correction, where
SHARK predicts a very mild dependence of the median redshift of a galaxy survey with its depth. At $850 \mu \mathrm{m}$, SHARK predicts a very flat median redshift dependence on the survey depth, except at the brightest flux density thresholds, where most galaxies are expected to be at higher redshifts (see also bottom-right panel of Fig. 3). At 1.1mm (band-6) and $2 \mathrm{~mm}$ (band 4 ), there is a positive dependence between the median redshift of a survey and its flux selection, so that the highest redshift galaxies are also the brighter ones. The case of $2 \mathrm{~mm}$ (band 4) selected galaxies is interesting, as SHARK shows that a depth of $\approx 0.3 \mathrm{mJy}$ is the optimal one to maximise the chance to have a significant number of galaxies at $z>4$. A much brighter cut would result in the loss of most of the $z>4$ tail. Compared to the observational compilation of Hodge \& da Cunha (2020) we find that SHARK agrees reasonably well - however some systematic differences are worth mentioning. First at band 7 and band 6 , it appears that the mode of the predicted redshift distribution is slightly lower compared to some of the observations. Some observations, however, favour a lower redshift mode, in better agreement with our predictions. At $2 \mathrm{~mm}$ (band 4 ) we also see significant differences in the median redshift estimates of different studies, with SHARK preferring the lower redshift solution. It is also worth highlighting that different methods to measure photometric redshifts yield quite different results. For example, Simpson et al. (2014) and da Cunha et al. (2015) used the same observational sample but obtained median redshifts of 2.3 and 2.5 , respectively. This shows that systematic uncertainties on these redshift distributions are large as they are mostly based on photometric constraints on redshifts rather than spectroscopic confirmation. Redshift campaign follow-ups are thus extremely valuable and necessary to better constrain the simulations.

The broad agreement between the predicted number counts and redshift distributions and observations gives us confidence that we can use SHARK and the lightcone presented here to explore the UV-to-MIR SEDs of FIR-selected galaxies as well as intrinsic properties of SMGs.

\section{UV-TO-MIR PROPERTIES OF SMGS IN SHARK}

An important area of research in SMGs has been the measurement of their panchromatic emission. In order to explore the optical-to-MIR emission of SHARK's SMGs, we analyse the $u, g, r, J, H, K, 4.5 \mu \mathrm{m}, 12 \mu \mathrm{m}$ and $22 \mu \mathrm{m}$ apparent magnitude for band-7 selected sources in Fig. 5. We show as horizontal lines the estimated sensitivity for several existing and future surveys. The magnitude limits of the Hubble Ultra Deep Field (HUDF; Beckwith et al. 2006) are shown in the top three panels; the COSMOS Canada-French Hawaii Telescope (CFHT) survey (Capak et al. 2007) is shown in the top-left panel; the Hyper Suprime-Cam (HSC) Subaru wide and ultra-deep surveys are shown in the top-middle and top-right panels (Aihara et al. 2019); a fiducial James Webb Space Telescope (JWST) survey with the near-infrared camera (NIR) and mid-infrared instrument (MIRI), integrating for 10,000 seconds with each and taking as a threshold a $\mathrm{S} / \mathrm{N}=10$. The latter depth were computed for a point source. Several ALMA large programs have observed in the HUDF and COSMOS regions, and hence these survey limits are of particular interest in this analysis. We find that HUDF 

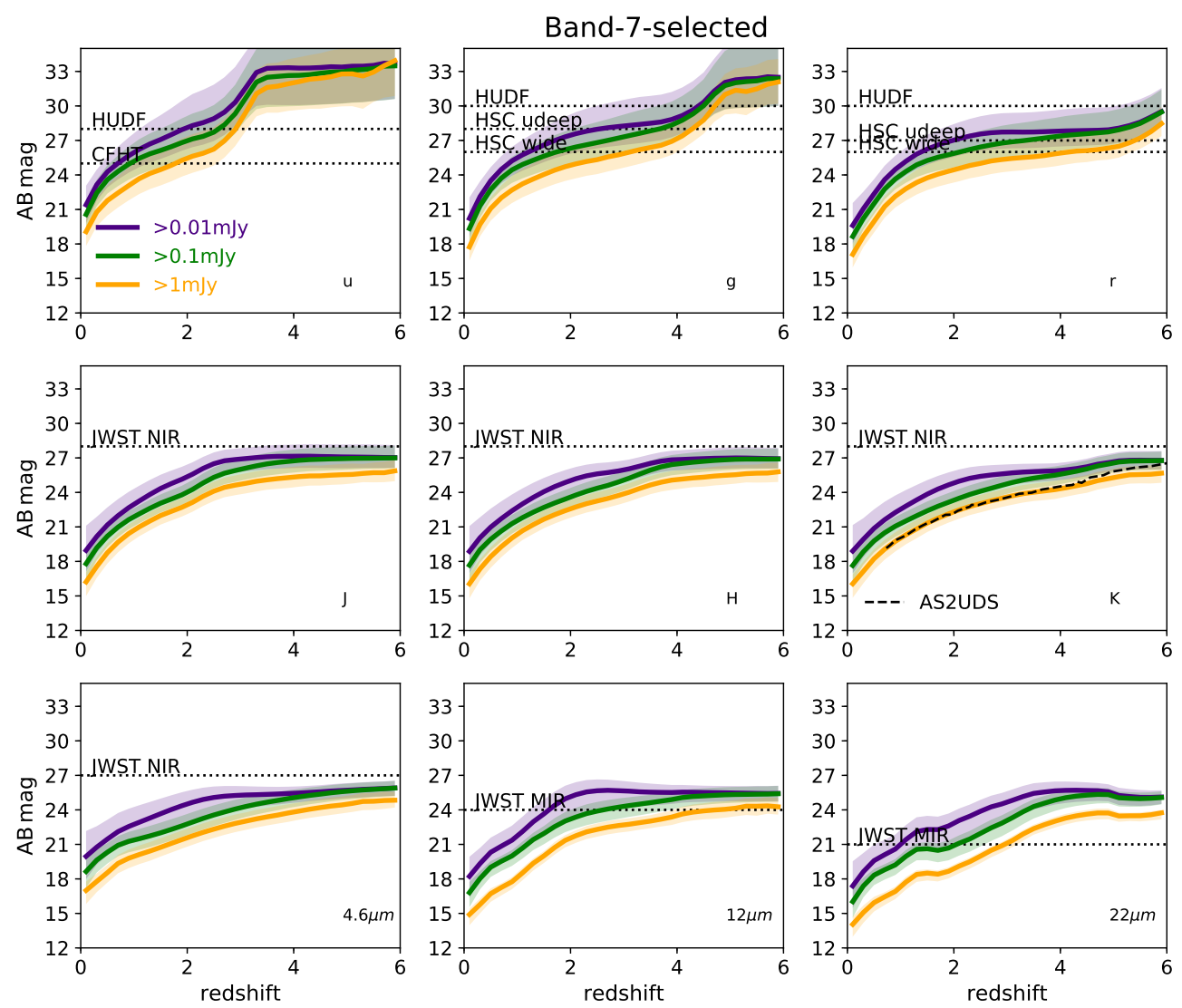

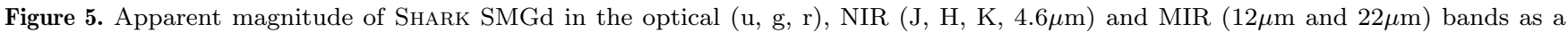
function of redshift. SMGs are selected from their ALMA band-7 continuum emission, adopting 3 different threshold flux above which galaxies are selected (labelled in the top-left panel). Lines with shaded regions show the medians and $16^{\text {th }}-84^{\text {th }}$ percentile ranges, respectively. We show as horizontal lines some various flux limits of relevant surveys, including HUDF (Beckwith et al. 2006), the HSC wide and ultra-deep surveys (Aihara et al. 2019), the COSMOS CFHT survey (Capak et al. 2007), and fiducial 10, 000 seconds integration with the MIRI and NIR JWST instruments. For reference we also show the $K$-band vs. redshift track from the composite SED of the AS2UDS band-7 SMG sample of Dudzevičiūtè et al. (2020), which have a median flux of $\approx 2-4$ mJy, and hence should be comparable to the SHARK sample with $S_{\text {band-7 }}>1$ mJy.

should provide $r$-band counterparts for all SMGs, with the exception of some of the highest redshift, $z>5$, sources with fluxes $>0.01 \mathrm{mJy}$. In the $u$-bands and $g$-bands, however, HUDF would miss all sources with $S_{\text {band }-7}>1$ mJy at $z \gtrsim 3$ and $z \gtrsim 5$, respectively. Going deeper in band-7 flux only makes this worse: sources with $S_{\text {band }-7}>0.01 \mathrm{mJy}$ would have no HUDF $u$ and $g$ counterparts at $z \gtrsim 2$ and $z \gtrsim 4$, respectively.

The HUDF survey comprises a very small area of $0.00127 \mathrm{deg}^{2}$ and hence is not ideal for the study of SMGs (given the small numbers per unit area expected; see Fig. 3). Larger area surveys, such as HSC's wide $\left(1,400 \mathrm{deg}^{2}\right)$, deep $\left(27 \mathrm{deg}^{2}\right)$ and ultra-deep $\left(3.5 \mathrm{deg}^{2}\right)$ surveys likely offer the current best compromise of depth and area for the study of high-redshift sources. The top panels of Fig. 5 show the magnitude limits of HSC's wide and ultra-deep surveys. In the $r$-band, these surveys should provide counterparts for sources with $S_{\text {band-7 }}>1$ mJy at $z \lesssim 4$ and $z \lesssim 6$, respectively; while at the $g$-band, counterparts should be detected for $S_{\text {band-7 }}>1$ mJy sources at $z \lesssim 3$ and $z \lesssim 4.5$, respectively. Focusing on fainter band-7 sources, $S>0.01 \mathrm{mJy}$, we find that HSC's wide (ultra-deep) would provide $g$ and $r$-band counterparts for sources at $z \lesssim 1(z \lesssim 2)$ and $z \lesssim 1.7(z \lesssim 4)$, respectively. In the NIR, a fiducial 10,000 s JWST NIR survey would be capable of comfortably detecting counterparts for all band-7 selected sources, even as deep as $S_{\text {band-7 }}>0.01 \mathrm{mJy}$, in the $J, H$ and $K$ bands, and even up to $z=6$. Similar integration times with JWST's MIRI instrument though would provide counterparts for $S_{\text {band }-7}>1$ mJy sources only at $z \lesssim 4$ at $12 \mu \mathrm{m}$ and $z \lesssim 3$ at $22 \mu \mathrm{m}$. For fainter band-7 sources this becomes worse, typically only detecting counterparts for $S_{\text {band-7 }}>0.01 \mathrm{mJy}$ sources at $z \lesssim 1.5$.

To investigate the consistency of our predicted magnitudes with observations, we turn our attention to the optical-NIR analysis of the ALMA survey AS2UDS sources presented in Dudzevičiūtè et al. (2020). Dudzevičiūtè et al. (2020) analysed sources with band-7 fluxes $>0.6 \mathrm{mJy}$, but with the mode being at $\approx 2-4$ mJy. Below we focus on the IRAC $3.6 \mu \mathrm{m}$ band as Dudzevičiūte et al. (2020) showed this to have the highest completeness. The authors found that $\approx 90 \%$ of their sources had IRAC $3.6 \mu$ m counterparts brighter than $23.5 \mathrm{mag}$. In order to compare with the latter, we select galaxies in our SHARK lightcone to have the 
Table 1. Median stellar mass $\left(M_{\star}\right)$, sSFR, rest-frame V-band attenuation, $A_{\mathrm{V}}$, dust temperature $\left(T_{\text {dust }}\right)$, dust mass $\left(M_{\text {dust }}\right)$ and redshift for SHARK galaxies that in band-7 have a flux $>1 \mathrm{mJy}$ and have IRAC $3.6 \mu \mathrm{m}$ apparent magnitudes $<23.5$ (bright) and $>23.5$ (faint). The errors associated to the medians represent the $16^{\text {th }}$ and $84^{\text {th }}$ percentile ranges.

\begin{tabular}{ccc}
\hline \multicolumn{3}{c}{$S_{\text {band }-7}>1 \mathrm{mJy}$} \\
\hline Property & IRAC $3.6 \mu \mathrm{m}<23.5$ & IRAC $3.6 \mu \mathrm{m}>23.5$ \\
\hline $\log _{10}\left(\mathrm{M}_{\star} / \mathrm{M}_{\odot}\right)$ & $10.44_{-0.39}^{+0.42}$ & $10.05_{-0.34}^{+0.3}$ \\
$\log _{10}\left(\mathrm{sSFR} / \mathrm{Gyr}^{-1}\right)$ & $0.37_{-0.66}^{+0.46}$ & $0.77_{-0.4}^{+0.34}$ \\
$A_{\mathrm{V}} / \mathrm{mag}$ & $1.5_{-0.5}^{+0.55}$ & $2.2_{-0.46}^{+0.55}$ \\
$T_{\text {dust }} / \mathrm{K}$ & $43_{-5}^{+3}$ & $43_{-2.5}^{+1.5}$ \\
$\log _{10}\left(\mathrm{M}_{\text {dust }} / \mathrm{M}_{\odot}\right)$ & $7.9_{-0.75}^{+0.9}$ & $7.5_{-0.57}^{+0.65}$ \\
redshift & $2.2_{-0.8}^{+0.82}$ & $3.5_{-0.65}^{+0.87}$ \\
\hline
\end{tabular}

same band-7 flux distribution and find that $89.9 \pm 1.3 \%$ (error computed from random re-sampling) of galaxies have IRAC $3.6 \mu \mathrm{m}$ emission $<23.5 \mathrm{mag}$, in excellent agreement with the observations. Table 1 shows the median properties of band-7 galaxies with fluxes $>1 \mathrm{mJy}$ that would be IRAC-bright and faint, according to the magnitude threshold above. IRAC-faint SMGs are at significantly higher redshift, have smaller stellar and dust masses (by $\approx 0.5 \mathrm{dex}$ ), higher sSFR (by a factor of 2), and higher rest-frame $V$-band attenuation (by $0.7 \mathrm{mag}$ ) than the IRAC-bright sources. Of all these properties, the ones that seem to be more fundamental in making them IRAC faint are the redshift and the rest-frame $V$-band attenuation. In $\S 5$ we discuss more in general how these properties evolve for galaxies selected in different ALMA bands.

We also show in the middle-right panel of Fig. 5 the expected variation with redshift of the $K$-band magnitude for the composite SED of the AS2UDS sources as presented in Dudzevičiūtè et al. (2020). We find that the latter follows very well our prediction for a band-7 sample with $S_{\text {band-7 }}>$ $1 \mathrm{mJy}$, which is the sample of Fig. 5 that is most closely comparable to the AS2UDS sample. Both these tests show that the SMG galaxy population in SHARK resembles the observations quite closely. Comparisons at other bands (e.g. $U$ or $V$ ) are difficult to reliably carry out due to the high incompleteness of the UDS survey down to the magnitudes investigated in Dudzevičiūtè et al. (2020).

Band- 6 and band- 4 selected sources are shown in Figs. A1 and A2, respectively. In general, at fixed flux and redshift, sources selected in longer-wavelengths have brighter optical, NIR and MIR emission. Hence, for band- 6 or band- 4 selected galaxies, we generally find that existing and upcoming surveys would be able to detect counterparts up to higher redshifts than those found for band-7 selected galaxies.

Because SMGs are highly dust obscured galaxies, their optical colours are expected to be red, potentially contaminating the typical colour-colour selections applied in observations for the selection of passive galaxies at $1 \lesssim z \lesssim 3$ (e.g. Daddi et al. 2004; Brammer et al. 2011; Leja et al. 2019) and even at $z \gtrsim 5$ (Mawatari et al. 2020). This is a related question to that addressed above: what is the optical-to-NIR emission of SMGs in SHARK? We address this by studying the rest-frame $(\mathrm{u}-\mathrm{r})$ vs. $(\mathrm{r}-\mathrm{J})$ colour-colour plane. Bravo et al. (2020) showed that this plane was very efficient at sep-
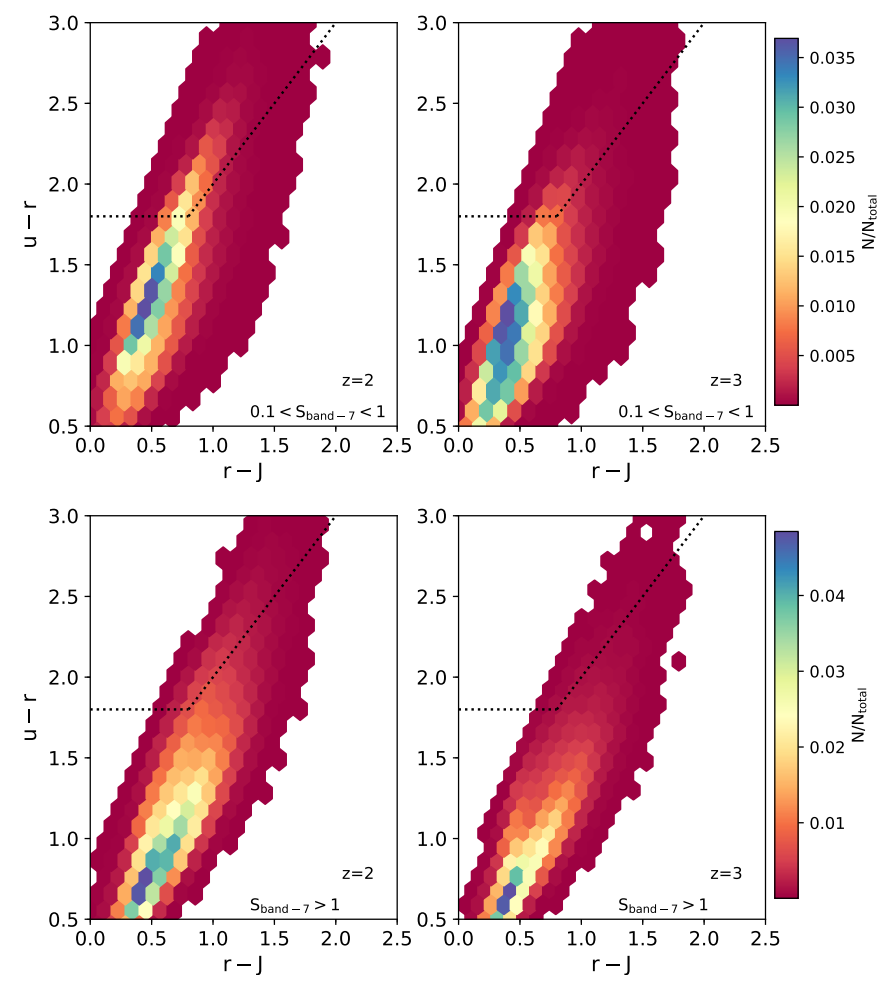

Figure 6. Distribution of band 7 selected galaxies in the rest-frame (u-r) vs. (r-J) plane at redshifts $2 \pm 0.5$ (left) and $3 \pm 0.5$ (right). The top panel shows galaxies selected to have a band-7 flux of $[0.1,1] \mathrm{mJy}$, while the bottom panel showed galaxies with fluxes $>1 \mathrm{mJy}$. The dotted lines show the area that is considered to be populated by passive galaxies based on the analysis of Bravo et al. (2020) of GAMA galaxies at $z<0.5$.

arating passive from star-forming galaxies in the GAMA survey (Driver et al. 2009), and the boundaries used resemble those adopted in high redshift studies (Brammer et al. 2011). Fig. 6 shows the 2D histogram in the above colour-colour plot at $z=2 \pm 0.5$ and $z=3 \pm 0.5$ for galaxies in SHARK selected to have band-7 fluxes in the range $0.1-1 \mathrm{mJy}$ (top panels) and $>1 \mathrm{mJy}$ (bottom panels). We find that $\approx 4 \%$ and $1 \%$ of SHARK galaxies with $0.1 \mathrm{mJy}<\mathrm{S}_{\text {band }-7}<1 \mathrm{mJy}$ would be wrongly classified as passive from their rest-frame $(\mathrm{u}-\mathrm{r})$ vs. $(\mathrm{r}-\mathrm{J})$ colour-colour position at $z=2 \pm 0.5$ and $z=3 \pm 0.5$, respectively. If we instead look at all galaxies that fall in the passive region of the $(u-r)$ vs. $(\mathrm{r}-\mathrm{J})$ colour-colour plane, we find that $42 \%$ and $47 \%$ have $0.1 \mathrm{mJy}<\mathrm{S}_{\text {band }-7}<1 \mathrm{mJy}$ at $z=2 \pm 0.5$ and $z=3 \pm 0.5$, respectively. This percentage is smaller at $z=1,23 \%$, but increases to $50 \%$ at $z=4-5$.

For the sample of SHARK galaxies with $S_{\text {band-7 }}>$ $1 \mathrm{mJy}$, we find that only $1.6 \%$ and $0.4 \%$ of them would be wrongly classified as passive from their $(\mathrm{u}-\mathrm{r})$ vs. $(\mathrm{r}-\mathrm{J})$ colour-colour position at $z=2 \pm 0.5$ and $z=3 \pm 0.5$, respectively. This is much lower than the percentage found for SHARK galaxies with $0.1 \mathrm{mJy}<\mathrm{S}_{\mathrm{band}-7}<1 \mathrm{mJy}$. Of all the galaxies classified as passive, only $1.2 \%$ and $1.5 \%$ have $S_{\text {band }-7}>1 \mathrm{mJy}$ at $z=2 \pm 0.5$ and $z=3 \pm 0.5$, respectively. This percentage decreases mildly towards higher redshift, reaching $0.8 \%$ at $z=5-6$. Appendix $\mathrm{C}$ shows 

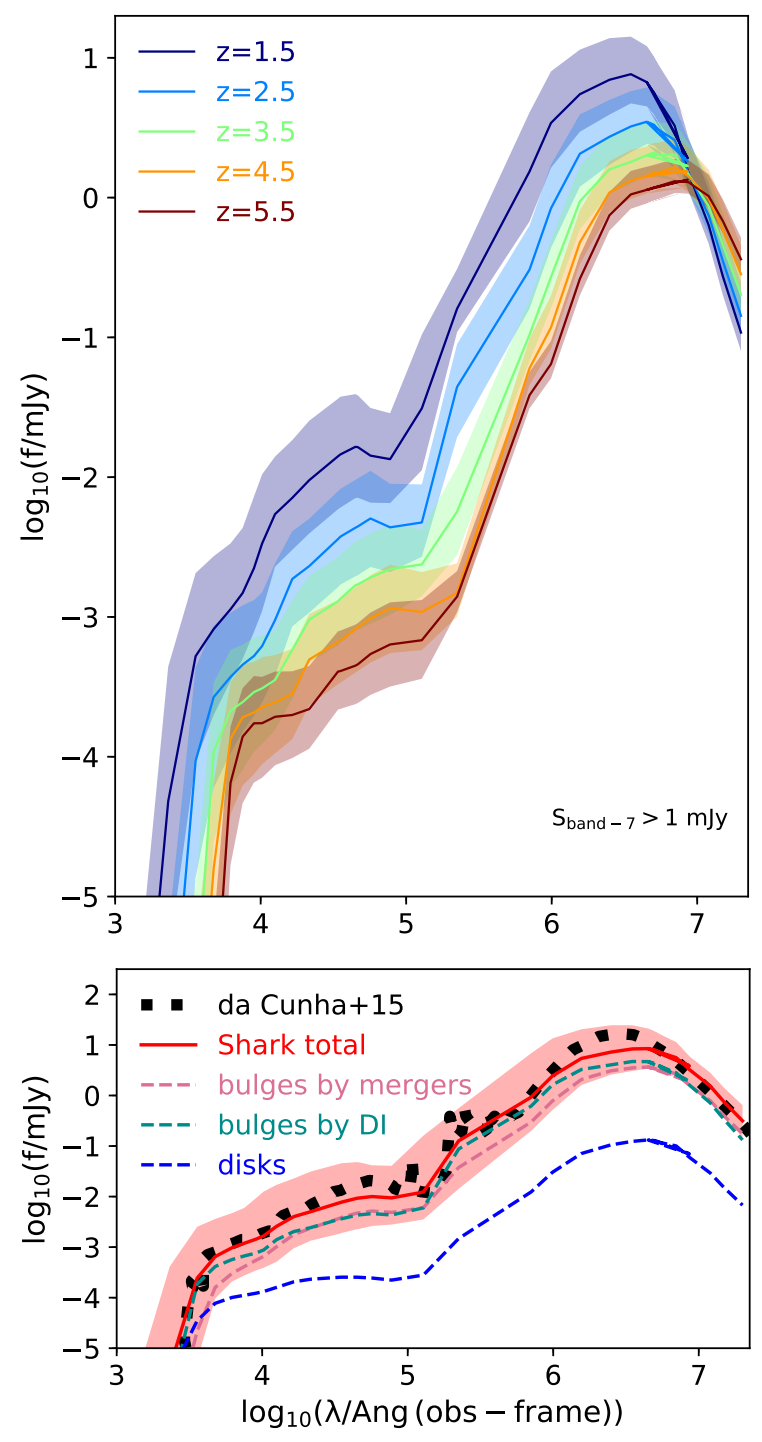

Figure 7. Top panel: Median SEDs of SHARK galaxies selected to have $S_{\text {band }-7}>1 \mathrm{mJy}$ at 5 different redshift bins: $1.5 \pm 0.5$, $2.5 \pm 0.5,3.5 \pm 0.5,4.5 \pm 0.5,5.5 \pm 0.5$, as labelled. Lines and shaded regions show the medians and $16^{\text {th }}-84^{\text {th }}$ percentile ranges. Higher redshift SMGs show on average more absorption in the optical, though significant scatter in seen in the SEDs at the optical-NIR range. Bottom panel: Comparison of the observed ALESS median SED of da Cunha et al. (2015) with an ALESSlike sample of SHARK. The latter is simply selected to have the same flux distribution as the observations. The solid line shows the median, while the shaded region shows the $16^{\text {th }}-84^{\text {th }}$ percentile range of the SHARK prediction. The dashed lines show the median contribution to the total SED from bulges built by galaxy mergers, disk instabilities (both classified as burst mode) and disks, as labelled.

the median intrinsic properties of SHARK $S_{\text {band }-7}>1 \mathrm{mJy}$ galaxies that fall inside/outside the passive region in Fig. 6 . On average the subset that falls in the passive region has higher stellar mass and dust-to-stellar mass ratio, but lower sSFR (though still in the main sequence), compared to those that are outside this region, at fixed redshift.

Put together and considering all galaxies with
$S_{\text {band-7 }}>0.1 \mathrm{mJy}$, the fractions presented above mean that the passive classification in a single colour-colour plane becomes more contaminated as the redshift increases on average, and additional photometric information is therefore required to disentangle truly passive from dust-obscured galaxies (see Leja et al. 2019 for a discussion of alternatives). However, most of the contamination is from mildly dust-obscured galaxies $\left(A_{\mathrm{V}} \approx 0.7-1.2\right)$ rather than highly dust-obscured ones $\left(A_{\mathrm{V}} \approx 1.5-2.1\right)$.

The final investigation we do in this section is the evolution of the average SEDs of galaxies selected to have $S_{\text {band }-7}>1 \mathrm{mJy}$, from $z=1$ to $z=6$. The top panel of Fig. 7 shows the median and the $1 \sigma$ percentile range of the observer-frame FUV-to-FIR SEDs of SHARK galaxies with $S_{\text {band-7 }}>1 \mathrm{mJy}$ in 5 bins of redshift, $z=1.5 \pm 0.5$, $z=2.5 \pm 0.5, z=1.5 \pm 3.5, z=1.5 \pm 4.5, z=1.5 \pm 5.5$. We find that the FUV-to-NIR emission relative to the FIR emission is fainter for higher redshift SMGs, a direct result of the higher average attenuation of the high-redshift SMGs compared to their low redshift counterparts at fixed flux (see middle-left hand panel in Fig. 9; we come back to this in $\S 5)$. We also find that as redshift increases there is less variation in the FIR SED of band-7 SMGs (as seen by the smaller $1 \sigma$ percentile range), but significant variations continue to be seen in the FUV-to-NIR. This then explains why band-7 SMGs span such a wide area in the $(u-r)$ vs. $(r-J)$ colour-colour plane of Fig. 6 .

To compare with the median SED of observed SMGs, we take the band-7 ALESS sample of da Cunha et al. (2015), and select galaxies in SHARK to have the same band-7 flux distribution as the observed galaxies. No other constraint is applied. Hereafter, we refer to this sample as the "ALESSlike SHARK sample". We then compute the median SED of those and the $1 \sigma$ scatter. The bottom panel of Fig. 7 shows our prediction and the observed median SED of galaxies. da Cunha et al. (2015) presented their median SED as a function of rest-frame $\lambda$, which we convert to an observer-frame $\lambda$ by taking the median redshift of the sample, $z=2.2$. The agreement between the observed median SED and our predictions is remarkable. The only difference worth mentioning is the fact that the observed median SED has a slightly brighter FIR peak and at a slightly shorter wavelength than our predicted median, though comfortably within the scatter. We remind the reader though that part of the galaxy SEDs in ALESS does not come from direct measurements, but instead from the best fitting SED. Generally only upper limits are available around the peak of the FIR SEDs of ALESS galaxies.

The bottom panel of Fig. 7 also shows the median contribution from bulges built via disk instabilities and galaxy mergers (both burst mode) and disks. The emission from disks plays a minor role in the median SED of ALESS-like galaxies, except at the FUV. Bulges being built via disk instabilities and galaxy mergers have similar contributions in the NIR and FIR, at wavelengths above the peak, while the former dominates in the NUV-optical and MIR-FIR up to the IR SED peak. This reinforces the idea that the bright band-7 SMGs are an even mix between starbursts driven by galaxy mergers and disk instabilities. At first glance the reader may see a contradiction between the observed morphologies of SMGs (Hodge et al. 2019; Gullberg et al. 2019) and the fact that disks in these ALESS-like galaxies are faint. 
This is not the case and is simply a reflection of the simplicity with which we treat disk instabilities. In SHARK, once a galaxy's disk is found to be globally unstable, we collapse it instantaneously and drive a starburst with the available gas that typically takes a few $100 \mathrm{Myr}$ to exhaust. In detailed hydrodynamical simulations (Bournaud et al. 2010; Seo et al. 2019) however, this process of gas inflows and bulge formation is not instantaneous, and may take several hundred Myr (similar timescale to the molecular gas depletion) - hence the contribution from disk instability-driven bulges should be seen as the combined contribution of the disks+bulges of those galaxies.

Overall we find that SHARK is capable of reproducing remarkably well current constraints on the optical-to-FIR emission of SMGs. This adds weight to our predictions for what upcoming surveys, for example those performed with JWST, will be able to offer.

\section{INTRINSIC PROPERTIES OF SMGS IN SHARK ACROSS COSMIC TIME}

In this section we focus on intrinsic properties of galaxies selected in ALMA bands 7, 6 and 4, as a function of redshift, including their environment (as measured by their host halo mass).

Fig. 8 shows the median stellar mass, sSFR and halo mass as a function of redshift for galaxies selected in ALMA bands 7, 6 and 4 and using three flux thresholds of $>$ $0.01 \mathrm{mJy},>0.1 \mathrm{mJy}$ and $>1 \mathrm{mJy}$. We also show the $1 \sigma$ scatter around the medians as shaded regions. For the band7 selected galaxies we also plot the stellar mass and sSFR of the AS2UDS sample of Dudzevičiūtè et al. (2020) and the ALESS sample of da Cunha et al. (2015), and the SMGs host halo mass inferred from clustering by Hickox et al. (2012). For band 7, we show the medians for our SHARK ALESSlike sample (see bottom panel of Fig. 7). We also show for reference the sSFR evolution of MS galaxies in SHARK. The latter is computed as the median SSFR of central galaxies with stellar masses $10^{9}-10^{10} \mathrm{M}_{\odot}$, which are vastly dominated by star-forming galaxies in SHARK. Fig. 9 shows the median dust mass, effective dust temperature and rest-frame attenuation $A_{\mathrm{V}}$ as a function of redshift for the same SHARK galaxies of Fig. 8. We also show the inferred properties of ALESS galaxies presented in da Cunha et al. (2015) using MAGPHYS (da Cunha et al. 2008) in the left-hand panels. Fig. 10 shows the median fractional contribution of starbursts to the total SFR of galaxies, the SFR-weighted effective radii and the $\mathrm{CO}(1-0)$ brightness luminosity as a function of redshift for the same galaxies of Fig. 8. Below we analyse the relevant trends and predictions of these figures property by property.

\subsection{Stellar masses of SMGs}

Focusing first on stellar mass (top panels of Fig. 8), we find the stellar mass to increase with increasing band-7, 6 and 4 flux. At fixed flux, band- 4 galaxies are more massive than band-7 galaxies. In fact, band-4 galaxies with $S_{\text {band-4 }}>$ $1 \mathrm{mJy}$ trace the most massive galaxies in the simulation at all redshits. This is seen when comparing the median for galaxies with $S_{\mathrm{band}-4}>1 \mathrm{mJy}$ with the median of the
100 most massive galaxies in the lightcone as a function of redshift (dashed line in the top panels). Note that the latter peaks at $z \approx 1$ (rather than $z=0$ ) due to the limited volume probed by the lightcone at lower redshifts compared to the total volume of the simulation. This is in part responsible for the peak stellar mass we see in all the presented selections in bands 7 and 6 ; albeit the latter happens at slightly lower redshift, $z \approx 0.7$. The decrease in median stellar mass at $z>$ 0.7 in all cases is slower than the decrease in the stellar mass of the 100 most massive galaxies, which is a consequence of ALMA bands 7, 6, 4-selected galaxies tracing more massive galaxies relative to the break of the stellar mass function as the redshift increases. At $z>4$ all massive galaxies are $m m$ bright, which is clear from the $S_{\text {band-4 }}>1$ mJy sample overlapping with the dashed line in the top-right panel.

Compared to observations, both AS2UDS (Dudzevičiūte et al. 2020) and ALESS (da Cunha et al. 2015) are consistent with the upper $84^{\text {th }}$ percentile of the sample with $S_{\text {band-7 }}>1 \mathrm{mJy}$. This is not necessarily surprising, as both samples have median band- 7 fluxes of $\approx 2-4 \mathrm{mJy}$. The median of the SHARK ALESS-like sample is indeed higher than the $S_{\text {band- } 7}>1$ mJy sample by $\approx 0.2$ dex in better agreement with the observational estimates. A large source of uncertainty in the measured properties of SMGs shown here is the lack of a secured redshift, which is a large systematic effect in stellar masses. If the redshifts were slightly overestimated, the stellar masses of the observed SMGs would be lower and closer to the SHARK predictions.

\subsection{The Specific Star Formation Rates of SMGs}

Focusing on sSFRs (middle panels of Fig. 8), we find that the brighter the galaxy in ALMA bands 7, 6, 4, the higher the sSFR, with galaxies selected at longer wavelengths having higher sSFRs at fixed flux. Quantifying this in terms of the distance to the MS, we find that bands 7, 6, and 4 selected galaxies with $S>1 \mathrm{mJy}$ have median $\mathrm{sSFR} / \mathrm{sSFR}_{\mathrm{MS}} \approx 2$, $\mathrm{sSFR} / \mathrm{sSFR}_{\mathrm{MS}} \approx 3$ and $\mathrm{sSFR} / \mathrm{sSFR}_{\mathrm{MS}} \approx 7$, respectively. This means that galaxies with $S_{\text {band }-7}>1$ mJy and $S_{\text {band-6 }}>1$ mJy are only mildly above the MS, and applying some typical selection criteria in the literature they may even be considered MS - for example Béthermin et al. (2015) adopt $0.25<\mathrm{sSFR} / \mathrm{sSFR}_{\mathrm{MS}}<4$ to define galaxies as MS. Only band- 4 galaxies with $S_{\text {band }-4}>1 \mathrm{mJy}$ would truly be considered starbursting objects by the latter definition. Note that SHARK galaxies with $S_{\text {band }-7}>0.1 \mathrm{mJy}$ and $S_{\text {band-6 }}>0.1 \mathrm{mJy}$ are MS galaxies at $z \lesssim 2$ and only mildly above the MS at higher redshifts, $\mathrm{sSFR} / \mathrm{sSFR}_{\mathrm{MS}} \approx 1.3-2$. In band- 4 , galaxies with $S_{\text {band-4 }}>0.1 \mathrm{mJy}$ are more extreme, with $\mathrm{sSFR} / \mathrm{sSFR}_{\mathrm{MS}} \approx 2-3.5$. Note that one would have to go down to fluxes of $S \approx 0.01$ mJy in ALMA bands 7 , 6 and 4 to trace typical MS galaxies across the whole redshift range studied here. Compared to AS2UDS and ALESS, we find that the predicted sSFR of ALESS-like SHARK galaxies agrees well with the observations (within the scatter). There is a hint of a trend of sSFR decreasing with increasing redshift in the observations, but is highly uncertain given the large errorbars. SHARK predicts the opposite: the sSFR of ALMA band selected galaxies increases with increasing redshift at fixed flux, in a way that mimics the evolution of the MS normalisation. 

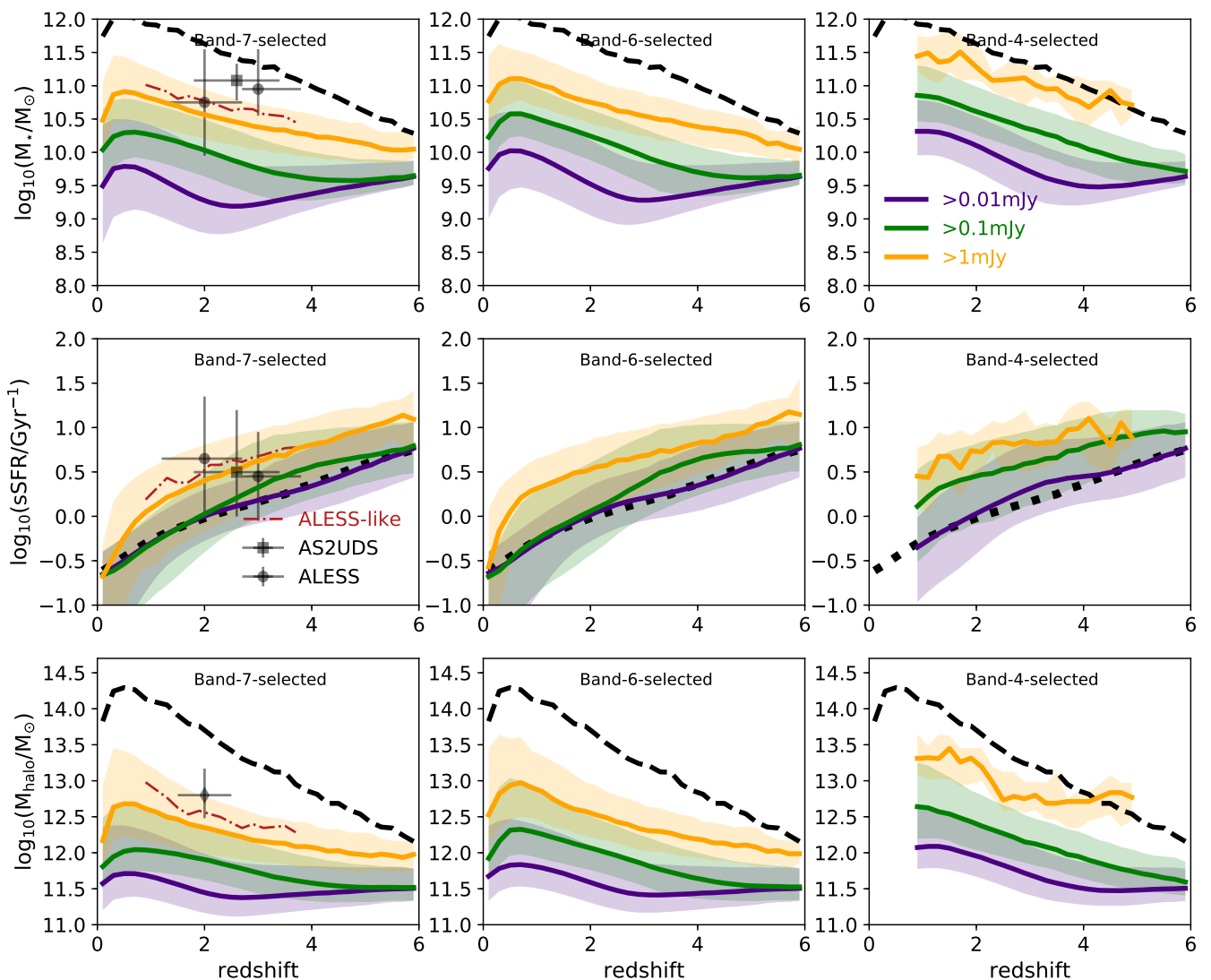

Figure 8. Stellar masses, sSFRs and halo masses for the SMGs of Figs. 5, A1 and A2 as a function of redshift. Lines with shaded regions show the medians and $16^{\text {th }}-84^{\text {th }}$ percentile ranges, respectively. For reference, the dashed lines in the top and bottom panels show the median mass of the 100 most massive objects (in stellar and halo mass, respectively) in the simulated lightcone; the dotted lines in the middle panels show the sSFR evolution of galaxies with $10^{9} \mathrm{M}_{\odot}<\mathrm{M}_{\star}<10^{10} \mathrm{M}_{\odot}$ in SHARK that have SFR $>0$; and the dot-dashed lines in the left hand panels show the median for the SHARK ALESS-like sample of the bottom panel of Fig. 7. We also show the average properties of the AS2UDS (Dudzevičiūtè et al. 2020) and of the ALESS (da Cunha et al. 2015) band-7 SMGs in the top and middle-left panels, as labelled. For ALESS, we show the two redshift bins presented in da Cunha et al. (2015). At the bottom panel we show the hot halo mass inference of Hickox et al. (2012) (diamond) from the spatial clustering of SMGs. All these datasets are biased towards bright band-7 SMGs, $\gtrsim 2 \mathrm{mJy}$, and hence are more closely comparable to the SHARK sources with $S_{\text {band-7 }}>1 \mathrm{mJy}$.

\subsection{The host halo masses of SMGs}

The environment of SMGs has been a topic of great interest in the literature. Hickox et al. (2012) measured the spatial clustering of SMGs selected from the $870 \mu \mathrm{m}$ Large APEX Bolometer Camera (LABOCA) instrument, that have fluxes $\gtrsim 4 \mathrm{mJy}$, and from the clustering biases inferred the host halo masses to be $\approx 10^{12.8} \mathrm{M}_{\odot}$. Blending of sources due to the poor angular resolution of LABOCA may decrease this host halo mass by a factor of $\approx 2-6$ to $\approx 10^{12}-10^{12.5} \mathrm{M}_{\odot}$ (Cowley et al. 2017b).

The bottom panels of Fig. 8 show the median host halo mass for galaxies selected in bands 7,6 and 4 and using three different threshold fluxes. We also show for reference the median halo mass of the 100 most massive halos as a function of redshift. We generally find that brighter galaxies in the three ALMA bands analysed here inhabit more massive halos. The difference in the median mass for the faintest and brightest flux thresholds increases from band-7 to band-4: galaxies with $S>0.01 \mathrm{mJy}$ reside in halo masses $\approx 0.8 \mathrm{dex}, \approx 1.2$ and $\approx 1.5$ dex less massive than galaxies with $S>1$ mJy in bands 7,6 and 4 , respectively. We also find that band- 4 selected galaxies reside in more massive halos at fixed flux than band 6 and 7 galaxies. In fact, at $z>3.5, S_{\text {band-4 }}>1 \mathrm{mJy}$ galaxies are excellent tracers of the most massive halos, and hence represent a unique probe of proto-clusters, given the expected correlation between the mass of progenitor halos and their $z=0$ descendants (Muldrew et al. 2015). Casey et al. (2019) in a blind ALMA band 4 survey detected a bright source, $S_{\text {band }-4} \approx 0.7 \mathrm{mJy}$, at $z \approx 5.8$ that is consistent with being a proto-cluster of mass $\approx 10^{12.5} \mathrm{M}_{\odot}$, providing a tentative confirmation of our prediction.

The median host halo mass of galaxies with $S>1 \mathrm{mJy}$ vary little with redshift $\lesssim 0.5 \mathrm{dex}$, while the overall evolution of the halo mass function is evolving much more strongly. For reference, at a fixed number density of $10^{-3} \mathrm{~h}^{3} \mathrm{cMpc}^{-3}$, the halo mass evolves by $\approx 1.5$ dex from $\approx 10^{12.4} \mathrm{M}_{\odot} \mathrm{h}^{-1}$ at $z=0$ to $\approx 10^{10.9} \mathrm{M}_{\odot} \mathrm{h}^{-1}$ at $z=6$ (in a $\Lambda \mathrm{CDM}$ universe). This means that the clustering bias of these ALMAselected SMGs is increasing with increasing redshift. This is also clear from the way the median halo mass of band-7 and 6 selected galaxies with $S>1 \mathrm{mJy}$ approaches the mass of the 100 most massive halos in the lightcone at $z \rightarrow 6$. We 

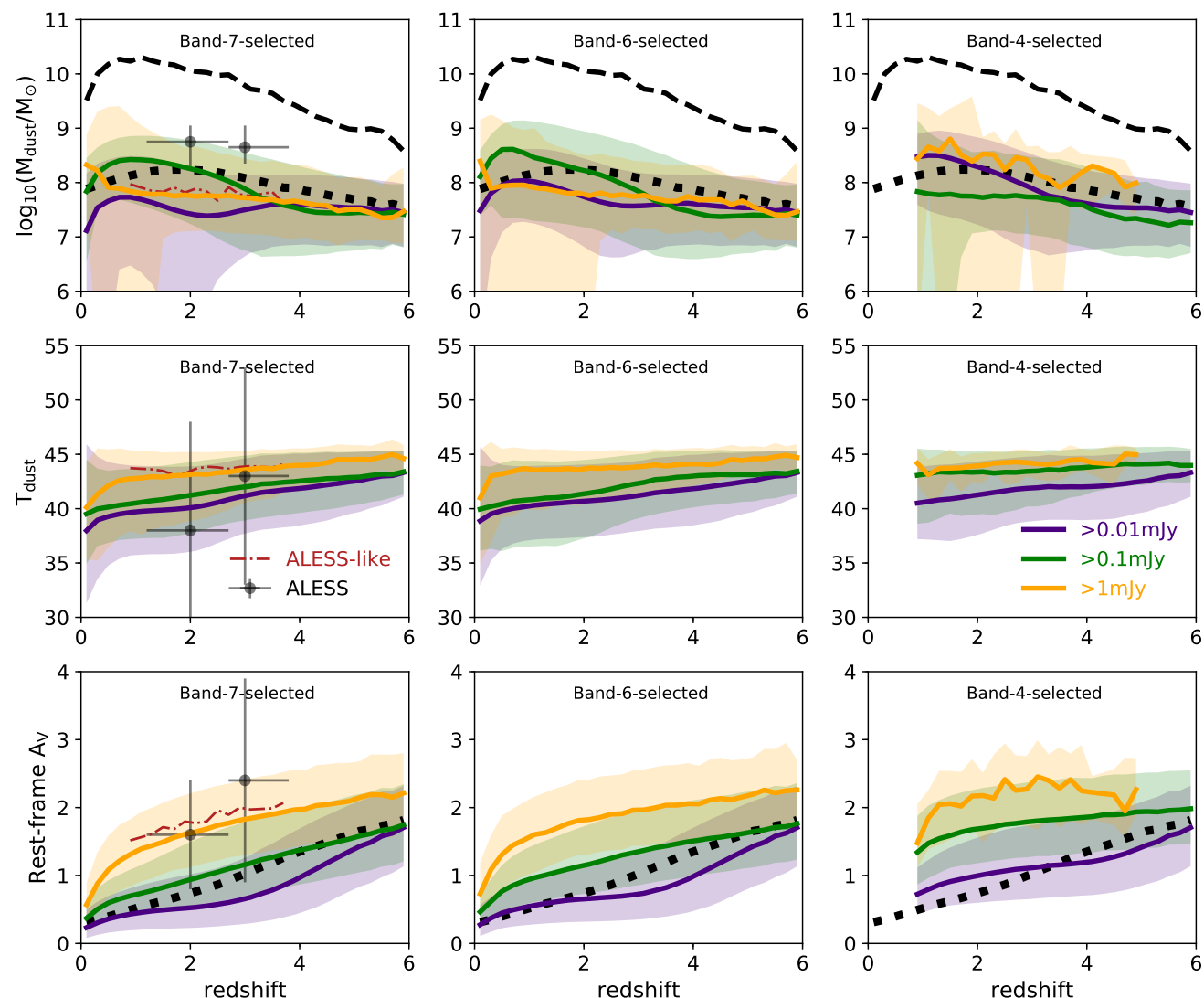

Figure 9. As in Fig. 8 but for dust mass, dust temperature and rest-frame attenuation in the V-band. We include the observational constraints of da Cunha et al. (2015), which are meant to be comparable to the band- 7 sources that are brighter than 1 mJy. The dotted lines show the median dust mass (top panels) and $A_{\mathrm{V}}$ (bottom panels) of the MS galaxies shown in middle panels of Fig. 8; dot-dashed lines in the left hand panels show the median for the SHARK ALESS-like sample of the bottom panel of Fig. 7; and dashed lines in the top panels show the median dust mass of the 100 most dust massive galaxies.

show the observational estimate of Hickox et al. (2012) in the bottom, left panel of Fig. 8 and found it to be consistent with the predicted median halo mass of ALESSlike SHARK galaxies, within the errorbars. The small difference in the latter comparison can simply be due to the sample of Hickox et al. (2012) being brighter than ALESS and/or source confusion (Cowley et al. 2017b). Fujimoto et al. (2016) computed an upper limit for the typical halo mass hosting $S_{\text {band-6 }} \gtrsim 0.1$ mJy sources using the countsin-cell method of $10^{12.5} \mathrm{M}_{\odot}$ consistent with our predicted median halo mass for those galaxies, $10^{12} \mathrm{M}_{\odot}$.

\subsection{Dust masses, temperatures and rest-frame optical attenuation of SMGs}

The top and middle panels of Fig. 9 present the dust mass and temperature evolution with redshift for galaxies selected in ALMA bands 7, 6, 4 to have fluxes $>0.01 \mathrm{mJy},>0.1 \mathrm{mJy}$ and $>1 \mathrm{mJy}$. In bands 6 and 7 , the dust mass correlates nonlinearly with FIR flux, with the $S>0.1$ mJy sample having the highest dust masses on average, of the three flux threshold samples. The $S>1 \mathrm{mJy}$ in these bands have on average $\lesssim 0.3$ dex less dust mass than the $S>0.1 \mathrm{mJy}$ galaxies, and similar to the faint sample $S>0.01 \mathrm{mJy}$. This is a direct consequence of the fact that in SHARK MS galaxies have the highest dust masses at fixed stellar mass (see bottom panels of Fig. 1), with the dust mass decreasing as one moves above the MS. However, in band 4 the $S_{\text {band-4 }}>1$ mJy galaxies also have higher dust masses. This is due to the fact that those galaxies correspond to the most massive, starbursting galaxies in SHARK, which also have large amounts of dust (see galaxies above the MS and with $M_{\star}>10^{11} \mathrm{M}_{\odot}$ in the bottom panels of Fig. 1). In any case, neither of these ALMA bands selected samples trace the most dust massive galaxies (see dashed lines).

In the middle panels of Fig. 9 we show the evolution of $T_{\text {dust }}$. There is a weak evolution of an increasing $T_{\text {dust }}$ with increasing redshift for the faint and intermediate flux samples $(S>0.01 \mathrm{mJy}$ and $>0.1 \mathrm{mJy}$, respectively), while the brightest samples $(S>1 \mathrm{mJy})$ display almost no evolution, with $T_{\text {dust }}$ being $\approx 42-44 \mathrm{~K}$ at all redshifts. Fig. 1 shows that $T_{\text {dust }}$ varies with both $M_{\star}$ and SFR, but at high $M_{\star}$ and SFRs typical of the galaxies traced in the selections of Fig. $9, T_{\text {dust }}$ is always $\approx 40-45 \mathrm{~K}$. This behaviour in SHARK is due to the underlying assumption that dust in its diffuse and birth cloud phases have two distinct but constant temperatures. Note that the ALESS-like SHARK sample has dust masses and temperatures consistent with the $S_{\text {band-7 }}>1$ mJy sample, despite its average brighter flux. The high $T_{\text {dust }}$ that we find for SMGs indicate that dimming 
due to the cosmic microwave background (da Cunha et al. 2013; Jin et al. 2019) is not expected to have an important effect in our predictions and hence can be neglected.

Compared to the derived physical properties of ALESS galaxies presented in da Cunha et al. (2015), we find that SHARK predicts dust masses that are too low by $0.5-1$ dex, which is compensated by the slightly higher dust temperatures. In a grey body, the bolometric IR luminosity, $L_{\mathrm{IR}} \propto$ $M_{\text {dust }} T_{\text {dust }}^{4+\beta}$, with typical values of $\beta \approx 1-3$. Because the dependence with temperature is much stronger, at fixed luminosity, large variations in dust mass can be compensated by small variations in temperature. Interestingly, the bottom panel of Fig. 7 shows that the median SHARK SED of ALESS-like galaxies is very similar to the observed SED reported in da Cunha et al. (2015). This suggests that the differences seen in the top and middle panels of Fig. 9 are at least in part due to the systematic effects introduced in fitting the SEDs of galaxies to infer dust masses and temperatures. Inferences of dust temperature in dusty galaxies have not reached consensus, with some other studies indicating $T_{\text {dust }} \approx 50 \mathrm{~K}$ to be preferred (Jin et al. 2019; Cortzen et al. 2020; Yang et al. 2020). Adopting the latter temperature (instead of the $\approx 40 \mathrm{~K}$ found in da Cunha et al. 2015) and $\beta=1$ and $=3$, one would expect a reduction in the dust mass of a factor 3 and 4.7, respectively, in better agreement with our predictions.

The bottom panels of Fig. 9 show the evolution of the rest-frame $A_{\mathrm{V}}$ for SHARK galaxies selected in ALMA bands 7, 6 and 4. The FIR flux of galaxies is correlated with $A_{\mathrm{V}}$ so that a higher ALMA band flux is associated to a higher $A_{\mathrm{V}}$ at fixed redshift. In any one of the flux selections, $A_{\mathrm{V}}$ increases with increasing redshift. We find that the samples with $S>1$ mJy have $A_{\mathrm{V}}>1$ at $z>0.3$ in all the analysed ALMA bands, and on average $A_{\mathrm{V}}$ is higher for galaxies selected at longer wavelengths and at fixed flux (i.e. $S_{\text {band-4 }}>1$ mJy galaxies have a higher $A_{\mathrm{V}}$ than $S_{\text {band-7 }}>1 \mathrm{mJy}$ at fixed redshift). For reference, the dotted line shows the $A_{\mathrm{V}}$ evolution of MS galaxies with $10^{9} \mathrm{M}_{\odot}<\mathrm{M}_{\star}<10^{10} \mathrm{M}_{\odot}$. SHARK galaxies with $S_{\text {band-7 }}>0.1$ mJy follow closely the $A_{\mathrm{V}}$ evolution of MS galaxies of masses $10^{9} \mathrm{M}_{\odot}<\mathrm{M}_{\star}<10^{10} \mathrm{M}_{\odot}$. In comparison, band- 6 and 4 galaxies selected using the same flux threshold are above the MS $A_{\mathrm{V}}$ values. Compared to the observations, we find that the ALESS-like SHARK galaxies produce consistent $A_{\mathrm{V}}$ values within the uncertainties, and show a qualitatively similar evolutionary trend. This is one of the main reason why the FUV-to-NIR part of the median SED of ALESS-like SMGs agree well with observations (bottom panel of Fig. 7).

Together with $A_{\mathrm{V}}$ evolving with redshift, the slope of the attenuation curve $\eta$ in Eqs. 1 and 2 is also changing. We measure an effective $\eta_{\text {eff }}$ following Eq. 3, but instead of temperature we use $\eta_{\mathrm{ISM}}$ and $\eta_{\mathrm{BC}}$. We find that $\eta_{\mathrm{eff}}$ increases from -0.77 at $z=0$ to -0.45 at $z=6$ for the galaxy sample with $S_{\text {band-7 }}>1 \mathrm{mJy}$. This means that attenuation curves become greyer with increasing redshift, in agreement with the RT analysis of hydrodynamical simulations by Narayanan et al. (2018).

\subsection{Star formation modes in SMGs}

The top panels of Fig. 10 show the fractional contribution of the burst mode of SF to the total instantaneous SFR of galaxies as a function of redshift for three flux samples selected in bands 7, 6 and 4. We remind the reader that the burst mode of SF can be triggered by both galaxy mergers and global disk instabilities. In both cases gas is driven towards the centre of the galaxy and a starburst takes place from that gas reservoir. The SF law applied in the burst mode is the same as the normal mode of SF, except for a 10 times shorter molecular gas depletion time (see $\S 2.2$ for a description).

Galaxies with $S>1$ mJy in bands 7,6 and 4 have SFRs dominated by the burst mode at $z \gtrsim 0.7, z \gtrsim 0.4$ and throughout the whole redshift range, respectively. However, from Fig. 3 and the bottom panel of Fig. 7, we know that this burst mode is split between galaxy merger- and disk instabilities-driven in a ratio close to 50-50. In the case of galaxies with $S>0.1 \mathrm{mJy}$, we find that their SFR is dominated by the burst mode at $z \gtrsim 2.5, z \gtrsim 1.5$ and across the whole redshift range for galaxies selected in band 7,6 and 4 , respectively. In the faintest sample $S>0.01 \mathrm{mJy}$, we find their SFR to be dominated by the burst mode at $z \gtrsim 4$ for bands 7 and 6 and $z \gtrsim 2.5$ for band 4 . For reference, MS galaxies with stellar masses $10^{9}-10^{10} \mathrm{M}_{\odot}$ have their SFR dominated by bursts at $z \gtrsim 3$. This means that only pushing down to fluxes of $0.01-0.1 \mathrm{mJy}$ one is able to get a sample that is representative of MS galaxies whose sub-mm emission is induced by both starbursts and 'normal' SF (see also the middle panel of Fig. 8). This is extremely challenging even with ALMA.

\subsection{Galaxy sizes of SMGs}

Observations with ALMA of SMGs have revealed rather compact galaxies (with FIR continuum sizes $1-5 \mathrm{kpc}$; e.g. see Ikarashi et al. 2015; Hodge et al. 2016; Oteo et al. 2017; Fujimoto et al. 2017). However, SMGs do not appear necessarily more compact than high-z MS galaxies (Elbaz et al. 2018; Gómez-Guijarro et al. 2019; Puglisi et al. 2019), and it appears like these sizes do not depend on merger state (Fujimoto et al. 2017). Fujimoto et al. (2017) found a weak trend of galaxy sizes to increase with increasing FIR luminosity, with galaxies of $L_{\mathrm{FIR}} \approx 10^{12} \mathrm{~L}_{\odot}$ and $L_{\text {FIR }} \approx 10^{13} \mathrm{~L}_{\odot}$ having effective radii $\approx 1 \mathrm{kpc}$ and $\approx 2 \mathrm{kpc}$, respectively. Here, we explore the SFR-weighted effective radii, $r_{\text {gal }}$, of galaxies in SHARK selected using three different flux thresholds in ALMA bands 7, 6 and 4. These are presented in the middle panels of Fig. 10. We compute $r_{\text {gal }}=\left(\mathrm{SFR}_{\text {disk }} \times \mathrm{r}_{50, \text { disk }}+\mathrm{SFR}_{\text {bulge }} \times \mathrm{r}_{50 \text {, bulge }}\right) / \mathrm{SFR}_{\text {total }}$, where $\mathrm{SFR}_{\text {disk }}$ and $\mathrm{SFR}$ bulge are the SFRs contributed by the disk and bulge, respectively, to $\mathrm{SFR}_{\text {total }}$, and $r_{50 \text {,disk }}$ and $r_{50, \text { bulge }}$ are the half-gas mass radii of the disk and bulge, respectively.

Overall, we find that $r_{\text {gal }}$ decreases with increasing redshift at fixed flux and a weak dependence of $r_{\text {gal }}$ on the observed flux at fixed redshift, so that brighter galaxies in the submm have on average larger $r_{\text {gal }}$, in qualitative agreement with Fujimoto et al. (2017). However, the latter is very weak and almost completely disappears for galaxies at $z \gtrsim 3$. The only galaxy sample that is clearly more extended throughout 

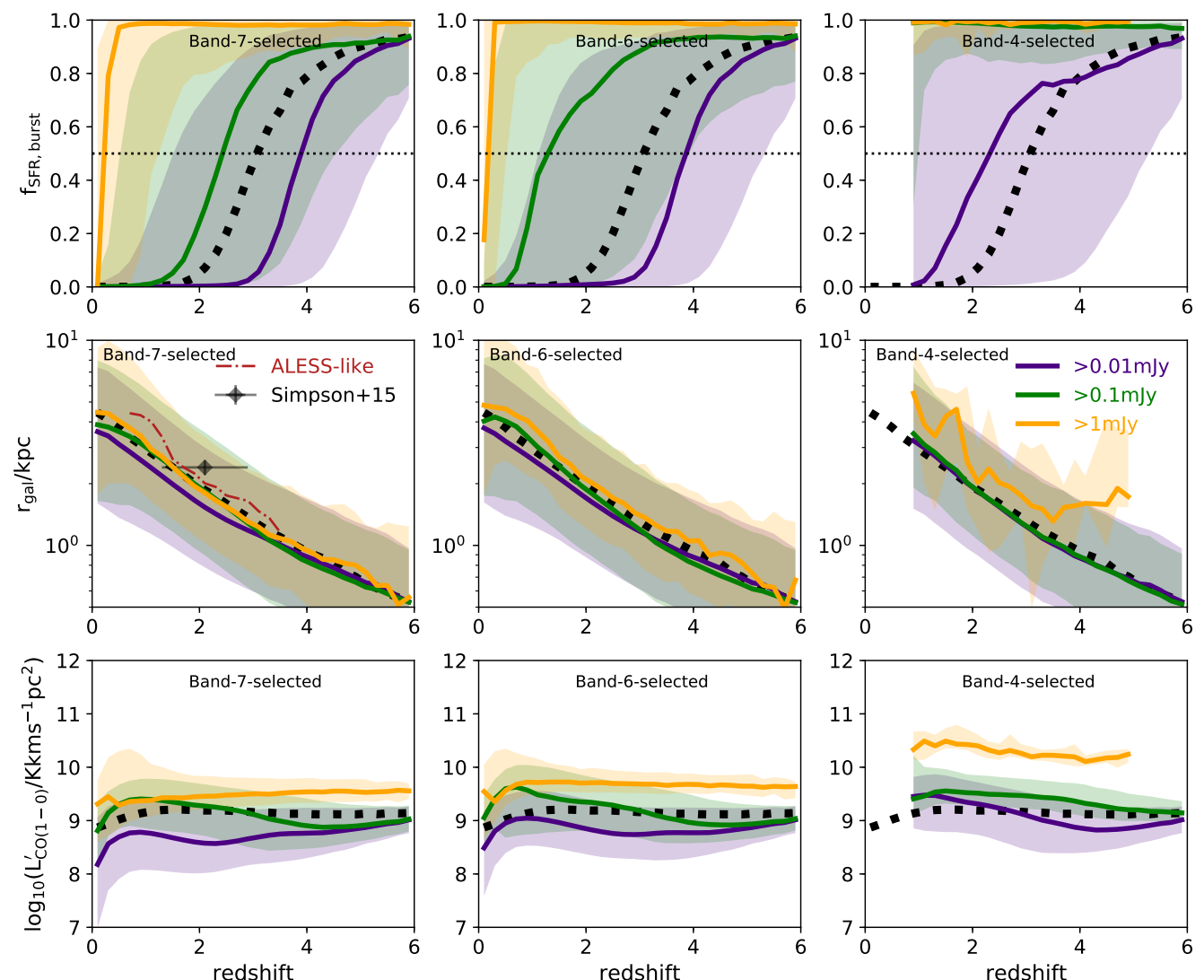

Figure 10. As in Fig. 8 but for the fraction of SFR that is in the form of a starburst, the physical SFR-weighted effective radius of galaxies, and the $\mathrm{CO}(1-0)$ brightness luminosity. In each panel, the dotted lines show the evolution of the median of MS galaxies defined as in Fig. 8. In the middle, left hand panel, we also show the observational estimates of Simpson et al. (2015) for the ALESS sample, together with the median of the SHARK ALESS-like sample of the bottom panel of Fig. 7.

the whole redshift range is that with $S_{\text {band-4 }}>1 \mathrm{mJy}$. For reference, we also show the size evolution of MS galaxies with stellar masses $10^{9}-10^{10} \mathrm{M}_{\odot}$ and find that those are indistinguishable from band 7 and 6 bright galaxies, $S>1 \mathrm{mJy}$, in agreement with the conclusions in Barro et al. (2016); Elbaz et al. (2018).

The sizes of galaxies with $S>1$ mJy in bands 7 and 6 (which are the bands used for the observational studies above) are within the observed ones, with typical values of $1-3$ kpc. We also show the Simpson et al. (2015) FWHM observational estimate for the ALESS sample, which should be compared to the median of the ALESS-like SHARK sample (dot-dashed line in the middle, left panel of Fig. 10). The ALESS-like SHARK sample is slightly more extended than the $S_{\text {band-7 }}>1 \mathrm{mJy}$, reflecting the weak correlation we find between the median galaxy size and the band- 7 flux. Within the uncertainties, we find SHARK to agree well with Simpson et al. (2015).

\subsection{The molecular gas emission of SMGs}

The bottom panels of Fig. 10 shows the $\mathrm{CO}(1-0)$ brightness luminosity as a function of redshift, $L_{\mathrm{CO}(1-0)}^{\prime}$, for three samples selected in bands 7, 6 and 4. We find that higher fluxes are associated with higher $L_{\mathrm{CO}(1-0)}^{\prime}$, and that at fixed flux, galaxies selected in band 4 have higher $L_{\mathrm{CO}(1-0)}^{\prime}$ than those selected in band 6 and 7 . For reference we also show the predicted $L_{\mathrm{CO}(1-0)}^{\prime}$ of MS galaxies with stellar masses $10^{9}-10^{10} \mathrm{M}_{\odot}$ and find the samples with $S>1 \mathrm{mJy}$ in bands 7,6 and 4 and the $S_{\text {band-4 }}>0.1 \mathrm{mJy}$ sample to be $\mathrm{CO}(1-0)$ brighter. However, note that the samples with $S>1$ mJy in bands 7 and 4 are only $\approx 0.3-0.5$ dex above the median $L_{\mathrm{CO}(1-0)}^{\prime}$ of MS galaxies, while galaxies with $S_{\text {band-4 }}>1$ mJy are $\approx 1.2$ dex above MS galaxies. Some of these differences come from the different stellar masses being probed by the samples (see top panels in Fig. 8), given that $L_{\mathrm{CO}(1-0)}^{\prime}$ increases with increasing stellar mass, on average, and some of this is due to the complex scaling between $L_{\mathrm{CO}(1-0)}^{\prime}$ and SFR at fixed stellar mass presented in Fig. A3.

Bothwell et al. (2013) presented one of the most comprehensive studies of the CO SLED of SMGs in the literature, which has been extended to brighter (Spilker et al. 2014; Yang et al. 2017; Cañameras et al. 2018) and fainter (Valentino et al. 2020a) objects. In order to compare with Bothwell et al. (2013), we select galaxies in SHARK to follow the same $850 \mu \mathrm{m}$ continuum emission of the sample in Bothwell et al. (2013) as it was done with the ALESS-like sample of Fig. 7. The median redshift of this SHARK Bothwell13-like sample is 2.15, while for Bothwell et al. (2013) this is 2.28, in excellent agreement.

From the Bothwell13-like sample, we compute the median CO SLED with the corresponding $16^{\text {th }}-84^{\text {th }}$ percentiles 


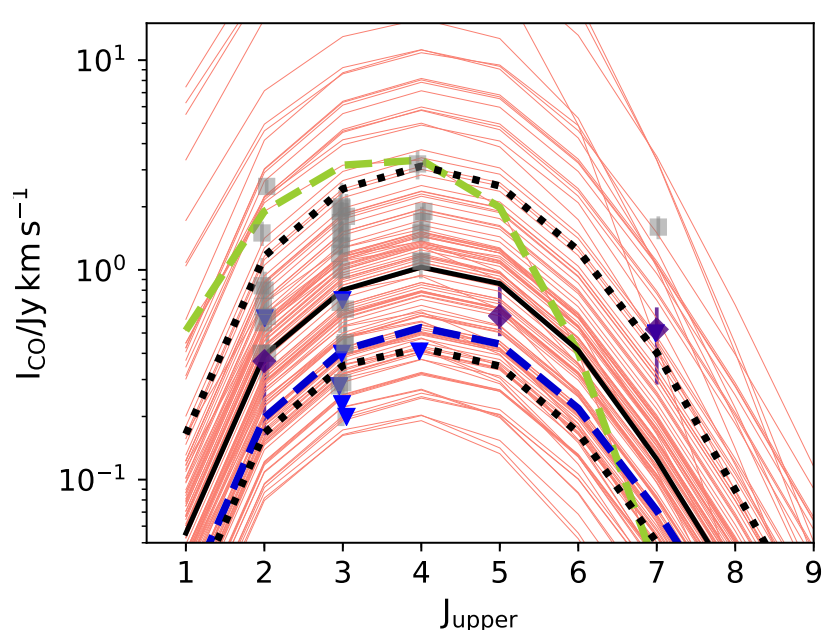

Figure 11. CO SLED for SMGs selected in SHARK to follow the $850 \mu \mathrm{m}$ flux distribution of the SMG sample of Bothwell et al. (2013). Thin lines show individual SHARK-selected galaxies, while the thick solid and dotted lines show the median and the $1 \sigma$ percentile ranges. The green and blue dashed lines show examples of SMGs with very weak and strong AGN hard X-ray emission, respectively. Individual detections from Bothwell et al. (2013) are shown as squares, while upper limits are shown as down-pointing triangles. The $\mathrm{x}$-axis position of observations are slightly perturbed to aid visualization. We also show the median CO SLED for galaxies with $L_{\mathrm{IR}}>10^{12} \mathrm{~L}_{\odot}$ above the MS by a factor $\geq 3.5$, equivalent to the galaxies in Bothwell et al. (2013), presented in Valentino et al. (2020a) (diamonds), which we place at $z=2$ for comparison with SHARK and Bothwell et al. (2013) (their original median redshift is $z=1.3$ ).

ranges and compare with the observations in Fig. 11. Fig. 11 also shows the individual SHARK galaxies in the Bothwell13like sample. SHARK appears to be systematically fainter than the Bothwell et al. (2013) observations, with detectiosn falling preferentially between the median and the $84^{\text {th }}$ percentile of SHARK galaxies.

Note that although most CO SLEDs in Fig. 11 peak at around $J_{\text {upper }}=4-5$, there is great diversity in their shapes. This is most noticeable at $J_{\text {upper }} \geq 5$, where some galaxies experience a sharp decrease in the CO intensity, while others display a gentle decrease. The latter is driven by the effect of AGN in those SMGs, modelled by the inclusion of the hard X-ray flux (see $\S 2.4$ ), which tends to boost the line intensity of high $\mathrm{CO}$ transitions. An example of this is visualised in Fig. 11, where we highlight two SMGs with a low $\left(\approx 10^{38} \mathrm{erg} \mathrm{s}^{-1}\right)$ and high $\left(\approx 2 \times 10^{43} \mathrm{erg} \mathrm{s}^{-1}\right)$ AGN hard X-ray luminosity. The former displays a sharp $I_{\mathrm{CO}}$ decrease at $J_{\text {upper }}>4$, while the latter displays a gentle decay. The reason why the X-ray bright galaxy has a lower $I_{\mathrm{CO}}$ at $J_{\text {upper }}<5$ is because it is at higher redshift, $z \approx 2.3$, compared to the faint one, $z \approx 1.7$.

To compare SHARK with a more representative median CO SLED of bright starburst galaxies, we show in Fig. 11 the measurements of Valentino et al. (2020a) for starburst galaxies with $L_{\mathrm{IR}}>10^{12} \mathrm{~L}_{\odot}$ that are above the MS by factors $\geq 3.5$, similar to the galaxies in Bothwell et al. (2013). We find the SHARK $I_{\mathrm{CO}}$ medians to be in excellent agreement with Valentino et al. (2020a) at transitions $J_{\text {upper }}=2,5$, but are too faint at $J_{\text {upper }}=7$. This shows that despite the CO SLED modelling included in SHARK accounting for an Xray like source, its effect is likely insufficient to explain the high excitation at high $J_{\text {upper }}$. Inclusion of additional heating sources, such as shocks, could increase the excitation at higher $J_{\text {upper }}$.

It is worth noting that the level of agreement we find with observed CO SLEDs is somewhat surprising given the assumptions we make in our modelling (see $\S 2.4$ for a discussion), and shows that some of the effects we ignore may be cancelling each other to some extent.

\section{THE CONTRIBUTION OF SMGS TO THE COSMIC STAR FORMATION RATE AND $\mathrm{H}_{2}$ DENSITY HISTORY}

Current observational estimates of the contribution of FIRselected galaxies to the CSFRD of the Universe are sparse at $z \gtrsim 4$, due to the small areas probed by deep FIR surveys (Casey et al. 2018a). The success of SHARK in reproducing a variety of observed properties of SMGs, from their opticalto-NIR emission, to their derived intrinsic properties, makes it an ideal tool to explore this question.

Fig. 12 shows the CSFRD evolution at $0 \leq z \leq 10$ and the contribution from galaxies selected in different flux ranges in ALMA bands 7,6 and 4. The total CSFRD is the same in all panels. We show observational constraints from Driver et al. (2018); Bouwens et al. (2012). For the latter we show the uncorrected and corrected measurements, which were derived from rest-frame UV LFs. From the predicted total CSFRD in SHARK we can conclude that rest-frame UV-selected galaxies after being corrected by dust attenuation, are able to recover the total CSFRD closely. Hence, in our model framework, no significant cosmic SFR would be missing, which would agree with an overall dust-poor galaxy population at $z \gtrsim 5$ (similar to "model A" in Casey et al. 2018b). This is because most galaxies in SHARK at those high redshifts are metal poor $\left(Z_{\text {gas }}<0.25 Z_{\odot}\right)$, and only a handful of galaxies at those redshifts have metallicities above that (see Fig. 14 in Lagos et al. 2019 and analysis therein).

Moving to the contribution from different ALMA band selected galaxies to the total CSFRD, we can see that galaxies with $S_{\text {band }-7}>1 \mathrm{mJy}$ contribute a constant $\approx 44 \%$ at $0 \leq z \leq 3$, after which their contribution starts to decrease. Nevertheless, $S_{\text {band-7 }}>1$ mJy galaxies should make up $>10 \%$ of the total CSFRD out to $z \approx 5$, well within the "cosmic dawn" regime. Going fainter to $0.1<S_{\text {band }-7} /$ mJy $<1$, we see that these galaxies contribute $>10 \%$ at $z \lesssim 8$. Fainter galaxies, however, dominate at $z \gtrsim 6$. SHARK galaxies selected in ALMA band 6 behave similarly, though the contribution of galaxies with $S_{\text {band-6 }}>1$ mJy never exceed $\approx 28 \%$. Band 4 selected galaxies behave quite differently, with $S_{\text {band-4 }}>1 \mathrm{mJy}$ making a negligible contribution to the CSFRD at all redshifts. However, going to galaxies with $0.1<S_{\text {band-4 }} / \mathrm{mJy}<1$, we find that they make up $>10 \%$ of the CSFRD at $z<6.8$. This means that $2 \mathrm{~mm}$ selected surveys should aim to go down to fluxes of $0.1 \mathrm{mJy}$ if the aim is to constrain the contribution of dust galaxies to the CSFRD.

Another quantity that has sparked significant attention with the advent of ALMA is the cosmic molecular gas den- 

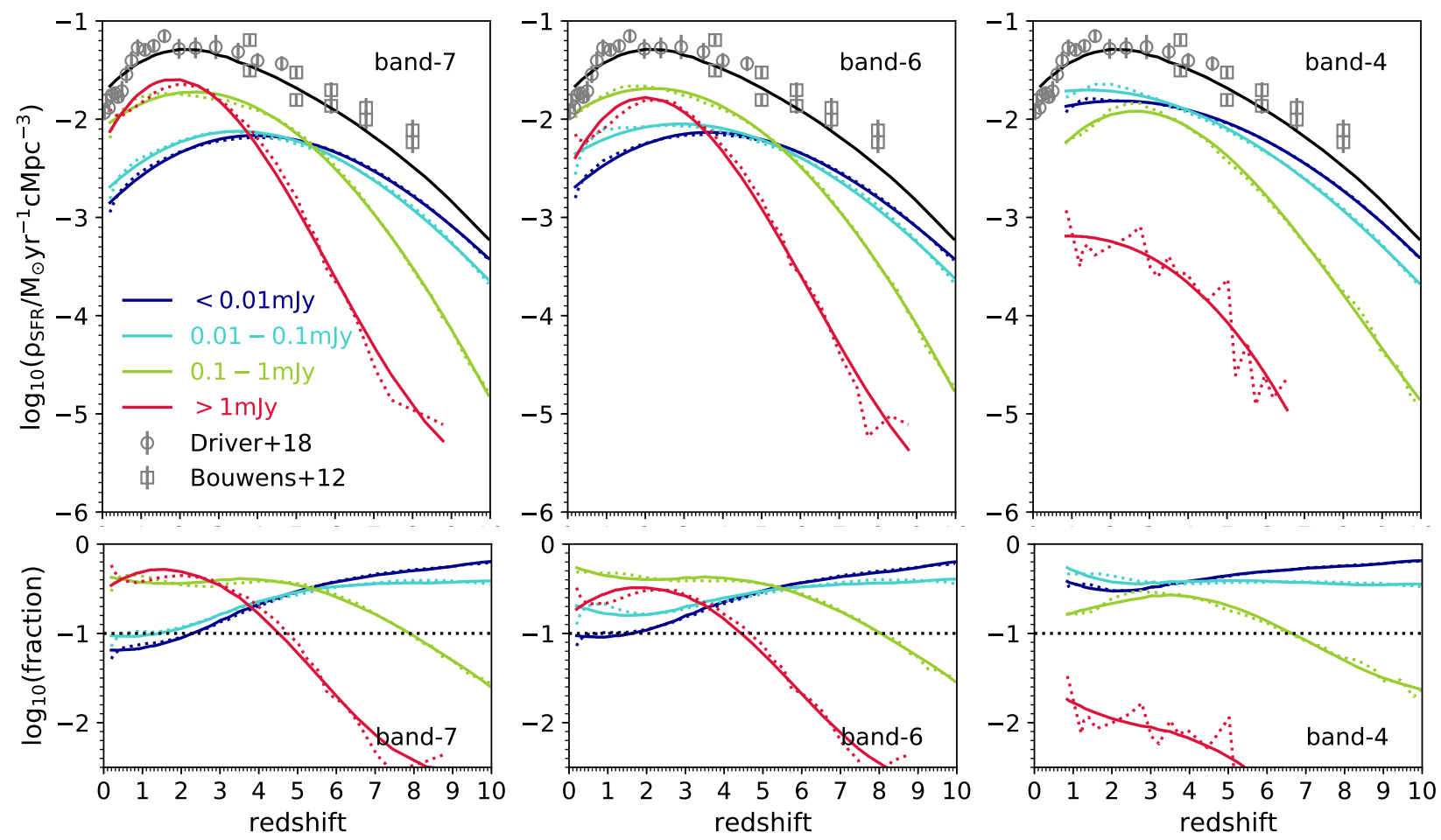

Figure 12. Top panel: Cosmic SFR of the universe. The black line shows the predicted total cosmic SFR, while data points show the observational estimates of Driver et al. (2018) and Bouwens et al. (2012). For the latter we show both the corrected and uncorrected measurements, which were derived from UV LFs. The colour dotted lines show the predicted SFR density contribution of galaxies selected in ALMA band-7 (left panel), band-6 (middle panel) and band-4 (right panel) selected galaxies in 4 different flux bins. The coloured solid lines show a spline fit to the latter, which are presented in Table B1. Bottom panel: The fractional contribution from the samples of the top panel to the total SFR density predicted by SHARK. For reference, the horizontal dotted line shows a contribution of $10 \%$.

sity, $\rho_{\mathrm{H}_{2}}$, evolution. Recent constraints from the ASPECS survey (Decarli et al. 2019), COLDz (Riechers et al. 2019) and ALMACAL-CO (Hamanowicz et al. in preparation) suggest $\rho_{\mathrm{H}_{2}}$ increases from $z=0$ to $z \approx 1.5-3.5$ followed by a decline towards higher redshifts. This is qualitatively similar to the CSFRD, however, the exact redshift peak of $\rho_{\mathrm{H}_{2}}$ and the magnitude of its evolution are still highly uncertain. We use SHARK to investigate the contribution from the flux selected samples above to $\rho_{\mathrm{H}_{2}}$ as a function of redshift in Fig. 13. First, comparing the total $\rho_{\mathrm{H}_{2}}$ to observations, we find our measurements agree very well with the measurements within the uncertainties, except for the ASPECS $\rho_{\mathrm{H}_{2}}$ at $z \approx 1.5$. There is a clear tension between the ASPECS and the COLDz plus ALMACAL-CO measurements at $z \approx 1.5-2$, which likely comes from the underlying assumed CO SLED of the surveys being different. Riechers et al. (2020) argued that ASPECS assumes a CO SLED that were too low excitation based on new measurements of $\mathrm{CO}(1-0)$. If that is the case, then the Decarli et al. (2019) measurements are overestimated by a factor of $\approx 2$. The tension between ASPECS and other $z \approx 0$ measurements on the other hand is most likely due to the small area probed by ASPECS $\left(0.0013 \mathrm{deg}^{2}\right)$, which implies a cosmic variance of $\approx 50 \%(78 \%)$ at $0<z<0.5(0<z<0.25)$ according to the cosmic variance calculator of Driver \& Robotham (2010). This shows that the errorbars reported in observations are likely underestimated as they do not include systematic effects such as the ones mentioned here.

Regarding the contribution of the different ALMA band flux selected samples, we generally find that bright ALMA bands 7, 6 and 4 galaxies, $S>1 \mathrm{mJy}$, contribute less to $\rho_{\mathrm{H}_{2}}$ than their contribution to the CSFRD. The maximal contribution of $S_{\text {band }-7}>1 \mathrm{mJy}$ galaxies to $\rho_{\mathrm{H}_{2}}$ is $\approx 30 \%$ at $z \approx 0$, decreasing to $<10 \%$ at $z \gtrsim 3.2$. In the case of $S_{\text {band-6 }}>1$ mJy galaxies, they make up $\approx 8-10 \%$ of $\rho_{\mathrm{H}_{2}}$ at $0 \lesssim z \lesssim 3.5$, percentage that decreases steeply at higher redshifts. $S_{\text {band }-4}>1 \mathrm{mJy}$ galaxies have a negligible contribution to $\rho_{\mathrm{H}_{2}}, \ll 1 \%$, throughout the whole redshift range.

Moving to the samples with $0.1<S /$ mJy $<1$, we find them to be the dominant source of $\mathrm{H}_{2}$ at $z \leqslant 3$ for band 7 and 6 . However, at band 4, those galaxies still make up $<10 \%$ of $\rho_{\mathrm{H}_{2}}$ at all redshifts. It is interesting to see that the fainter sample, $0.01<S / \mathrm{mJy}<0.1$ is not dominant at any redshifts in bands 7 and 6 , and at the redshift in which $0.1<S / \mathrm{mJy}<1$ galaxies cease to be dominant $(z \approx 3)$, the majority of $\mathrm{H}_{2}$ starts to come from the faintest galaxies $S<0.01 \mathrm{mJy}$. In band 4 , we see that galaxies with $S<$ $0.01 \mathrm{mJy}$ and $0.01<S / \mathrm{mJy}<0.1$ contribute similarly to $\rho_{\mathrm{H}_{2}}$ at $z \lesssim 1$, but at higher redshift the vast majority of $\mathrm{H}_{2}$ is contributed by galaxies with $S<0.01 \mathrm{mJy}$.

We do not investigate the equivalent atomic hydrogen density here as previous galaxy formation simulations have 

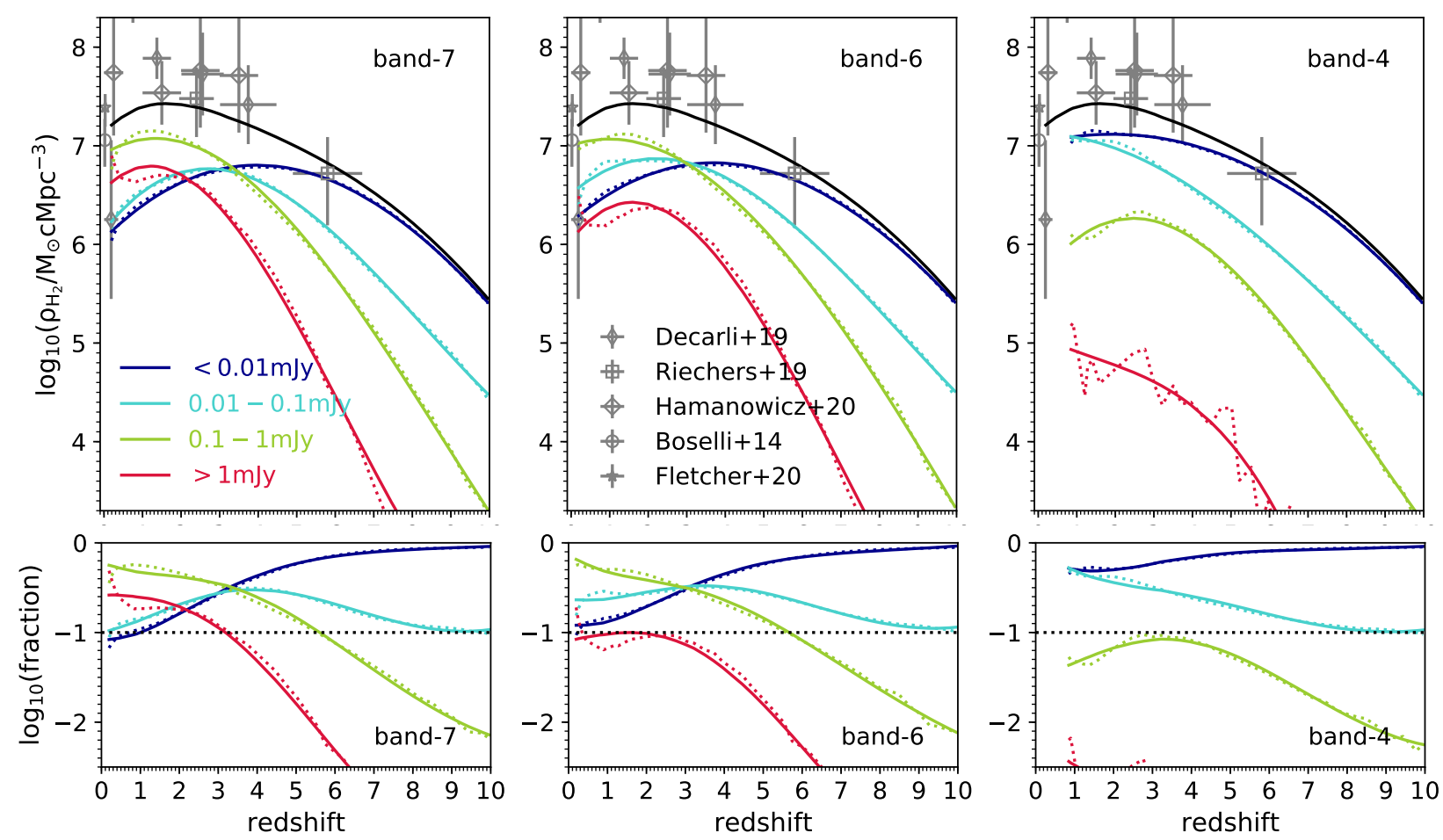

Figure 13. As in Fig. 12 but for the cosmic $\mathrm{H}_{2}$ density evolution of the universe. Observational estimates shown here correspond to Boselli et al. (2014); Fletcher et al. (2020); Decarli et al. (2019); Riechers et al. (2019); Hamanowicz et al. (in preparation), as labelled in the middle panel.

shown this to be dominated by lower stellar mass galaxies, with a negligible contribution from massive galaxies, $>10^{10} \mathrm{M}_{\odot}$ (except at low redshift, $z \lesssim 0.2$; e.g. Davé et al. 2013; Lagos et al. 2014). The latter are the typical masses we find in our bright $S>1 \mathrm{mJy}$ samples (see top panels in Fig. 8).

\section{DISCUSSION AND CONCLUSIONS}

In this work we present a thorough exploration of the properties of submm and mm-selected galaxies in the SHARK semi-analytic model of galaxy formation across a wide redshift range, $0 \leq z \leq 10$. To predict the broad-band SEDs of galaxies we use Viperfish (Lagos et al. 2019; Robotham et al. 2020), which uses the stellar populations of BC03 (with our assumed universal IMF of Chabrier 2003) and adopts the Charlot \& Fall (2000) attenuation parametrisation. We scale the latter parameters with the galaxy's dust surface density, following the RT analysis of the EAGLE hydrodynamical simulations of Trayford et al. (2020). Critical for this work, we re-emit the absorbed light assuming energy balance and adopting the IR templates of Dale et al. (2014). We assume two distinct power-law indices for the local interstellar radiation field of the diffuse and birth clouds dust components. This is equivalent to assuming two average dust temperatures for the diffuse and birth cloud dust components that are constant with redshift and galaxy properties. Despite the simplicity of some of these assumptions, we find excellent agreement with observations over a broad range of diagnostics. We summarise our main findings below:

- We tested the SHARK predicted number counts against measurements at $650 \mu \mathrm{m}, 870 \mu \mathrm{m}, 1.1 \mathrm{~mm}$ and $2 \mathrm{~mm}$ (all the ALMA bands that have been used to compute number counts) finding excellent agreement (Fig. 3). Significant tension is however seen at the $1 \mathrm{~mm}$ faint end between observations, and SHARK predictions fall in between these measurements, though closer to Fujimoto et al. (2016). We also compare the predicted redshift distributions of SHARK with observations at $100 \mu \mathrm{m}, 250 \mu \mathrm{m}, 450 \mu \mathrm{m}, 850 \mu \mathrm{m}, 1.1 \mathrm{~mm}$ and $2.2 \mathrm{~mm}$, finding broad agreement (Fig. 4). There is a small tendency of SHARK galaxies to be at slightly lower redshifts than some of the most recent $870 \mu \mathrm{m}$ measurements, but given the associated uncertainties of photometric redshifts it is unclear whether this tension is significant. We find that $S>1 \mathrm{mJy}$ sources are a mix of galaxy merger- and disk instabilities- driven starbursts, showing that they are an inhomogeneous population.

- We find that current deep optical surveys (e.g. HUDF and HSC) are deep enough as to detect most of the ALMA band-7 $>1$ mJy SMGs at $z<4$, but are not sensitive enough to find counterparts for all SMGs at higher redshifts (Fig. 5). In fact, in SHARK $10 \%$ of these SMGs are IRAC dark in agreement with Dudzevičiūte et al. (2020). The latter in SHARK are at significantly higher redshift, $z \approx 3.5$, compare to the IRAC bright ones, $z \approx 2.2$ (Table 1 ), and have higher rest-frame $\mathrm{V}$-band attenuation $\left(A_{\mathrm{V}} \approx 2.2 \mathrm{com}-\right.$ pared to $\left.A_{\mathrm{V}} \approx 1.5\right)$. We find that a nominal $10,000 \mathrm{~s}$ NIR 
JWST survey should be deep enough as to see all band-7 $\gtrsim 0.01 \mathrm{mJy}$. If we study galaxies selected at bands 6 and 4 we find their optical counterparts to be brighter than band 7 galaxies at fixed flux, so current optical surveys can detect those up to higher redshifts (Figs. A1 and A2). We find that about $40-50 \%$ of SHARK galaxies classified as passive in the (u-r) vs. (r-J) plane at $1.5 \leq z \leq 3.5$ are in fact star-forming with $850 \mu \mathrm{m}$ fluxes $>0.1 \mathrm{mJy}$ (Fig. 6). We compared the stacked FUV-to-FIR SED of SHARK SMGs that follow the same band 7 flux distribution as ALESS da Cunha et al. (2015) and compare with their stacked SED finding excellent agreement (Fig. 7). All this evidence shows that SHARK captures the UV-to-MIR properties of SMGs quite well.

- Regarding intrinsic properties of submm and $\mathrm{mm}$ selected SHARK galaxies, we find that galaxies with $870 \mu \mathrm{m}$ fluxes $>1 \mathrm{mJy}$ have stellar masses $\approx 10^{10}-10^{11} \mathrm{M}_{\odot}$, are mildly above the MS by factors of $\approx 2-3$, and live in intermediate mass halos $\approx 10^{12.3}-10^{13} \mathrm{M}_{\odot}$, in agreement with observations (Fig. 8). These properties are similar to those found in the EAGLE hydrodynamical simulations for similarly bright SMGs at $1 \leq z \leq 3$ (McAlpine et al. 2019). Interestingly, we find that band 4 galaxies with fluxes $>1 \mathrm{mJy}$ at $z \gtrsim 4$ in SHARK trace the most massive galaxies in the simulation, $M_{\star} \gtrsim 10^{11} \mathrm{M}_{\odot}$, have sSFRs above the MS by factors of $\gtrsim 7-10$ and live in the most massive haloes at those redshifts. This makes them ideal tracers of protoclusters. We also find that the vast majority of bright SMGs in bands 7, 6 and 4 in SHARK are experiencing starbursts, but the mechanism behind comes in two very different flavours: galaxy mergers and disk instabilities, almost at the $50-50$ level (Fig. 10). We also find SMGs to have SFR-weighted effective radii $\approx 1-5 \mathrm{kpc}$, with a significant redshift dependence, in a way that high-z SMGs are more compact than low-z SMGs. We also find a very weak positive correlation between the flux and the spatial extent of galaxies at fixed redshift, in agreement with the observational derivations of Fujimoto et al. (2017).

- The dust masses and temperatures of SHARK galaxies with $870 \mu \mathrm{m}$ fluxes $>1 \mathrm{mJy}$ span a wide range $10^{7}-10^{9} \mathrm{M}_{\odot}$ and $37-45 \mathrm{~K}$, respectively (Fig. 9). These values agree with the observations within the (large) uncertainties. We find that galaxies with $2 \mathrm{~mm}$ fluxes $>1 \mathrm{mJy}$ tend to be more dust rich by 0.5 dex and have hotter dust temperatures $T_{\text {dust }} \approx 42-45 \mathrm{~K}$ than the above sample. We also find that the rest frame $\mathrm{V}$-band attenuation increases with increasing redshift at fixed flux, and for SHARK galaxies with $870 \mu \mathrm{m}$ fluxes $>1$ mJy the median goes from $A_{\mathrm{V}} \approx 1.2$ at $z \approx 1$ to $A_{\mathrm{V}} \approx 2$ at $z \approx 4$. These $A_{\mathrm{V}}$ values again agree well with observations within the uncertainties. SHARK galaxies selected at longer wavelengths tend to display higher $A_{\mathrm{V}}$ at fixed flux and redshift. In fact, galaxies with $2 \mathrm{~mm}$ fluxes $>1$ mJy have $A_{\mathrm{V}} \gtrsim 1.8$ at all redshifts (Fig. 9).

- We study the CO emission of submm and mm-selected galaxies and find that the $\mathrm{CO}(1-0)$ luminosity is relatively constant with redshift at fixed flux. Galaxies with $870 \mu \mathrm{m}$, $1.1 \mathrm{~mm}$ and $2 \mathrm{~mm}$ fluxes $>1 \mathrm{mJy}$ have average $L_{\mathrm{CO}(1-0)}^{\prime} \approx$ $10^{9.5}, 10^{9.7}$ and $10^{10.5} \mathrm{~K} \mathrm{~km} \mathrm{~s}^{-1} \mathrm{pc}^{2}$, respectively (Fig. 10). We build the CO SLED of $850 \mu \mathrm{m}$ SMGs selected to have the same flux distribution as Bothwell et al. (2013) and compare to their CO SLEDs. We find that the CO SLED shape resembles the observations, displaying a peak at $J_{\text {upper }} \approx 4$, but the normalisation appears to be $\approx 1.5$ times lower in SHARK
(Fig. 11). Compared to the median CO SLEDs of starburst galaxies with $L_{\mathrm{IR}}>10^{12} \mathrm{~L}_{\odot}$ of Valentino et al. (2020a), we find SHARK is in excellent agreement at $J_{\text {upper }} \leq 5$, but is too faint by $\approx 0.2 \mathrm{Jy} \mathrm{km} \mathrm{s}^{-1}$ at $J_{\text {upper }}=7$. The CO SLED modelling of SHARK includes an X-ray like source (powered by AGN) in addition to the traditional PDR. Our comparison with observations suggests that additional excitation sources, such as shocks, may be necessary.

- Finally, we quantify the contribution of bright $870 \mu \mathrm{m}$ and $1.1 \mathrm{~mm}$-selected galaxies ( $>1 \mathrm{mJy}$ ) to the CSFRD (Fig. 12), finding that they make a significant contribution $(>10 \%)$ at $z \approx 4-5$, but become negligible at higher redshifts. In contrast, $2 \mathrm{~mm}$ bright galaxies ( $>1 \mathrm{mJy}$ ) make a small contribution, $\lesssim 2 \%$, at all redshifts. Our predictions are that dusty galaxies make a negligible contribution to the CSFRD at $z \gtrsim 4$. We extend this analysis to $\rho_{\mathrm{H}_{2}}$ (Fig. 13) and find that the contribution of these bright submm and $\mathrm{mm}$ galaxies ( $>1 \mathrm{mJy}$ ) is even smaller, with $870 \mu \mathrm{m}$ and $1.1 \mathrm{~mm}$-selected galaxies contributing $\gtrsim 10 \%$ at $z \lesssim 3$ and $\lesssim 2.5$, respectively.

The broad agreement we find between the predicted properties of submm and mm-selected galaxies in SHARK with several observations are very encouraging, and make SHARK an ideal tool to plan survey strategies to follow up SMGs with different current and upcoming telescopes, and to make predictions about what future surveys may see (which we do here). It also shows that the adopted assumptions in the model are at least appropriate to aid this success, despite their simplicity. In the future we will explore physical ways of scaling the dust temperature and radiation field spectrum with local galaxy properties, aided by recent progress in detailed RT analysis of galaxies in hydrodynamical simulations (e.g. Trayford et al. 2020; Lovell et al. 2020); and will test the systematic uncertainties of FIR SED fitting to derive dust parameters, such as temperature and mass.

\section{ACKNOWLEDGEMENTS}

We thank Chris Lovell, Paul van de Werf, Jacqueline Hogde, Matus Reykn, Joop Schaye, Desika Narayanan and Ian Smail for useful discussions throughout the writing of this manuscript. We also thank the anonymous referee for their insightful report. CL and $\mathrm{EdC}$ have received funding from the ARC Centre of Excellence for All Sky Astrophysics in 3 Dimensions (ASTRO 3D), through project number CE170100013. CL also thanks the MERAC Foundation for a Postdoctoral Research Award. FV acknowledges support from the Carlsberg Foundation Research Grant CF18-0388 "Galaxies: Rise and Death". GEM acknowledges the Villum Fonden research grant 13160 "Gas to stars, stars to dust: tracing star formation across cosmic time" and the Cosmic Dawn Center of Excellence funded by the Danish National Research Foundation under the grant No. 140. This work was supported by resources provided by The Pawsey Supercomputing Centre with funding from the Australian Government and the Government of Western Australia. 


\section{DATA AVAILABILITY}

The lightcone used for this paper is available upon request (please email first author to get access). The SURFS simulation used in this work can be accessed freely from https:// tinyurl.com/y6ql46d4. Shark, Viperfish, and Stingray are all public codes (see $\S 2$ and 3 ), and the python scripts used to produce the plots in this paper can be found at https://github.com/cdplagos/lightcone-analysis.

\section{REFERENCES}

Aihara H., AlSayyad Y., Ando M., Armstrong R., Bosch J., Egami E., Furusawa H., Furusawa J. et al, 2019, PASJ, 71, 114

Amarantidis S., Afonso J., Messias H., Henriques B., Griffin A., Lacey C., Lagos C. d. P., Gonzalez-Perez V. et al, 2019, MNRAS, 485, 2694

Barro G., Kriek M., Pérez-González P. G., Trump J. R., Koo D. C., Faber S. M., Dekel A., Primack J. R. et al, 2016, ApJ, 827, L32

Battisti A. J., da Cunha E., Grasha K., Salvato M., Daddi E., Davies L., Jin S., Liu D. et al, 2019, ApJ, 882, 61

Baugh C. M., Lacey C. G., Frenk C. S., Granato G. L., Silva L., Bressan A., Benson A. J., Cole S., 2005, MNRAS, 356, 1191

Bayet E., Williams D. A., Hartquist T. W., Viti S., 2011, MNRAS, 414, 1583

Beckwith S. V. W., Stiavelli M., Koekemoer A. M., Caldwell J. A. R., Ferguson H. C., Hook R., Lucas R. A., Bergeron L. E. et al, 2006, AJ, 132, 1729

Berta S., Magnelli B., Nordon R., Lutz D., Wuyts S., Altieri B., Andreani P., Aussel H. et al, 2011, A\&A, 532, A49

Béthermin M., Daddi E., Magdis G., Lagos C., Sargent M., Albrecht M., Aussel H., Bertoldi F. et al, 2015, A\&A, 573, A113

Bethermin M., Fudamoto Y., Ginolfi M., Loiacono F., Khusanova Y., Capak P. L., Cassata P., Faisst A. et al, 2020, arXiv eprints, arXiv:2002.00962

Béthermin M., Le Floc'h E., Ilbert O., Conley A., Lagache G., Amblard A., Arumugam V., Aussel H. et al, 2012, A\&A, 542, A58

Bigiel F., Leroy A., Walter F., Blitz L., Brinks E., de Blok W. J. G., Madore B., 2010, AJ, 140, 1194

Bonatto C., Bica E., 2011, MNRAS, 415, 2827

Boselli A., Cortese L., Boquien M., Boissier S., Catinella B., Gavazzi G., Lagos C., Saintonge A., 2014, A\&A, 564, A67

Bothwell M. S., Smail I., Chapman S. C., Genzel R., Ivison R. J., Tacconi L. J., Alaghband -Zadeh S., Bertoldi F. et al, 2013, MNRAS, 429, 3047

Bournaud F., Chapon D., Teyssier R., Powell L. C., Elmegreen B. G., Elmegreen D. M., Duc P.-A., Contini T. et al, 2011, ApJ, 730, 4

Bournaud F., Elmegreen B. G., Teyssier R., Block D. L., Puerari I., 2010, MNRAS, 409, 1088

Bouwens R. J., Illingworth G. D., Oesch P. A., Franx M., Labbé I., Trenti M., van Dokkum P., Carollo C. M. et al, 2012, ApJ, 754,83

Brammer G. B., Whitaker K. E., van Dokkum P. G., Marchesini D., Franx M., Kriek M., Labbé I., Lee K. S. et al, 2011, ApJ, 739,24

Bravo M., Lagos C. d. P., Robotham A. S. G., Bellstedt S., Obreschkow D., 2020, arXiv e-prints, arXiv:2003.11258

Brisbin D., Miettinen O., Aravena M., Smolčić V., Delvecchio I., Jiang C., Magnelli B., Albrecht M. et al, 2017, A\&A, 608, A15

Bruzual G., Charlot S., 2003, MNRAS, 344, 1000

Cañameras R., Yang C., Nesvadba N. P. H., Beelen A., Kneissl R., Koenig S., Le Floc'h E., Limousin M. et al, 2018, A\&A, $620, \mathrm{~A} 61$
Cañas R., Elahi P. J., Welker C., del P Lagos C., Power C., Dubois Y., Pichon C., 2019, MNRAS, 482, 2039

Cai Z.-Y., Lapi A., Xia J.-Q., De Zotti G., Negrello M., Gruppioni C., Rigby E., Castex G. et al, 2013, ApJ, 768, 21

Camps P., Baes M., 2015, Astronomy and Computing, 9, 20

Camps P., Trčka A., Trayford J., Baes M., Theuns T., Crain R. A., McAlpine S., Schaller M. et al, 2018, ApJS, 234, 20

Capak P., Aussel H., Ajiki M., McCracken H. J., Mobasher B., Scoville N., Shopbell P., Taniguchi Y. et al, 2007, ApJS, 172, 99

Casey C. M., Berta S., Béthermin M., Bock J., Bridge C., Budynkiewicz J., Burgarella D., Chapin E. et al, 2012, ApJ, 761, 140

Casey C. M., Chen C.-C., Cowie L. L., Barger A. J., Capak P., Ilbert O., Koss M., Lee N. et al, 2013, MNRAS, 436, 1919

Casey C. M., Hodge J., Zavala J. A., Spilker J., da Cunha E., Staguhn J., Finkelstein S. L., Drew P., 2018a, ApJ, 862, 78

Casey C. M., Narayanan D., Cooray A., 2014, Phys. Rep., 541, 45

Casey C. M., Zavala J. A., Aravena M., Béthermin M., Caputi K. I., Champagne J. B., Clements D. L., da Cunha E. et al, 2019, ApJ, 887, 55

Casey C. M., Zavala J. A., Spilker J., da Cunha E., Hodge J., Hung C.-L., Staguhn J., Finkelstein S. L. et al, 2018b, ApJ, 862,77

Chabrier G., 2003, PASP, 115, 763

Chang R.-X., Shu C.-G., Hou J.-L., 2002, Chin. Journ. Astronomy \& Astrophysics, 2, 226

Chapman S. C., Blain A. W., Smail I., Ivison R. J., 2005, ApJ, 622,772

Charlot S., Fall S. M., 2000, ApJ, 539, 718

Chauhan G., Lagos C. D. P., Obreschkow D., Power C., Oman K., Elahi P. J., 2019, arXiv:1906.06130, arXiv:1906.06130

Chauhan G., Lagos C. d. P., Stevens A. R. H., Obreschkow D., Power C., Meyer M., 2020, arXiv e-prints, arXiv:2006.12102

Cortzen I., Magdis G. E., Valentino F., Daddi E., Liu D., Rigopoulou D., Sargent M., Riechers D. et al, 2020, A\&A, 634, L14

Cowie L. L., González-López J., Barger A. J., Bauer F. E., Hsu L. Y., Wang W. H., 2018, ApJ, 865, 106

Cowley W. I., Béthermin M., Lagos C. d. P., Lacey C. G., Baugh C. M., Cole S., 2017a, MNRAS, 467, 1231

Cowley W. I., Lacey C. G., Baugh C. M., Cole S., 2015, MNRAS, 446,1784

Cowley W. I., Lacey C. G., Baugh C. M., Cole S., Frenk C. S., Lagos C. d. P., 2019, MNRAS, 487, 3082

Cowley W. I., Lacey C. G., Baugh C. M., Cole S., Wilkinson A., 2017b, MNRAS, 469, 3396

Crain R. A., Schaye J., Bower R. G., Furlong M., Schaller M., Theuns T., Dalla Vecchia C., Frenk C. S. et al, 2015, MNRAS, 450, 1937

da Cunha E., Charlot S., Elbaz D., 2008, MNRAS, 388, 1595

da Cunha E., Charmandaris V., Díaz-Santos T., Armus L., Marshall J. A., Elbaz D., 2010, A\&A, 523, A78

da Cunha E., Groves B., Walter F., Decarli R., Weiss A., Bertoldi F., Carilli C., Daddi E. et al, 2013, ApJ, 766, 13

da Cunha E., Walter F., Smail I. R., Swinbank A. M., Simpson J. M., Decarli R., Hodge J. A., Weiss A. et al, 2015, ApJ, 806, 110

Daddi E., Cimatti A., Renzini A., Fontana A., Mignoli M., Pozzetti L., Tozzi P., Zamorani G., 2004, ApJ, 617, 746

Dale D. A., Helou G., Magdis G. E., Armus L., Díaz-Santos T., Shi Y., 2014, ApJ, 784, 83

Davé R., Anglés-Alcázar D., Narayanan D., Li Q., Rafieferantsoa M. H., Appleby S., 2019, MNRAS, 486, 2827

Davé R., Katz N., Oppenheimer B. D., Kollmeier J. A., Weinberg D. H., 2013, ArXiv:1302.3631

Davies L. J. M., Lagos C. d. P., Katsianis A., Robotham A. S. G., 
Cortese L., Driver S. P., Bremer M. N., Brown M. J. I. et al, 2019, MNRAS, 483, 1881

Decarli R., Walter F., Gónzalez-López J., Aravena M., Boogaard L., Carilli C., Cox P., Daddi E. et al, 2019, ApJ, 882, 138

Driver S. P., Andrews S. K., da Cunha E., Davies L. J., Lagos C., Robotham A. S. G., Vinsen K., Wright A. H. et al, 2018, MNRAS, 475, 2891

Driver S. P., Norberg P., Baldry I. K., Bamford S. P., Hopkins A. M., Liske J., Loveday J., Peacock J. A. et al, 2009, Astronomy and Geophysics, 50, 050000

Driver S. P., Robotham A. S. G., 2010, MNRAS, 407, 2131

Dudzevičiūtè U., Smail I., Swinbank A. M., Stach S. M., Almaini O., da Cunha E., An F. X., Arumugam V. et al, 2020, MNRAS, 494, 3828

Elahi P. J., Cañas R., Poulton R. J. J., Tobar R. J., Willis J. S., Lagos C. d. P., Power C., Robotham A. S. G., 2019a, Publications of the Astronomical Society of Australia, 36, e021

Elahi P. J., Poulton R. J. J., Tobar R. J., Cañas R., Lagos C. d. P., Power C., Robotham A. S. G., 2019b, Publications of the Astronomical Society of Australia, 36, e028

Elahi P. J., Power C., Lagos C. d. P., Poulton R., Robotham A. S. G., 2018a, Monthly Notices of the Royal Astronomical Society, 477, 616

Elahi P. J., Welker C., Power C., Lagos C. d. P., Robotham A. S. G., Cañas R., Poulton R., 2018b, MNRAS, 475, 5338

Elbaz D., Hwang H. S., Magnelli B., Daddi E., Aussel H., Altieri B., Amblard A., Andreani P. et al, 2010, A\&A, 518, L29

Elbaz D., Leiton R., Nagar N., Okumura K., Franco M., Schreiber C., Pannella M., Wang T. et al, 2018, A\&A, 616, A110

Fletcher T. J., Saintonge A., Soares P. S., Pontzen A., 2020, arXiv e-prints, arXiv:2002.04959

Fujimoto S., Ouchi M., Ono Y., Shibuya T., Ishigaki M., Nagai H., Momose R., 2016, ApJS, 222, 1

Fujimoto S., Ouchi M., Shibuya T., Nagai H., 2017, ApJ, 850, 83

Geach J. E., Chapin E. L., Coppin K. E. K., Dunlop J. S., Halpern M., Smail I., van der Werf P., Serjeant S. et al, 2013, MNRAS, 432, 53

Genzel R., Tacconi L. J., Lutz D., Saintonge A., Berta S., Magnelli B., Combes F., García-Burillo S. et al, 2015, ApJ, 800, 20

Gómez-Guijarro C., Magdis G. E., Valentino F., Toft S., Man A. W. S., Ivison R. J., Tisanić K., van der Vlugt D. et al, 2019, ApJ, 886, 88

González-López J., Novak M., Decarli R., Walter F., Aravena M., Boogaard L., Popping G., Weiss A. et al, 2020, arXiv e-prints, arXiv:2002.07199

Granato G. L., Lacey C. G., Silva L., Bressan A., Baugh C. M., Cole S., Frenk C. S., 2000, ApJ, 542, 710

Gullberg B., Smail I., Swinbank A. M., Dudzevičiūtè U., Stach S. M., Thomson A. P., Almaini O., Chen C. C. et al, 2019, MNRAS, 490, 4956

Hatsukade B., Kohno K., Yamaguchi Y., Umehata H., Ao Y., Aretxaga I., Caputi K. I., Dunlop J. S. et al, 2018, PASJ, 70, 105

Hayward C. C., Kereš D., Jonsson P., Narayanan D., Cox T. J., Hernquist L., 2011, ApJ, 743, 159

Hayward C. C., Narayanan D., Kereš D., Jonsson P., Hopkins P. F., Cox T. J., Hernquist L., 2013, MNRAS, 428, 2529

Hickox R. C., Wardlow J. L., Smail I., Myers A. D., Alexander D. M., Swinbank A. M., Danielson A. L. R., Stott J. P. et al, 2012, MNRAS, 421, 284

Hodge J. A., da Cunha E., 2020, arXiv e-prints, arXiv:2004.00934

Hodge J. A., Smail I., Walter F., da Cunha E., Swinbank A. M., Rybak M., Venemans B., Brand t W. N. et al, 2019, ApJ, 876, 130

Hodge J. A., Swinbank A. M., Simpson J. M., Smail I., Walter F., Alexander D. M., Bertoldi F., Biggs A. D. et al, 2016, ApJ, 833, 103

Ikarashi S., Ivison R. J., Caputi K. I., Aretxaga I., Dunlop J. S.,
Hatsukade B., Hughes D. H., Iono D. et al, 2015, ApJ, 810, 133

Jin S., Daddi E., Magdis G. E., Liu D., Schinnerer E., Papadopoulos P. P., Gu Q., Gao Y. et al, 2019, ApJ, 887, 144

Karim A., Swinbank A. M., Hodge J. A., Smail I. R., Walter F., Biggs A. D., Simpson J. M., Danielson A. L. R. et al, 2013, MNRAS, 432, 2

Klitsch A., Zwaan M. A., Smail I., Peroux C., Biggs A. D., Chen C.-C., Ivison R. J., Popping G. et al, 2020, arXiv e-prints, arXiv:2005.01733

Krumholz M. R., McKee C. F., Tumlinson J., 2009, ApJ, 699, 850 Lacey C. G., Baugh C. M., Frenk C. S., Benson A. J., Bower R. G., Cole S., Gonzalez-Perez V., Helly J. C. et al, 2016, MNRAS, 462, 3854

Lagos C. D. P., Baugh C. M., Zwaan M. A., Lacey C. G., Gonzalez-Perez V., Power C., Swinbank A. M., van Kampen E., 2014, MNRAS, 440, 920

Lagos C. d. P., Bayet E., Baugh C. M., Lacey C. G., Bell T. A., Fanidakis N., Geach J. E., 2012, MNRAS, 426, 2142

Lagos C. d. P., Robotham A. S. G., Trayford J. W., Tobar R., Bravo M., Bellstedt S., Davies L. J. M., Driver S. P. et al, 2019, MNRAS, 489, 4196

Lagos C. d. P., Tobar R. J., Robotham A. S. G., Obreschkow D., Mitchell P. D., Power C., Elahi P. J., 2018, MNRAS, 481, 3573

Lange R., Moffett A. J., Driver S. P., Robotham A. S. G., Lagos C. d. P., Kelvin L. S., Conselice C., Margalef-Bentabol B. et al, 2016, MNRAS, 462, 1470

Leja J., Tacchella S., Conroy C., 2019, ApJ, 880, L9

Leroy A. K., Walter F., Brinks E., Bigiel F., de Blok W. J. G., Madore B., Thornley M. D., 2008, AJ, 136, 2782

Lovell C. C., Geach J. E., Davé R., Narayanan D., Li Q., 2020, arXiv e-prints, arXiv:2006.15156

Magnelli B., Karim A., Staguhn J., Kovács A., Jiménez-Andrade E. F., Casey C. M., Zavala J. A., Schinnerer E. et al, 2019, ApJ, 877, 45

Mawatari K., Inoue A. K., Hashimoto T., Silverman J., Kajisawa M., Yamanaka S., Yamada T., Davidzon I. et al, 2020, ApJ, 889,137

McAlpine S., Helly J. C., Schaller M., Trayford J. W., Qu Y., Furlong M., Bower R. G., Crain R. A. et al, 2015, ArXiv:1510.01320

McAlpine S., Smail I., Bower R. G., Swinbank A. M., Trayford J. W., Theuns T., Baes M., Camps P. et al, 2019, MNRAS, 488,2440

McConnell N. J., Ma C.-P., 2013, ApJ, 764, 184

Michałowski M. J., Dunlop J. S., Ivison R. J., Cirasuolo M., Caputi K. I., Aretxaga I., Arumugam V., Austermann J. E. et al, 2012, MNRAS, 426, 1845

Miettinen O., Smolčić V., Novak M., Aravena M., Karim A., Masters D., Riechers D. A., Bussmann R. S. et al, 2015, A\&A, 577, A29

Muñoz Arancibia A. M., González-López J., Ibar E., Bauer F. E., Carrasco M., Laporte N., Anguita T., Aravena M. et al, 2018, A\&A, 620, A125

Muldrew S. I., Hatch N. A., Cooke E. A., 2015, MNRAS, 452, 2528

Narayanan D., Conroy C., Davé R., Johnson B. D., Popping G., 2018, ApJ, 869, 70

Narayanan D., Turk M. J., Robitaille T., Kelly A. J., Connor McClellan B., Sharma R. S., Garg P., Abruzzo M. et al, 2020, arXiv e-prints, arXiv:2006.10757

Obreschkow D., Klöckner H. R., Heywood I., Levrier F., Rawlings S., 2009, ApJ, 703, 1890

Oteo I., Zwaan M. A., Ivison R. J., Smail I., Biggs A. D., 2016, ApJ, 822, 36

-, 2017, ApJ, 837, 182

Planck Collaboration, Ade P. A. R., Aghanim N., Arnaud M., 
Ashdown M., Aumont J., Baccigalupi C., Banday A. J. et al, 2016, A\&A, 594, A13

Popping G., Pillepich A., Somerville R. S., Decarli R., Walter F., Aravena M., Carilli C., Cox P. et al, 2019, ApJ, 882, 137

Poulton R. J. J., Power C., Robotham A. S. G., Elahi P. J., 2019, MNRAS, 2798

Poulton R. J. J., Robotham A. S. G., Power C., Elahi P. J., 2018, Publications of the Astronomical Society of Australia, 35, 42

Puglisi A., Daddi E., Liu D., Bournaud F., Silverman J. D., Circosta C., Calabrò A., Aravena M. et al, 2019, ApJ, 877, L23

Rémy-Ruyer A., Madden S. C., Galliano F., Galametz M., Takeuchi T. T., Asano R. S., Zhukovska S., Lebouteiller V. et al, 2014, A\&A, 563, A31

Reuter C., Vieira J. D., Spilker J. S., Weiss A., Aravena M., Archipley M., Bethermin M., Chapman S. C. et al, 2020, arXiv e-prints, arXiv:2006.14060

Riechers D. A., Boogaard L. A., Decarli R., Gonzalez-Lopez J., Smail I., Walter F., Aravena M., Carilli C. L. et al, 2020, arXiv e-prints, arXiv:2005.09653

Riechers D. A., Pavesi R., Sharon C. E., Hodge J. A., Decarli R., Walter F., Carilli C. L., Aravena M. et al, 2019, ApJ, 872, 7

Robotham A. S. G., Bellstedt S., Lagos C. d. P., Thorne J. E., Davies L. J., Driver S. P., Bravo M., 2020, MNRAS, 495, 905

Sargent M. T., Daddi E., Béthermin M., Aussel H., Magdis G., Hwang H. S., Juneau S., Elbaz D. et al, 2014, ApJ, 793, 19

Schaye J., Crain R. A., Bower R. G., Furlong M., Schaller M., Theuns T., Dalla Vecchia C., Frenk C. S. et al, 2015, MNRAS, 446,521

Schinnerer E., Groves B., Sargent M. T., Karim A., Oesch P. A., Magnelli B., LeFevre O., Tasca L. et al, 2016, ApJ, 833, 112

Schreiber C., Elbaz D., Pannella M., Ciesla L., Wang T., Franco M., 2018, A\&A, 609, A30

Schreiber C., Pannella M., Elbaz D., Béthermin M., Inami H., Dickinson M., Magnelli B., Wang T. et al, 2015, A\&A, 575, A74

Seo W.-Y., Kim W.-T., Kwak S., Hsieh P.-Y., Han C., Hopkins P. F., 2019, ApJ, 872, 5

Simpson J. M., Smail I., Swinbank A. M., Almaini O., Blain A. W., Bremer M. N., Chapman S. C., Chen C.-C. et al, 2015, ApJ, 799, 81

Simpson J. M., Swinbank A. M., Smail I., Alexand er D. M., Brandt W. N., Bertoldi F., de Breuck C., Chapman S. C. et al, 2014, ApJ, 788, 125

Smolčić V., Aravena M., Navarrete F., Schinnerer E., Riechers D. A., Bertoldi F., Feruglio C., Finoguenov A. et al, 2012, A\&A, 548, A4

Spilker J. S., Marrone D. P., Aguirre J. E., Aravena M., Ashby M. L. N., Béthermin M., Bradford C. M., Bothwell M. S. et al, 2014, ApJ, 785, 149

Staguhn J. G., Kovács A., Arendt R. G., Benford D. J., Decarli R., Dwek E., Fixsen D. J., Hilton G. C. et al, 2014, ApJ, 790, 77

Swinbank A. M., Smail I., Longmore S., Harris A. I., Baker A. J., De Breuck C., Richard J., Edge A. C. et al, 2010, Nature, 464, 733

Toft S., Smolčić V., Magnelli B., Karim A., Zirm A., Michalowski M., Capak P., Sheth K. et al, 2014, ApJ, 782, 68

Trayford J. W., Lagos C. d. P., Robotham A. S. G., Obreschkow D., 2020, MNRAS, 491, 3937

Umehata H., Tamura Y., Kohno K., Ivison R. J., Smail I., Hatsukade B., Nakanishi K., Kato Y. et al, 2017, ApJ, 835, 98

Valentino F., Daddi E., Puglisi A., Magdis G. E., Liu D., Kokorev V., Cortzen I., Madden S. C. et al, 2020a, arXiv e-prints, arXiv:2006.12521

Valentino F., Tanaka M., Davidzon I., Toft S., Gómez-Guijarro C., Stockmann M., Onodera M., Brammer G. et al, 2020b, ApJ, 889, 93

Vazdekis A., Koleva M., Ricciardelli E., Röck B., Falcón-Barroso

\section{J., 2016, MNRAS, 463, 3409}

Veilleux S., Kim D. C., Sanders D. B., 2002, ApJS, 143, 315

Virtanen P., Gommers R., Oliphant T. E., Haberland M., Reddy T., Cournapeau D., Burovski E., Peterson P. et al, 2020, Nature Methods, 17, 261

Wardlow J. L., Smail I., Coppin K. E. K., Alexand er D. M., Brandt W. N., Danielson A. L. R., Luo B., Swinbank A. M. et al, 2011, MNRAS, 415, 1479

Whitaker K. E., van Dokkum P. G., Brammer G., Franx M., 2012, ApJ, 754, L29

Wright A. H., Driver S. P., Robotham A. S. G., 2018, MNRAS, 480, 3491

Yang C., González-Alfonso E., Omont A., Pereira-Santaella M., Fischer J., Beelen A., Gavazzi R., 2020, A\&A, 634, L3

Yang C., Omont A., Beelen A., Gao Y., van der Werf P., Gavazzi R., Zhang Z. Y., Ivison R. et al, 2017, A\&A, 608, A144

Yun M. S., Scott K. S., Guo Y., Aretxaga I., Giavalisco M., Austermann J. E., Capak P., Chen Y. et al, 2012, MNRAS, 420,957

\section{APPENDIX A: OPTICAL, NIR AND MIR COUNTERPARTS FOR BAND-6 AND BAND-4 SELECTED GALAXIES}

Fig. 5 showed the expected $u, g, r, J, H, K, 4.5 \mu \mathrm{m}, 12 \mu \mathrm{m}$ and $22 \mu \mathrm{m}$ apparent magnitudes for band- 7 selected sources as a function of redshift. Here we show the equivalent for band-6 (Fig. A1) and band-4 (Fig. A2) selected sources. We remind the reader that due to the wavelength range of the IR templates of Dale et al. (2014) used in this work, the observer-frame band-4 emission is only well defined at $z \geq$ 0.84. At fixed flux and redshift, galaxies selected in band-4 are brighter than those in band- 6 and band- 7 .

Fig. A3 shows the variations in the $\mathrm{CO}(1-0)$ brightness luminosity in the SFR-stellar mass plane in the redshift window $1 \leq z \leq 4$. Analysis of this figure is presented in $\S$ refCOmodel.

\section{APPENDIX B: SPLINE FITS OF THE SFR AND $\mathrm{H}_{2}$ DENSITY REDSHIFT EVOLUTION OF SMGS}

We fit the evolution of the CSFRD and $\rho_{\mathrm{H}_{2}}$ of Figs. 12 and 13 of all the flux selected samples with a spline function and present the parameters in Table B1. These can be compared to observational estimates as ALMA surveys become public.

\section{APPENDIX C: INTRINSIC PROPERTIES OF GALAXIES IN THE $(\mathrm{U}-\mathrm{R})$ VS. $(\mathrm{R}-\mathrm{J})$ COLOUR PLANE}

Table C1 shows the median stellar mass, sSFR, distance to the MS and dust-to-stellar mass ratio of galaxies with $S_{\text {band-7 }}>1$ mJy that fall inside/outside the passive region shown in Fig. 6. We show this for 3 redshift bins, from $z \approx 2$ to $z \approx 4$. SHARK predicts that the typical star-forming galaxies that fall in the passive region of the $(\mathrm{u}-\mathrm{r})$ vs. $(\mathrm{r}-\mathrm{J})$ colour plane (and therefore could be considered contaminants) are higher stellar mass, higher dustto-stellar mass ratio, but lower sSFR than galaxies that fall in the SF region of the colour-colour plane, at fixed redshift. The latter tend to be above main sequence, while the former tend to be below. However, given the typical 

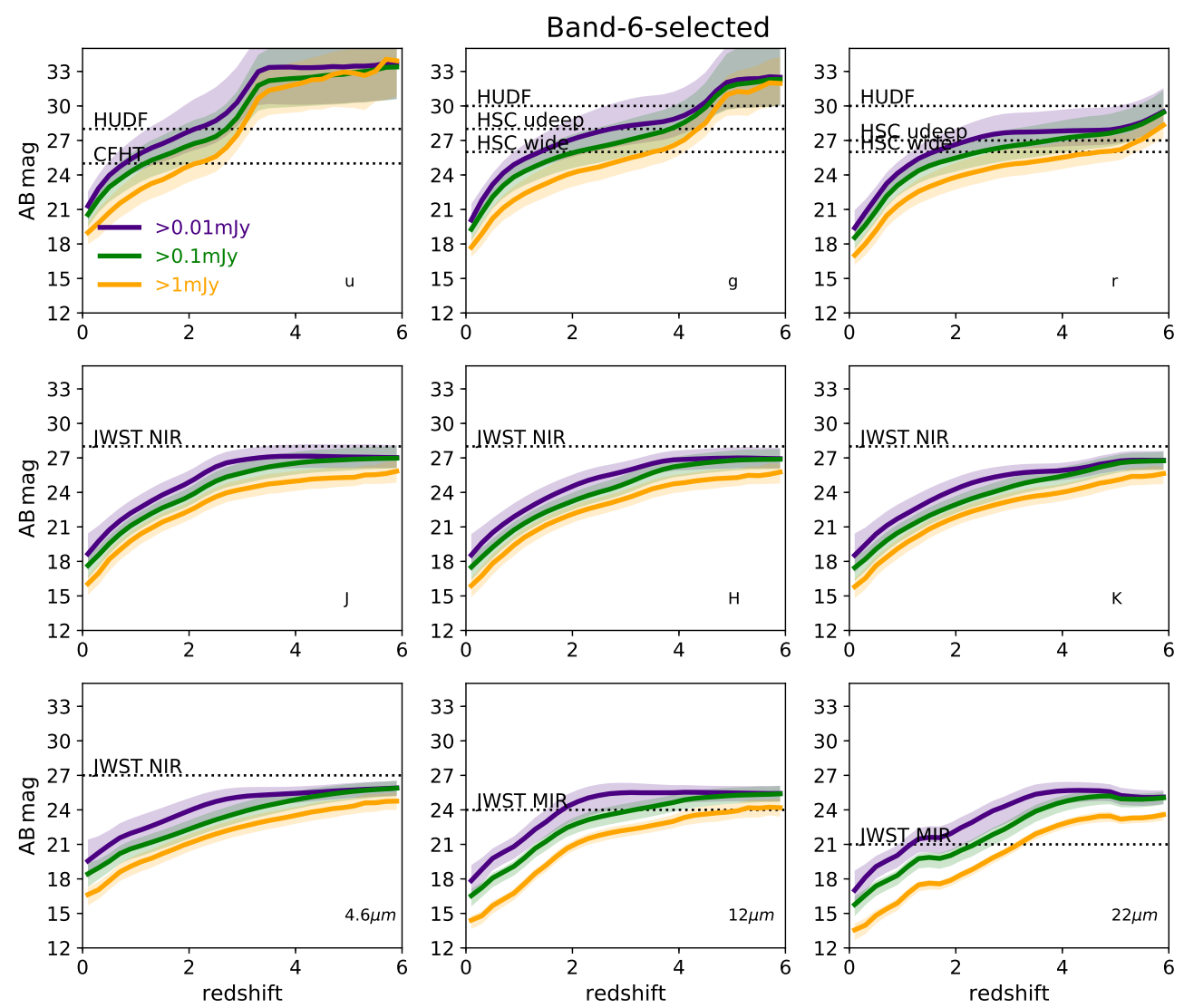

Figure A1. As Fig. 5 but for ALMA band- 6 selected galaxies.

criterion to classify galaxies as "main sequence" $\left(\Delta_{\mathrm{MS}}=\right.$ $\log _{10}\left(\mathrm{sSFR} / \mathrm{sSFR}_{\mathrm{MS}}\right)=[-0.6,0.6]$; Béthermin et al. 2015$)$, these galaxies would all be considered as such. The trends here are qualitatively the same when we analyse instead galaxies with $S_{\text {band }-7}>0.1 \mathrm{mJy}$.

This paper has been typeset from a $\mathrm{T}_{\mathrm{E}} \mathrm{X} / \mathrm{LAT}_{\mathrm{E}} \mathrm{X}$ file prepared by the author. 

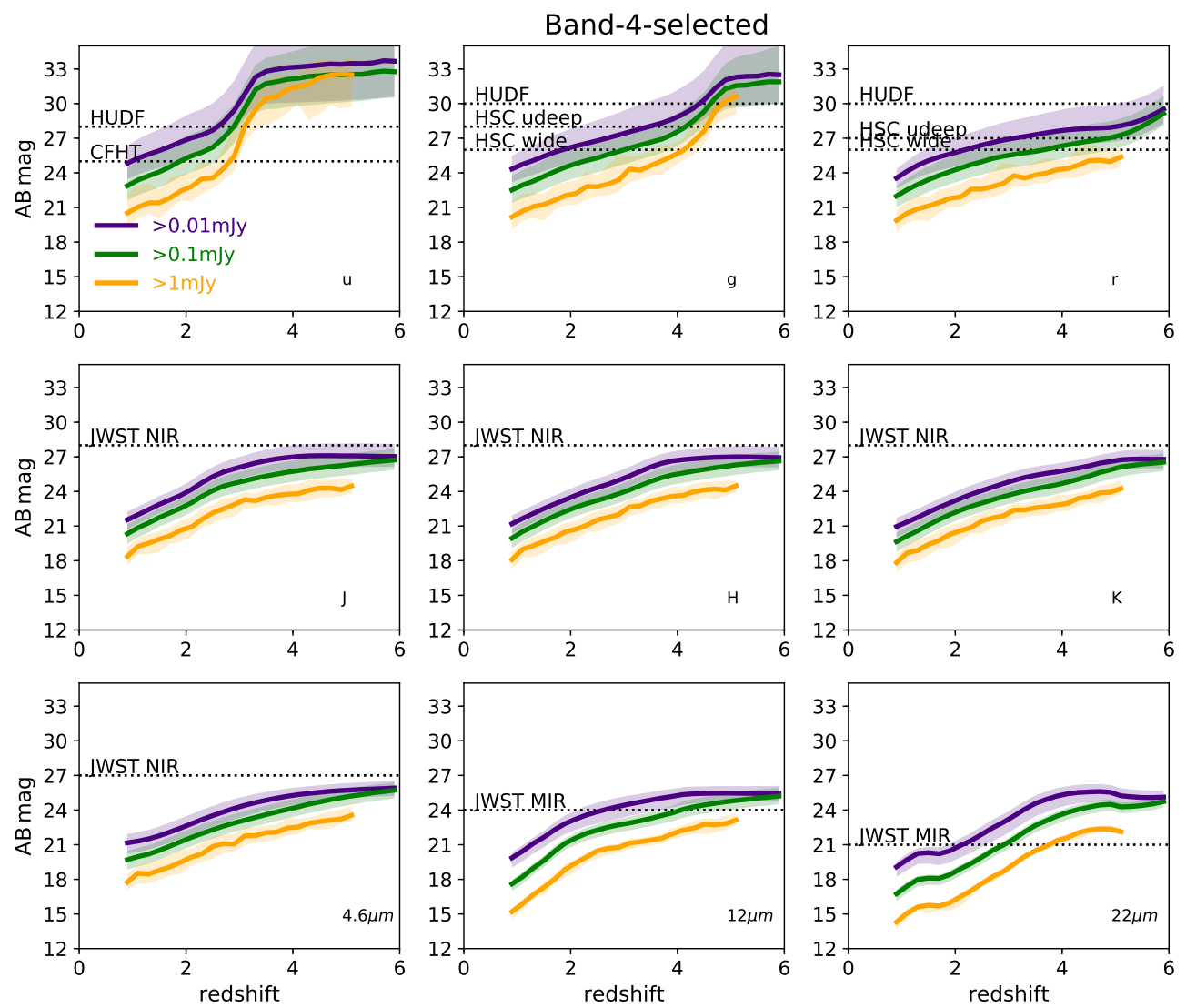

Figure A2. As Fig. 5 but for ALMA band-4 selected galaxies.
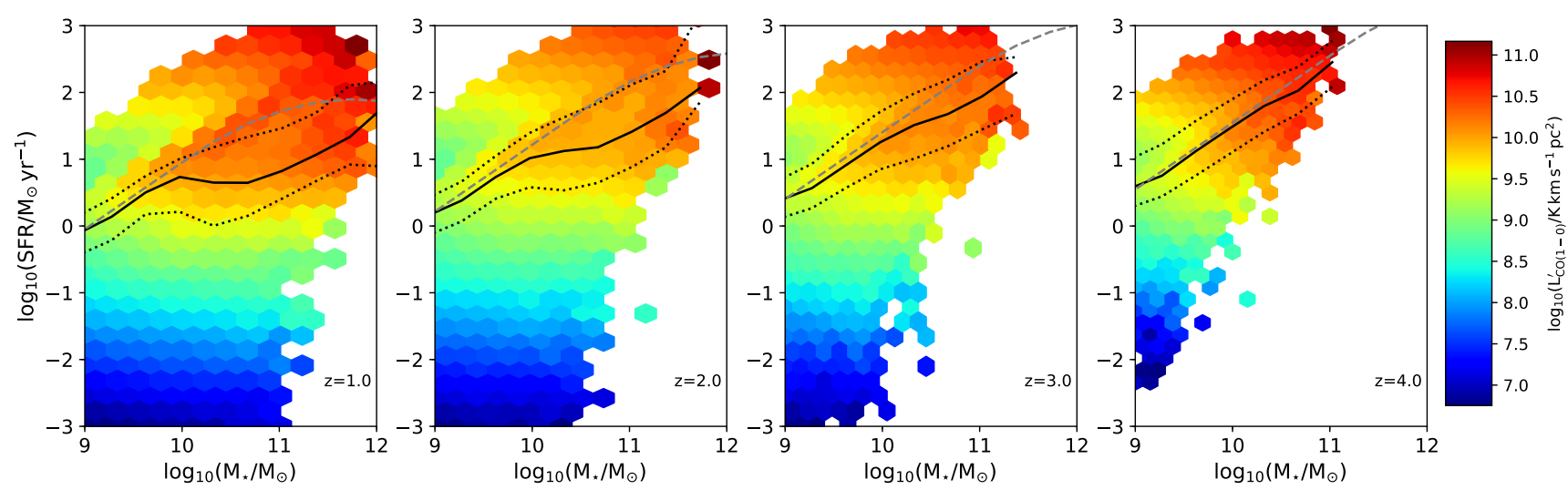

Figure A3. SFR vs. stellar masses plane at four different redshifts from $z=1$ to $z=4$, as labelled, using a $\Delta z=0.15$. Pixels with $\geq 10$ galaxies are coloured by their median $\mathrm{CO}(1-0)$ brightness luminosity. Solid and dotted lines show the median SFR and $16^{\text {th }}-84^{\text {th }}$ percentile ranges in bins of stellar mass, respectively, of all galaxies with SFR $>0$. For reference, we also show the MS inferred observationally by Schreiber et al. (2015) as dashed lines. 
Table B1. Best spline fit parameters in Figs. 12 and 13 for the 4 different flux samples in three ALMA bands 7, 6 and 4. The equations fitted are $\log _{10}\left(\rho_{\mathrm{SFR}}\right) \mid \log _{10}\left(\rho_{\mathrm{H}_{2}}\right)=a_{0}+a_{1} z+a_{2} z^{2}+$ $a_{3} z^{3}$, with $\rho_{\mathrm{SFR}}$ and $\rho_{\mathrm{H}_{2}}$ being in units of $\mathrm{M}_{\odot} \mathrm{yr}^{-1} \mathrm{cMpc}^{-3}$ and $\mathrm{M}_{\odot} \mathrm{cMpc}^{-3}$, respectively.

\begin{tabular}{|c|c|c|c|c|}
\hline Sample & $a_{0}$ & $a_{1}$ & $a_{2}$ & $a_{3}$ \\
\hline SFR & Evolution & & & \\
\hline \multicolumn{5}{|l|}{ band 7} \\
\hline$S<0.01 \mathrm{mJy}$ & -2.93 & 0.4 & -0.06 & 0.0015 \\
\hline $0.01<S<0.1 \mathrm{mJy}$ & -2.76 & 0.38 & -0.06 & 0.0014 \\
\hline $0.1<S<1 \mathrm{mJy}$ & -2.1 & 0.29 & -0.06 & 0.0002 \\
\hline$S>1 \mathrm{mJy}$ & -2.1 & 0.46 & -0.12 & $-10^{-7}$ \\
\hline \multicolumn{5}{|l|}{ band 6} \\
\hline$S<0.01 \mathrm{mJy}$ & -2.76 & 0.36 & -0.06 & 0.001 \\
\hline $0.01<S<0.1 \mathrm{mJy}$ & -2.4 & 0.25 & -0.05 & 0.001 \\
\hline $0.1<S<1 \mathrm{mJy}$ & -1.95 & 0.23 & -0.05 & -0.0003 \\
\hline$S>1 \mathrm{mJy}$ & -2.38 & 0.49 & -0.1 & -0.001 \\
\hline \multicolumn{5}{|l|}{ band 4} \\
\hline$S<0.01 \mathrm{mJy}$ & -1.9 & 0.14 & -0.003 & $4 \times 10^{-4}$ \\
\hline $0.01<S<0.1 \mathrm{mJy}$ & -1.76 & 0.09 & -0.003 & $6 \times 10^{-4}$ \\
\hline $0.1<S<1 \mathrm{mJy}$ & -2.6 & 0.56 & -0.12 & 0.004 \\
\hline$S>1 \mathrm{mJy}$ & -3.17 & 0.19 & -0.1 & 0.006 \\
\hline $\mathrm{H}_{2}$ & Evolution & & & \\
\hline \multicolumn{5}{|l|}{ band 7} \\
\hline$S<0.01 \mathrm{mJy}$ & 6.1 & 0.4 & -0.05 & $8.5 \times 10^{-4}$ \\
\hline $0.01<S<0.1 \mathrm{mJy}$ & 6.13 & 0.5 & -0.1 & $4.4 \times 10^{-3}$ \\
\hline $0.1<S<1 \mathrm{mJy}$ & 6.93 & 0.2 & -0.09 & $2.8 \times 10^{-3}$ \\
\hline$S>1 \mathrm{mJy}$ & 6.71 & 0.1 & -0.06 & $-3 \times 10^{-3}$ \\
\hline \multicolumn{5}{|l|}{ band 6} \\
\hline$S<0.01 \mathrm{mJy}$ & 6.23 & 0.34 & -0.05 & $8 \times 10^{-4}$ \\
\hline $0.01<S<0.1 \mathrm{mJy}$ & 6.5 & 0.34 & -0.09 & 0.003 \\
\hline $0.1<S<1 \mathrm{mJy}$ & 7 & 0.1 & -0.06 & 0.001 \\
\hline$S>1 \mathrm{mJy}$ & 6.2 & 0.16 & -0.04 & -0.006 \\
\hline \multicolumn{5}{|l|}{ band 4} \\
\hline$S<0.01 \mathrm{mJy}$ & 7.03 & 0.096 & -0.02 & $-2 \times 10^{-4}$ \\
\hline $0.01<S<0.1 \mathrm{mJy}$ & 7.12 & 0.002 & -0.04 & 0.001 \\
\hline $0.1<S<1 \mathrm{mJy}$ & 5.7 & 0.5 & -0.1 & 0.004 \\
\hline$S>1 \mathrm{mJy}$ & 4.8 & 0.3 & -0.15 & 0.01 \\
\hline
\end{tabular}

Table C1. Median stellar mass, sSFR, $\Delta_{\mathrm{MS}}$ and dust-to-stellar mass ratio of galaxies with $S_{\text {band-7 }}>1 \mathrm{mJy}$ that fall inside (passive) or outside (SF) the passive region shown in Fig. 6 in the $(\mathrm{u}-\mathrm{r})$ vs. $(\mathrm{r}-\mathrm{J})$ colour plane at 3 different redshifts. Stellar masses and sSFR have units of $\mathrm{M}_{\odot}$ and $\mathrm{Gyr}^{-1}$, respectively.

\begin{tabular}{ccccc}
\hline Property & $\log _{10}\left(\mathrm{M}_{\star}\right)$ & $\log _{10}(\mathrm{sSFR})$ & $\Delta_{\mathrm{MS}}$ & $\log _{10}\left(\mathrm{M}_{\mathrm{dust}} / \mathrm{M}_{\star}\right)$ \\
\hline$z=2 \pm 0.5$ & & & & \\
\hline passive & 11 & -0.62 & -0.6 & -2.38 \\
$\mathrm{SF}$ & 10.4 & 0.6 & 0.6 & -2.52 \\
\hline \hline$z=3 \pm 0.5$ & & & & \\
\hline passive & 10.7 & -0.11 & -0.28 & -2.39 \\
SF & 10.2 & 0.79 & 0.59 & -2.44 \\
\hline \hline$z=4 \pm 0.5$ & & & & -2.38 \\
\hline passive & 10.3 & 0.56 & 0.22 & -2.43 \\
SF & 10.1 & 0.95 & 0.54 & \\
\hline
\end{tabular}

